\title{
Anomaly-corrected supersymmetry algebra and supersymmetric holographic renormalization
}

\author{
Ok Song $\mathrm{An}^{1}$ \\ Department of Mathematics, SISSA, Via Bonomea 265, \\ 34136 Trieste, Italy \\ E-mail: oan@ictp.it
}

ABSTRACT: We present a systematic approach to supersymmetric holographic renormalization for a generic $5 \mathrm{D} \mathcal{N}=2$ gauged supergravity theory with matter multiplets, including its fermionic sector, with all gauge fields consistently set to zero. We determine the complete set of supersymmetric local boundary counterterms, including the finite counterterms that parameterize the choice of supersymmetric renormalization scheme. This allows us to derive holographically the superconformal Ward identities of a 4D superconformal field theory on a generic background, including the Weyl and super-Weyl anomalies. Moreover, we show that these anomalies satisfy the Wess-Zumino consistency condition. The superWeyl anomaly implies that the fermionic operators of the dual field theory, such as the supercurrent, do not transform as tensors under rigid supersymmetry on backgrounds that admit a conformal Killing spinor, and their anticommutator with the conserved supercharge contains anomalous terms. This property is explicitly checked for a toy model. Finally, using the anomalous transformation of the supercurrent, we obtain the anomaly-corrected supersymmetry algebra on curved backgrounds admitting a conformal Killing spinor.

Keywords: AdS-CFT Correspondence, Anomalies in Field and String Theories, Gaugegravity correspondence

ARXIV EPRINT: 1703.09607

\footnotetext{
${ }^{1}$ Now at: Department of Physics, Kim Il Sung University, RyongNam Dong, TaeSong District, Pyongyang, DPR. Korea.
} 


\section{Contents}

1 Introduction 1

$2 \mathcal{N}=2$ gauged SUGRA action in 5D 5

3 Radial Hamiltonian dynamics $\quad 6$

3.1 Radial Hamiltonian 6

3.2 Hamilton-Jacobi equations and the holographic renormalization 11

$\begin{array}{lll}3.3 & \text { Flow equations and leading asymptotics } & 13\end{array}$

4 Solution of the Hamilton-Jacobi equation $\quad \mathbf{1 4}$

$\begin{array}{lll}4.1 \text { Bosonic sector } & 15\end{array}$

$\begin{array}{lll}4.2 & \text { Fermionic sector } & 17\end{array}$

$\begin{array}{lll}4.3 & \text { Logarithmically divergent terms in 4D } & 20\end{array}$

4.4 Generic finite counterterms in 4D and summary 21

$\begin{array}{ll}4.5 & \text { Application to a toy model } \\ \end{array}$

5 Holographic dictionary and Ward identities 23

5.1 Ward identities and anomalies 24

$\begin{array}{ll}5.2 & \text { SUSY transformation of operators }\end{array}$

$\begin{array}{lll}5.3 & \text { BPS relations } & 30\end{array}$

5.4 Conserved charges and supersymmetry algebra with anomaly correction 33

$6 \quad$ Neumann boundary conditions $\quad 36$

$\begin{array}{lll}7 & \text { Concluding remarks } & 37\end{array}$

A Notation, conventions for Gamma matrices and useful identities $\quad 38$

B ADM decomposition and generalized PBH transformation 40

B.1 ADM decomposition of vielbein and the strong Fefferman-Graham gauge $\quad 40$

B.2 Decomposition of the covariant derivatives 42

B.3 Equations of motion and leading asymptotics of fermionic fields 42

B.4 Generalized PBH transformations 44

C Decomposition of the action and the fermion boundary terms 46

C.1 Decomposition of the kinetic action of the hyperino field 46

$\begin{array}{ll}\text { C.2 Gravitino part } & 47\end{array}$

$\begin{array}{lll}\text { C.3 Decomposition of the other terms } & 47\end{array}$

D Variation of the canonical momenta under the generalized PBH trans$\begin{array}{ll}\text { formation } & 48\end{array}$

E Derivation of the SUSY algebra without using Poisson bracket 48 


\section{Introduction}

Supersymmetric (SUSY) field theories in curved backgrounds [1-3] (see also [4] for a recent review) have received much attention in recent years, since they provide a playground where physically interesting, non-perturbative, results can often be obtained through localization techniques $[5,6]$.

Formulating consistent SUSY field theories in curved space usually consists of two steps [1]; the first one is to find the classical supergravity theory (SUGRA) by coupling a flat-space supersymmetric (SUSY) field theory to the gravity multiplet, and the second one is to take a rigid limit of SUGRA such that the gravity multiplet becomes non-dynamical, but maintains a non-trivial background value. Consistency requires that there exists at least one SUSY transformation of the SUGRA under which this background gravity multiplet should be invariant, namely

$$
\delta_{\eta} e_{(0) i}^{a}=0, \quad \delta_{\eta} \Psi_{(0)+i}=0, \quad \cdots,
$$

where $e_{(0) i}^{a}$ refers to the vielbein and $\Psi_{(0)+i}$ is the gravitino field and $\eta$ refers to the spinor parameter of the preserved SUSY. We refer to appendix A and B for notations and conventions. The requirement that the variation of the bosonic fields vanish is trivially satisfied on bosonic backgrounds.

One then derives the SUSY transformation of the local operators and the SUSY algebra in curved space from the corresponding ones of SUGRA. However, they are classical in the sense that the SUSY transformation laws and algebra derived in this way do not reflect any quantum effects.

To clarify this point, let us schematically discuss these quantum effects for a theory with an $\mathcal{N}=14 \mathrm{D}$ superconformal field theory (SCFT) as a UV fixed point. For this aim, we derive the Ward identities which contain UV data of quantum field theories. These Ward identities can be obtained in a local renormalization group language [7] without relying on a classical Lagrangian description, see e.g. section 2.3 in [8] for a recent review. In $\mathcal{N}=1$ SCFT, we have two local fermionic transformations, supersymmetry and superWeyl, respectively

$$
\begin{array}{lll}
\delta_{\epsilon_{+}} e_{(0) i}^{a}=-\frac{1}{2} \bar{\Psi}_{(0)+i} \Gamma^{a} \epsilon_{+}, & \delta_{\epsilon_{+}} \Psi_{(0)+i}=\mathbb{D}_{i} \epsilon_{+}+\cdots, & \cdots \\
\delta_{\epsilon_{-}} e_{(0) i}^{a}=0, & \delta_{\epsilon_{-}} \Psi_{(0)+i}=-\widehat{\Gamma}_{i} \epsilon_{-}+\cdots, & \cdots
\end{array}
$$

where the ellipses indicate possible contributions from other fields in the gravity multiplet and higer-order terms in fermions. Requiring the generating functional of connected correlation functions, $W\left[g_{(0) i j}, \Psi_{(0)+i}, \cdots\right]$, to be invariant under these local transformations up to a possible anomaly, we obtain two local operator equations, namely

$$
\begin{aligned}
\frac{1}{2} \mathcal{T}_{a}^{i} \bar{\Psi}_{(0)+i} \Gamma^{a}-\overline{\mathcal{S}}^{i} \overleftarrow{\mathbb{D}}_{i}+\cdots & =\overline{\mathcal{A}}_{s}, \\
-\overline{\mathcal{S}}^{i} \widehat{\Gamma}_{(0) i}+\cdots & =\overline{\mathcal{A}}_{\mathrm{sW}}
\end{aligned}
$$

where $\mathcal{T}_{a}^{i}$ and $\mathcal{S}^{i}$ refer to the energy-momentum tensor and supercurrent operator, respectively. Note that the Ward identities hold for generic backgrounds, even those where the 
fermionic sources are turned on. Combining these two Ward identities with the parameters $\eta_{+}$and $\eta_{-}$, which satisfy conformal Killing spinor (CKS) condition

$$
\delta_{\eta} \Psi_{(0)+i} \equiv \delta_{\eta_{+}} \Psi_{(0)+i}+\delta_{\eta_{-}} \Psi_{(0)+i}=\mathbb{D}_{i} \eta_{+}-\widehat{\Gamma}_{i} \eta_{-}=0,
$$

to the lowest order in fermions, we obtain the SUSY- $\eta$ Ward identity

$$
-\frac{1}{2} \mathcal{T}_{a}^{i} \bar{\Psi}_{(0)+i} \Gamma^{a} \eta_{+}+D_{i}\left(\overline{\mathcal{S}}^{i} \eta_{+}\right)+\cdots=-\left(\overline{\mathcal{A}}_{s} \eta_{+}+\overline{\mathcal{A}}_{\mathrm{sW}} \eta_{-}\right) \equiv \mathcal{A}_{\eta}
$$

where the fermionic sources are still turned on, because the CKS equation (1.4) to the lowest order in fermions does not require the background to be bosonic. One can see from the operator equation (1.5) that the SUSY- $\eta$ anomaly $\mathcal{A}_{\eta}$ should depend on the fermionic background sources, such as the gravitino field $\Psi_{+i}$. Therefore, one may not notice the existence of $\mathcal{A}_{\eta}$ on a bosonic background.

Ward identities such as (1.5) turn out to be rather useful. ${ }^{1}$ For instance, they determine the variation of quantum operators under the corresponding symmetry transformations, see e.g. (2.3.7) in [9]. It then follows from (1.5) that on (bosonic) supersymmetric backgrounds the supercurrent operator $\mathcal{S}^{i}$ transforms under the SUSY- $\eta$ transformation as

$$
\left.\delta_{\eta} \mathcal{S}^{i}\right|_{\text {susy-backgrounds }}=\left(-\frac{1}{2} \mathcal{T}_{a}^{i} \Gamma^{a} \eta_{+}-\frac{\delta}{\delta \bar{\Psi}_{(0)+i}} \mathcal{A}_{\eta}+\cdots\right)_{\text {susy-backgrounds }} .
$$

We emphasize that the anomalous term $\frac{\delta}{\delta \bar{\Psi}_{(0)+i}} \mathcal{A}_{\eta}$ does not appear in the 'classical' SUSY variation of the supercurrent operator $\mathcal{S}^{i}$, and it is non-zero in generic curved backgrounds admitting a conformal Killing spinor. Moreover, by integrating (1.6) over a Cauchy surface, one can obtain the commutator of two supercharges (see e.g. (2.6.14) and (2.6.15) in [9]) and find that it is also corrected by the anomalous term.

The upshot is that once the Ward identities (1.3) are found, one can see immediately all these quantum corrections. The main obstacle in obtaining (1.3) is to find out the anomalies $\mathcal{A}_{s}$ and $\mathcal{A}_{\mathrm{sW}}$. Fortunately, we have a nice tool for computing the anomalies, namely the AdS/CFT correspondence [10-12]. The holographic computation of the quantum anomalies, such as the computation of the Weyl anomaly in [13], results in specific values for the anomaly coefficients. For instance, one gets $a=c$ Weyl anomaly from a holographic calculation of two-derivative supergravity in $\mathrm{AdS}_{5}$. To obtain the whole class of anomalies one should consider a higher-derivative action. We emphasize that since the anomalies belonging to the same multiplet are related by SUSY transformations, the superWeyl anomaly $\mathcal{A}_{\mathrm{sW}}$ obtained by a holographic computation also has specific values for the anomaly coefficients.

Henceforth, in order to obtain the Ward identities of $4 \mathrm{D} \mathcal{N}=1$ SCFT by AdS/CFT, we consider a generic $\mathcal{N}=25 \mathrm{D}$ gauged SUGRA, including its fermionic sector, in asymptotically locally AdS (AlAdS) spaces, particular examples of which were studied in [14-19]. ${ }^{2}$

\footnotetext{
${ }^{1}$ One should keep in mind that the conservation law which allows to construct the conserved supercharge with non-covariantly-constant rigid parameter $\eta_{+}$is $D_{i}\left(\overline{\mathcal{S}}^{i} \eta_{+}\right)=0$, not $\overline{\mathcal{S}}^{i} \overleftarrow{\mathbb{D}}_{i} \eta_{+}=0$.

${ }^{2}$ Even though the solution considered in [19] is not AlAdS, the general form of the action given there is the same with the one here.
} 
More specifically, the SUGRA theory we consider is specified by a scalar superpotential $\mathcal{W}$ and its field content consists of a vielbein, two gravitini, as well as an equal number of spin-1/2 and scalar fields with negative mass-squared in order for the space to be asymptotically AdS. All gauge fields are consistently set to zero for simplicity. We study this theory up to quadratic order in the fermions. Having a stable AlAdS solution requires that $\mathcal{W}$ has an isolated local extremum. We also demand that $\mathcal{W}$ is a analytic function around that point.

As indicated in $[17,20]$, the $\mathcal{N}=25 \mathrm{D}$ gauged SUGRA can have a scalar superpotential $\mathcal{W}$ in several cases. A typical case is when there are only vector multiplets and a $\mathrm{U}(1)_{R}$ (subgroup of $\mathrm{SU}(2)_{R}$ R-symmetry group) is gauged [21]. When there are also hypermultiplets, the gauged SUGRA can have a scalar superpotential under a certain constraint related to the 'very special geometry' on the scalar manifold of the vector multiplets, which we do not discuss here in detail.

As in field theory, renormalization is required also in the bulk holographic computation. Although it has been studied since the early period of the AdS/CFT correspondence, most works on holographic renormalization (HR) [13, 22-31] have focused on the bosonic sector. $[18,19,32-37]$ obtained some boundary counterterms for the fermionic sector, but typically these were limited to either lower dimensional spacetime (mainly 3 or 4 dimensions) or to homogeneous solutions which do not depend on the transverse directions. We note that in a context different from this paper, $4 \mathrm{D} \mathcal{N}=1$ SUGRA including the fermionic sector was treated in [38] by a somehow ad hoc approach.

In this paper we perform HR following the approach of $[23,28,30,39]$. By formulating the theory in radial Hamiltonian language, we obtain the radial Hamiltonian, which gives the first class constraints. From the Hamiltonian constraint we obtain the HamiltonJacobi (HJ) equation, enabling us to determine the divergent counterterms in a covariant way without relying on a specific solution of the classical SUGRA. We emphasize that the counterterms, as the solution of HJ equation, should also satisfy the rest of the first class constraints. General covariance of the counterterms is a necessary and sufficient condition to satisfy diffeomorphism constraint, which is one of the first class constraints.

Once the counterterms are obtained, one can renormalize the canonical momenta of the radial Hamiltonian and thus obtain the renormalized canonical momenta. According to the AdS/CFT dictionary, the renormalized canonical momenta correspond to local operators of the field theory in the local renormalization group language [7]. The first class constraints turn out to be relations between local sources and operators, from which we obtain the Ward identities (see (5.2)) that in fact reflect the symmetries of the dual field theory and do not rely on a Lagrangian description of the quantum field theory. Since the bulk theory is a $5 \mathrm{D} \mathcal{N}=2$ SUGRA, the dual field theory has $4 \mathrm{D} \mathcal{N}=1$ superconformal symmetry and we obtain the corresponding Ward identities. Note that here we cannot see the $\mathrm{U}(1)_{R}$ symmetry because we truncate all gauge fields. In the related work [40] the $\mathrm{U}(1)_{R}$ gauge field is included in the model.

It turns out that the $\mathcal{N}=1$ superconformal symmetry is broken by anomalies. From the bulk point of view, these anomalies are due to the fact that some of the first class constraints are non-linear functions of the canonical momenta, implying that the corre- 
sponding symmetries are broken by the radial cut-off. From the dual field theory point of view, of course, the global anomalies are a quantum effect. We obtain not only the SUSYcompletion of the trace-anomaly, but also the holographic super-Weyl anomaly, ${ }^{3}$ which are rather interesting by themselves, since they can provide another tool for testing the AdS/CFT correspondence. ${ }^{4}$ As discussed before, we find that due to the anomaly, certain operators do not transform as tensors under super-Weyl transformation and the variation of operators gets an anomalous contribution, see (5.22). Hence, the $Q$-transformation of the operators also becomes anomalous, since it is obtained by putting together supersymmetry and super-Weyl transformations. Here $Q$ refers to the preserved supercharge. This is rather remarkable, since it implies that the 'classical' SUSY variation cannot become a total derivative in the path integral of SUSY field theories in curved space, unless the anomaly effects disappear. In this regard, it is shown in [40] that the 'new' non-covariant finite counterterms suggested in $[44,45]$ should be discarded since they were introduced in order to match with field theory without taking into account the anomaly-effect. From the anomalous transformation of the supercurrent operator, we find that the supersymmetry algebra in curved space is corrected by anomalous terms, see (5.56).

We finally note that the boundary conditions consistent with SUSY should be specified before the main computation of HR. In this work we always impose Dirichlet boundary conditions for the metric and the gravitino. As we will see, consistency with SUSY requires that either Dirichlet or Neumann boundary conditions should be imposed for scalars and their SUSY-partner spin 1/2 fields, together at the same time.

The rest of this paper is organized as follows. In section 2 we review the generic $\mathcal{N}=25$ D gauged SUGRA action and SUSY variation of the fields. In section 3, we first present the radial Hamiltonian and other first class constraints. We then systematically carry out the procedure of holographic renormalization and obtain the flow equations. In section 4 we determine the divergent counterterms and the possible finite counterterms. In particular, the complete set of counterterms is obtained explicitly for a toy model. By means of these counterterms, in section 5 we obtain the holographic Ward identities and anomalies and show that the anomalies satisfy the Wess-Zumino consistency condition. We then define constraint functions on the phase space of local sources and operators using the Ward identities, and we show that the symmetry transformation of the sources and operators are simply described in terms of the Poisson bracket with the corresponding constraint functions. Finally, we present the anomaly-corrected supersymmetry algebra on supersymmetric backgrounds. In section 6 we show that consistency with SUSY requires that scalars and their SUSY-partner fields should satisfy the same boundary condition. In appendix A, we describe our notations and present some useful identities. In appendix B

\footnotetext{
${ }^{3}$ Notice that the existence of a super-Weyl anomaly is natural, due to the existence of a Weyl anomaly that is related to the super-Weyl anomaly by a SUSY transformation.

${ }^{4}$ As we will see in the main text, our result for the super-Weyl anomaly is different from [41], which was obtained through a field theory calculation using Feynman diagrams. In [42], they tried to obtain the holographic super-Weyl anomaly, but their work is incomplete since contribution from the Ricci curvature is missed. In any case, we show that our result satisfies the Wess-Zumino (WZ) consistency conditions. One can check that the result of [41] does not satisfy the consistency conditions. See [43] for a review of WZ consistency conditions.
} 
we carry out some preliminary steps necessary in order to obtain the radial Hamiltonian, including the ADM decomposition, the strong Fefferman-Graham (FG) gauge, and the generalized Penrose-Brown-Henneaux (gPBH) transformations. In appendix C, we present the ADM decomposition of the radial Lagrangian part by part and in appendix $\mathrm{D}$ we prove that the $\mathrm{gPBH}$ transformations of the operators can be obtained from the holographic Ward identities. Finally, in appendix E we derive the anomaly-corrected SUSY algebra in an alternative way.

\section{$2 \mathcal{N}=2$ gauged SUGRA action in $5 \mathrm{D}$}

The action of gauged (on-shell) $(D=d+1=5)$ SUGRA admitting a scalar superpotential, with all gauge fields consistently truncated, is given by [19]

$$
S=S_{b}+S_{f}
$$

where

$$
\begin{aligned}
S_{b}=\frac{1}{2 \kappa^{2}} \int_{\mathcal{M}} d^{d+1} x \sqrt{-g}\left(R[g]-\mathcal{G}_{I J}(\varphi) \partial_{\mu} \varphi^{I} \partial^{\mu} \varphi^{J}-\mathcal{V}(\varphi)\right), \\
S_{f}=-\frac{1}{2 \kappa^{2}} \int_{\mathcal{M}} d^{d+1} x \sqrt{-g}\left\{\left(\bar{\Psi}_{\mu} \Gamma^{\mu \nu \rho} \nabla_{\nu} \Psi_{\rho}-\bar{\Psi}_{\mu} \overleftarrow{\nabla}_{\nu} \Gamma^{\mu \nu \rho} \Psi_{\rho}-\mathcal{W} \bar{\Psi}_{\mu} \Gamma^{\mu \nu} \Psi_{\nu}\right)\right. \\
\quad+\left(i \mathcal{G}_{I J} \bar{\zeta}^{I} \Gamma^{\mu}\left(\not \partial \varphi^{J}-\mathcal{G}^{J K} \partial_{K} \mathcal{W}\right) \Psi_{\mu}-i \mathcal{G}_{I J} \bar{\Psi}_{\mu}\left(\not \partial \varphi^{I}+\mathcal{G}^{I K} \partial_{K} \mathcal{W}\right) \Gamma^{\mu} \zeta^{J}\right) \\
\quad+\left(\mathcal{G}_{I J} \bar{\zeta}^{I}\left(\delta_{K}^{J} \not \nabla+\Gamma_{K L}^{J}[\mathcal{G}] \not \partial \varphi^{L}\right) \zeta^{K}-\mathcal{G}_{I J}\left[\bar{\zeta}^{I} \overleftarrow{\chi}^{J}+\bar{\zeta}^{K}\left(\not \partial \varphi^{L}\right) \Gamma_{K L}^{J} \zeta^{I}\right]\right) \\
\left.\quad+2 \mathcal{M}_{I J}(\varphi) \bar{\zeta}^{I} \zeta^{J}+\text { quartic terms }\right\},
\end{aligned}
$$

and the scalar potential and the mass matrix $\mathcal{M}_{I J}$ are expressed in terms of the superpotential as

$$
\begin{aligned}
\mathcal{V}(\varphi) & =\mathcal{G}^{I J} \partial_{I} \mathcal{W}(\varphi) \partial_{J} \mathcal{W}(\varphi)-\frac{d}{d-1} \mathcal{W}(\varphi)^{2}, \\
\mathcal{M}_{I J}(\varphi) & =\partial_{I} \partial_{J} \mathcal{W}-\Gamma_{I J}^{K}[\mathcal{G}] \partial_{K} \mathcal{W}-\frac{1}{2} \mathcal{G}_{I J} \mathcal{W} .
\end{aligned}
$$

Here $\kappa^{2}$ is related to the gravitational constant by $\kappa^{2}=8 \pi G_{(d+1)}$. Note that near the conformal boundary of AlAdS spaces (with radius 1), which we are interested in, the scalar potential and the superpotential take respectively the form

$$
\mathcal{V}(\varphi)=-d(d-1)+\mathcal{O}\left(\varphi^{2}\right), \quad \mathcal{W}(\varphi)=-(d-1)+\mathcal{O}\left(\varphi^{2}\right)
$$

The action (2.1) is, up to boundary terms, invariant under the supersymmetry transformation $^{5}$

$$
\begin{aligned}
\delta_{\epsilon} \varphi^{I} & =\frac{i}{2} \bar{\epsilon} \zeta^{I}+\text { h.c. }=\frac{i}{2}\left(\bar{\epsilon} \zeta^{I}-\bar{\zeta}^{I} \epsilon\right), \\
\delta_{\epsilon} E_{\mu}^{\alpha} & =\frac{1}{2} \bar{\epsilon} \Gamma^{\alpha} \Psi_{\mu}+\text { h.c. }=\frac{1}{2}\left(\bar{\epsilon} \Gamma^{\alpha} \Psi_{\mu}-\bar{\Psi}_{\mu} \Gamma^{\alpha} \epsilon\right),
\end{aligned}
$$

\footnotetext{
${ }^{5}$ In [19] the transformation rule of the gravitino field is given by $\delta_{\epsilon} \Psi_{\mu}=\left(\nabla_{\mu}+\frac{1}{6} \mathcal{W} \Gamma_{\mu}\right) \epsilon$, which is obtained by setting $D=5$ explicitly in $(2.8)$.
} 
where h.c. refers to hermitian conjugation, and

$$
\begin{aligned}
\delta_{\epsilon} \zeta^{I} & =-\frac{i}{2}\left(\not \partial \varphi^{I}-\mathcal{G}^{I J} \partial_{J} \mathcal{W}\right) \epsilon \\
\delta_{\epsilon} \Psi_{\mu} & =\left(\nabla_{\mu}+\frac{1}{2(d-1)} \mathcal{W} \Gamma_{\mu}\right) \epsilon .
\end{aligned}
$$

for any value of $d$.

Two comments are in order about the action (2.1). Firstly, all the fermions here, including the supersymmetry transformation parameter $\epsilon$, are Dirac fermions. In fact, in $\mathcal{N}=2$ five-dimensional SUGRA, the gravitino field is expressed in terms of a symplectic Majorana spinor [46], which can also be described in terms of a Dirac fermion [16]. Other fermions in the theory can also be expressed in the same way. Secondly, we would like to be as general as possible and thus, we keep $d$ generic in most of the following computations.

\section{Radial Hamiltonian dynamics}

According to the holographic dictionary [12] the on-shell action of the supergravity theory is the generating functional of the dual field theory. Therefore, the first step of the holographic computation is usually to consider the on-shell action on the bulk side. As is well-known, this on-shell action always suffers from long-distance divergences, which corresponds to the UV divergences of the dual field theory. Therefore, we need to renormalize the on-shell action of the supergravity theory, through holographic renormalization [13, 22-31].

The Hamiltonian formulation of holographic renormalization [23, 28, 30, 39] is arguably the most efficient, and as we will see, it helps make the analysis of the fermions tractable. The Hamiltonian constraint, one of the first class constraints obtained from the radial Hamiltonian, gives the Hamilton-Jacobi (HJ) equation by which we can obtain all the infinite counterterms for generic sources and curved background. Holographic renormalization essentially consists in determining all divergent terms in the on-shell action for generic background and sources in covariant form and subtracting them. Depending on the problem under consideration one can add some extra finite counterterms which actually correspond to the choice of renormalization scheme in the boundary field theory.

In this section we obtain the radial Hamiltonian, from which we extract the first class constraints. Afterwards, we present a general algorithm for obtaining the full counterterms from the HJ equation. We then obtain the flow equations which are needed to form a complete set of equations of motion.

\subsection{Radial Hamiltonian}

The Gibbons-Hawking term [47]

$$
\frac{1}{\kappa^{2}} \int_{\partial \mathcal{M}} d^{d} x \sqrt{-\gamma} K
$$

where $K$ is the extrinsic curvature on the boundary $\partial \mathcal{M}$, was introduced to have a welldefined variational problem for the Einstein-Hilbert action

$$
S_{E H}=\frac{1}{2 \kappa^{2}} \int_{\mathcal{M}} d^{d+1} x \sqrt{-g} R .
$$


As indicated in $[18,32-34,36]$, for the same reason some additional boundary terms are needed when the theory involves fermionic fields. For the action (2.1) these fermionic boundary terms turn out to be (for details, see appendix C.1 and C.2)

$$
\begin{aligned}
& \pm \frac{1}{2 \kappa^{2}} \int_{\partial \mathcal{M}} d^{d} x \sqrt{-\gamma} \bar{\Psi}_{i} \widehat{\Gamma}^{i j} \Psi_{j}, \\
& \pm \frac{1}{2 \kappa^{2}} \int_{\partial \mathcal{M}} d^{d} x \sqrt{-\gamma} \mathcal{G}_{I J} \bar{\zeta}^{I} \zeta^{J},
\end{aligned}
$$

where the signs in front of the terms bilinear in fermionic fields fixes which radiality (see (B.10)) of the fermion should be used as a generalized coordinate. Note, however, that the sign depends on the mass of the fermions and the choice of boundary conditions [34]. Since the mass of the gravitino $\Psi_{\mu}$ is $(d-1) / 2>0$, the sign of (3.3a) should be positive (see also appendix B.3 and B.4). The sign of the mass of $\zeta^{I}$ changes according to the model, and thus we cannot choose the sign of (3.3b) a priori.

For the time being, however, let us pick the + sign. As we will discuss in section 6 , picking the - sign corresponds to imposing Neumann boundary conditions on the spin- $1 / 2$ field $\zeta^{I}$. We emphasize that this choice of sign will not affect our claim later about the determination of the scalar fields' leading asymptotics. The whole action including the terms (3.1) and (3.3) is then given by

$$
S_{\text {full }}=S+\frac{1}{2 \kappa^{2}} \int_{\partial \mathcal{M}} d^{d} \mathrm{x} \sqrt{-\gamma}\left(2 K+\bar{\Psi}_{i} \widehat{\Gamma}^{i j} \Psi_{j}+\mathcal{G}_{I J} \bar{\zeta}^{I} \zeta^{J}\right)
$$

The full action $S_{\text {full }}$ can be written as $S_{\text {full }}=\int d r L$, where the radial Lagrangian $L$ is

$$
\begin{aligned}
L= & \frac{1}{2 \kappa^{2}} \int_{\Sigma_{r}} d^{d} x N \sqrt{-\gamma}\left\{R[\gamma]-\mathcal{G}_{I J} \partial_{i} \varphi^{I} \partial^{i} \varphi^{J}-\mathcal{V}(\varphi)+\left(\gamma^{i j} \gamma^{k l}-\gamma^{i k} \gamma^{j l}\right) K_{i j} K_{k l}\right. \\
& -\frac{\mathcal{G}_{I J}}{N^{2}}\left(\dot{\varphi}^{I}-N^{i} \partial_{i} \varphi^{I}\right)\left(\dot{\varphi}^{J}-N^{j} \partial_{j} \varphi^{J}\right)+\frac{2}{N}\left(\dot{\bar{\Psi}}_{+i} \widehat{\Gamma}^{i j} \Psi_{-j}+\bar{\Psi}_{-i} \widehat{\Gamma}^{i j} \dot{\Psi}_{+j}\right) \\
& +\frac{1}{N} \dot{e}_{a}^{i} e_{b}^{j}\left(\bar{\Psi}_{i} \Gamma^{a b} \Psi_{j}+\bar{\Psi}_{j} \Gamma^{b a} \Psi_{i}\right)+\left(K+\frac{1}{N} D_{k} N^{k}\right) \bar{\Psi}_{i} \widehat{\Gamma}^{i j} \Psi_{j}+\frac{1}{4 N} e_{a k} \dot{e}_{b}^{k} \bar{\Psi}_{i} \Gamma\left\{\widehat{\Gamma}^{i j}, \Gamma^{a b}\right\} \Psi_{j} \\
& +\frac{1}{2 N} K_{k l}\left[\left(\bar{\Psi}_{r}-N^{i} \bar{\Psi}_{i}\right)\left[\widehat{\Gamma}^{k j}, \widehat{\Gamma}^{l}\right] \Psi_{j}-\bar{\Psi}_{j}\left[\widehat{\Gamma}^{k j}, \widehat{\Gamma}^{l}\right]\left(\Psi_{r}-N^{i} \Psi_{i}\right)\right] \\
& +\frac{1}{4 N} \bar{\Psi}_{i}\left(2 \partial_{k} N\left[\widehat{\Gamma}^{i j}, \widehat{\Gamma}^{k}\right]-\left(D_{k} N_{l}\right) \Gamma\left\{\widehat{\Gamma}^{i j}, \widehat{\Gamma}^{k l}\right\}\right) \Psi_{j} \\
& -\frac{N^{i}}{N}\left(\bar{\Psi}_{j} \Gamma \widehat{\Gamma}^{j k} \mathbb{D}_{i} \Psi_{k}-\bar{\Psi}_{j} \overleftarrow{\mathbb{D}}_{i} \Gamma \widehat{\Gamma}^{j k} \Psi_{k}\right)-\bar{\Psi}_{i} \widehat{\Gamma}^{i j k} \mathbb{D}_{j} \Psi_{k}+\bar{\Psi}_{i} \overleftarrow{\mathbb{D}}_{j} \widehat{\Gamma}^{i j k} \Psi_{k} \\
& -\frac{1}{N} \bar{\Psi}_{k} \overleftarrow{\mathbb{D}}_{j} \Gamma \widehat{\Gamma}^{j k}\left(\Psi_{r}-N^{i} \Psi_{i}\right)-\frac{1}{N}\left(\bar{\Psi}_{r}-N^{i} \bar{\Psi}_{i}\right) \Gamma \widehat{\Gamma}^{j k} \mathbb{D}_{j} \Psi_{k} \\
& +\frac{1}{N} \bar{\Psi}_{k} \Gamma \widehat{\Gamma}^{j k}\left(\mathbb{D}_{j} \Psi_{r}-N^{i} \mathbb{D}_{j} \Psi_{i}\right)+\frac{1}{N}\left(\bar{\Psi}_{r} \overleftarrow{\mathbb{D}}_{j}-N^{i} \bar{\Psi}_{i} \overleftarrow{\mathbb{D}}_{j}\right) \Gamma \widehat{\Gamma}^{j k} \Psi_{k} \\
& +\frac{1}{N} \mathcal{W}\left[\left(\bar{\Psi}_{r}-N^{i} \bar{\Psi}_{i}\right) \widehat{\Gamma}^{j} \Psi_{j}+\bar{\Psi}_{j} \widehat{\Gamma}^{j} \Gamma\left(\Psi_{r}-N^{i} \Psi_{i}\right)\right]+\mathcal{W} \bar{\Psi}_{i} \widehat{\Gamma}^{i j} \Psi_{j} \\
& +\frac{2}{N} \mathcal{G}_{I J}\left(\bar{\zeta}_{+}^{I} \dot{\zeta}_{-}^{J}+\dot{\bar{\zeta}}_{-}^{I} \zeta_{+}^{J}\right)+\left(K+\frac{1}{N} D_{k} N^{k}\right) \mathcal{G}_{I J} \bar{\zeta}^{I} \zeta^{J}-\frac{1}{2 N} \mathcal{G}_{I J} e_{a i} \dot{e}_{b}^{i} \bar{\zeta}^{I} \Gamma^{a b} \Gamma \zeta^{J} \\
& +\frac{1}{N}\left(\dot{\varphi}^{K}-N^{i} \partial_{i} \varphi^{K}+N^{i} \partial_{i} \varphi^{K}\right) \partial_{K} \mathcal{G}_{I J} \bar{\zeta}^{I} \zeta^{J}-\mathcal{G}_{I J}\left(\bar{\zeta}^{I} \widehat{\Gamma}^{i} \mathbb{D}_{i} \zeta^{J}-\bar{\zeta}^{I} \overleftarrow{\mathbb{D}}_{i} \widehat{\Gamma}^{i} \zeta^{J}\right)
\end{aligned}
$$




$$
\begin{aligned}
& -\frac{1}{N} \mathcal{G}_{I J}\left[-\frac{1}{2} D_{i} N_{j}\left(\bar{\zeta}^{I} \widehat{\Gamma}^{i j} \Gamma \zeta^{J}\right)-N^{i} \bar{\zeta}^{I} \Gamma \mathbb{D}_{i} \zeta^{J}+N^{i}\left(\bar{\zeta}^{I} \overleftarrow{\mathbb{D}}_{i}\right) \Gamma \zeta^{J}\right] \\
& -\frac{i}{N} \mathcal{G}_{I J}\left[\frac{1}{N}\left(\dot{\varphi}^{J}-N^{j} \partial_{j} \varphi^{J}\right)\left[\bar{\zeta}^{I}\left(\Psi_{r}-N^{i} \Psi_{i}+N \widehat{\Gamma}^{i} \Gamma \Psi_{i}\right)-\left(\bar{\Psi}_{r}-N^{i} \bar{\Psi}_{i}+N \bar{\Psi}_{i} \Gamma \widehat{\Gamma}^{i}\right) \zeta^{I}\right]\right. \\
& \left.\quad+\partial_{i} \varphi^{J}\left[\bar{\zeta}^{I} \Gamma \widehat{\Gamma}^{i}\left(\Psi_{r}-N^{j} \Psi_{j}\right)-\left(\bar{\Psi}_{r}-N^{j} \bar{\Psi}_{j}\right) \widehat{\Gamma}^{i} \Gamma \zeta^{I}\right]+N \partial_{i} \varphi^{J}\left(\bar{\zeta}^{I} \widehat{\Gamma}^{j} \widehat{\Gamma}^{i} \Psi_{j}-\bar{\Psi}_{j} \widehat{\Gamma}^{i} \widehat{\Gamma}^{j} \zeta^{I}\right)\right] \\
& +\frac{i}{N} \partial_{I} \mathcal{W}\left[\bar{\zeta}^{I} \Gamma\left(\Psi_{r}-N^{i} \Psi_{i}\right)+\left(\bar{\Psi}_{r}-N^{i} \bar{\Psi}_{i}\right) \Gamma \zeta^{I}+N\left(\bar{\Psi}_{i} \widehat{\Gamma}^{i} \zeta^{I}+\bar{\zeta}^{I} \widehat{\Gamma}^{i} \Psi_{i}\right)\right] \\
& -\frac{1}{N} \partial_{K} \mathcal{G}_{I J}\left[\left(\dot{\varphi}^{J}-N^{i} \partial_{I} \varphi^{J}\right)\left(\bar{\zeta}^{I} \Gamma \zeta^{K}-\bar{\zeta}^{K} \Gamma \zeta^{I}\right)+N \partial_{i} \varphi^{J}\left(\bar{\zeta}^{I} \widehat{\Gamma}^{i} \zeta^{K}-\bar{\zeta}^{K} \widehat{\Gamma}^{i} \zeta^{I}\right)\right] \\
& \left.-2 \mathcal{M}_{I J} \bar{\zeta}^{I} \zeta^{J}\right\} .
\end{aligned}
$$

Given the radial Lagrangian $L$ we can derive the canonical momenta

$$
\begin{aligned}
& \pi_{a}^{i}=\frac{\delta L}{\delta \dot{e}_{i}^{a}}=\left(\delta_{j}^{i} e_{a k}+\delta_{k}^{i} e_{a j}\right) \frac{\sqrt{-\gamma}}{2 \kappa^{2}}\left[\left(\gamma^{j k} \gamma^{l m}-\gamma^{j l} \gamma^{k m}\right) K_{l m}+\frac{1}{2} \gamma^{j k}\left(\mathcal{G}_{I J} \bar{\zeta}^{I} \zeta^{J}+\bar{\Psi}_{p} \widehat{\Gamma}^{p q} \Psi_{q}\right)\right. \\
& \left.-\frac{1}{4 N}\left(\bar{\Psi}_{p}\left[\widehat{\Gamma}^{j p}, \widehat{\Gamma}^{k}\right]\left(\Psi_{r}-N^{l} \Psi_{l}\right)-\left(\bar{\Psi}_{r}-N^{l} \bar{\Psi}_{l}\right)\left[\widehat{\Gamma}^{j p}, \widehat{\Gamma}^{k}\right] \Psi_{p}\right)\right] \\
& -\frac{\sqrt{-\gamma}}{2 \kappa^{2}}\left[e^{b i}\left(\frac{1}{4} \bar{\Psi}_{j} \Gamma\left\{\widehat{\Gamma}^{j k}, \Gamma_{a b}\right\} \Psi_{k}-\frac{1}{2} \mathcal{G}_{I J} \bar{\zeta}^{I} \Gamma_{a b} \Gamma \zeta^{J}\right)+e_{a j}\left(\bar{\Psi}^{j} \widehat{\Gamma}^{i k} \Psi_{k}+\bar{\Psi}_{k} \widehat{\Gamma}^{k i} \Psi^{j}\right)\right], \\
& \pi_{I}^{\varphi}=\frac{\delta L}{\delta \dot{\varphi}^{I}}=\frac{\sqrt{-\gamma}}{2 N \kappa^{2}}\left[-2 \mathcal{G}_{I J}\left(\dot{\varphi}^{J}-N^{i} \partial_{i} \varphi^{J}\right)+N \partial_{I} \mathcal{G}_{J K} \bar{\zeta}^{J} \zeta^{K}-N \partial_{K} \mathcal{G}_{I J}\left(\bar{\zeta}^{J} \Gamma \zeta^{K}-\bar{\zeta}^{K} \Gamma \zeta^{J}\right)\right. \\
& \left.-i \mathcal{G}_{I J}\left(\bar{\zeta}^{J}\left(\Psi_{r}-N^{i} \Psi_{i}+N \widehat{\Gamma}^{i} \Gamma \Psi_{i}\right)-\left(\bar{\Psi}_{r}-N^{i} \bar{\Psi}_{i}+N \bar{\Psi}_{i} \Gamma \widehat{\Gamma}^{i}\right) \zeta^{J}\right)\right], \\
& \pi_{I}^{\zeta}=L \frac{\overleftarrow{\delta}}{\delta \dot{\zeta}_{-}^{I}}=\frac{\sqrt{-\gamma}}{\kappa^{2}} \mathcal{G}_{I J} \bar{\zeta}_{+}^{J} \\
& \pi_{I}^{\bar{\zeta}}=\frac{\vec{\delta}}{\delta \dot{\bar{\zeta}}_{-}^{I}} L=\frac{\sqrt{-\gamma}}{\kappa^{2}} \mathcal{G}_{I J} \zeta_{+}^{J}, \\
& \pi_{\Psi}^{i}=L \frac{\overleftarrow{\delta}}{\delta \dot{\Psi}_{+i}}=\frac{\sqrt{-\gamma}}{\kappa^{2}} \bar{\Psi}_{-j} \widehat{\Gamma}^{j i} \\
& \pi_{\bar{\Psi}}^{i}=\frac{\vec{\delta}}{\delta \dot{\bar{\Psi}}_{+i}} L=\frac{\sqrt{-\gamma}}{\kappa^{2}} \widehat{\Gamma}^{i j} \Psi_{-j}
\end{aligned}
$$

One should keep in mind that $\pi_{\bar{\Psi}}^{i}$ and $\pi_{\Psi}^{i}$ have negative radiality, and $\pi_{I}^{\zeta}$ and $\pi_{I}^{\zeta}$ have positive radiality.

From $K_{i j}=K_{j i}$, we obtain the constraint

$$
\begin{aligned}
0=\mathcal{J}_{a b} \equiv & \frac{\kappa^{2}}{\sqrt{-\gamma}}\left(e_{a}^{i} \pi_{b i}-e_{b}^{i} \pi_{a i}\right)-\frac{1}{4} \bar{\Psi}_{j} \Gamma\left\{\widehat{\Gamma}^{j k}, \Gamma_{a b}\right\} \Psi_{k}+\frac{1}{2} \mathcal{G}_{I J} \bar{\zeta}^{I} \Gamma_{a b} \Gamma \zeta^{J} \\
& -\frac{1}{2} e_{a}^{i} e_{b}^{j}\left(\bar{\Psi}_{i} \widehat{\Gamma}_{j k} \Psi^{k}+\bar{\Psi}^{k} \widehat{\Gamma}_{k j} \Psi_{i}-\bar{\Psi}_{j} \widehat{\Gamma}_{i k} \Psi^{k}-\bar{\Psi}^{k} \widehat{\Gamma}_{k i} \Psi_{j}\right),
\end{aligned}
$$


which, as we will see, corresponds to the local Lorentz generator of the frame bundle on the slice $\Sigma_{r}[36]$.

Inverting the canonical momenta ${ }^{6}$ and implementing the Legendre transform we obtain the radial Hamiltonian

$$
\begin{aligned}
H & =\int d^{d} x\left(\dot{e}_{i}^{a} \pi_{a}^{i}+\dot{\varphi}^{I} \pi_{I}^{\varphi}+\pi_{I}^{\zeta} \dot{\zeta}_{-}^{I}+\dot{\bar{\zeta}}_{-}^{I} \pi_{I}^{\zeta}+\pi_{\Psi}^{i} \dot{\Psi}_{+i}+\dot{\bar{\Psi}}_{+i} \pi_{\bar{\Psi}}^{i}\right)-L \\
& =\int d^{d} x\left[N \mathcal{H}+N_{i} \mathcal{H}^{i}+\left(\bar{\Psi}_{r}-N^{i} \bar{\Psi}_{i}\right) \mathcal{F}+\overline{\mathcal{F}}\left(\Psi_{r}-N^{i} \Psi_{i}\right)\right],
\end{aligned}
$$

where

$$
\begin{aligned}
& \mathcal{H}=\frac{\kappa^{2}}{2 \sqrt{-\gamma}}\left[\left(\frac{1}{d-1} e_{i}^{a} e_{j}^{b}-e_{j}^{a} e_{i}^{b}\right) \pi_{a}^{i} \pi_{b}^{j}-\mathcal{G}^{I J} \pi_{I}^{\varphi} \pi_{J}^{\varphi}+\mathcal{G}^{I J}\left(\pi_{I}^{\zeta \not D} \pi_{J}^{\bar{\zeta}}-\pi_{I}^{\zeta} \overleftarrow{\not D} \pi_{J}^{\bar{\zeta}}\right)\right. \\
& -\frac{1}{2(d-1)}\left(e^{a j} \pi_{a}^{i}+e^{a i} \pi_{a}^{j}\right)\left[(d-1)\left(\bar{\Psi}_{+i} \pi_{\bar{\Psi} j}+\pi_{\Psi j} \Psi_{+i}\right)+\pi_{\Psi}^{p}\left(\widehat{\Gamma}_{p i}-(d-2) \gamma_{p i}\right) \widehat{\Gamma}_{j}^{k} \Psi_{+k}\right. \\
& \left.+\bar{\Psi}_{+k} \widehat{\Gamma}^{k}{ }_{j}\left(\widehat{\Gamma}_{i p}-(d-2) \gamma_{i p}\right) \pi_{\bar{\Psi}}^{p}\right]+\frac{1}{d-1} e_{i}^{a} \pi_{a}^{i}\left(-\bar{\zeta}_{-}^{I} \pi_{I}^{\bar{\zeta}}-\pi_{I}^{\zeta} \zeta_{-}^{I}+\bar{\Psi}_{+j} \pi_{\Psi}^{j}+\pi_{\Psi}^{j} \Psi_{+j}\right) \\
& +2 \mathcal{G}^{I J} \Gamma_{J K}^{L}[\mathcal{G}] \pi_{I}^{\varphi}\left(\bar{\zeta}_{-}^{K} \pi_{L}^{\bar{\zeta}}+\pi_{L}^{\zeta} \zeta_{-}^{K}\right)+i \pi_{I}^{\varphi}\left[\frac{1}{d-1}\left(\bar{\zeta}_{-}^{I} \widehat{\Gamma}_{i} \pi_{\bar{\Psi}}^{i}+\pi_{\Psi}^{i} \widehat{\Gamma}_{i} \zeta_{-}^{I}\right)\right. \\
& \left.-\mathcal{G}^{I J}\left(\pi_{I}^{\zeta} \widehat{\Gamma}^{i} \Psi_{+i}+\bar{\Psi}_{+i} \widehat{\Gamma}^{i} \pi_{J}^{\bar{\zeta}}\right)\right]-\pi_{\Psi}^{k}\left[\left(\frac{1}{d-1} \widehat{\Gamma}_{k} \widehat{\Gamma}_{j}-\gamma_{k j}\right) \not D-\overleftarrow{\not D}\left(\frac{1}{d-1} \widehat{\Gamma}_{k} \widehat{\Gamma}_{j}-\gamma_{k j}\right)\right] \pi_{\bar{\Psi}}^{j} \\
& +\frac{i}{d-1}\left(\pi_{I}^{\zeta} \not \partial \varphi^{I} \widehat{\Gamma}_{i} \pi_{\bar{\Psi}}^{i}-\pi_{\Psi}^{i} \widehat{\Gamma}_{i} \not \partial \varphi^{I} \pi_{I}^{\bar{\zeta}}\right)-2 i \partial_{i} \varphi^{I}\left(\pi_{I}^{\zeta} \pi_{\bar{\Psi}}^{i}-\pi_{\Psi}^{i} \pi_{I}^{\bar{\zeta}}\right) \\
& \left.+\mathcal{G}^{I M} \mathcal{G}^{K N} \partial_{i} \varphi^{J}\left(\partial_{K} \mathcal{G}_{I J}-\partial_{I} \mathcal{G}_{K J}\right) \pi_{M}^{\zeta} \widehat{\Gamma}^{i} \pi_{N}^{\bar{\zeta}}\right] \\
& -\frac{1}{2} \mathcal{W}\left(\bar{\Psi}_{+i} \pi_{\bar{\Psi}}^{i}+\pi_{\Psi}^{i} \Psi_{+i}\right)+\mathcal{M}_{I J}\left(\mathcal{G}^{I K} \pi_{K}^{\zeta} \zeta_{-}^{J}+\mathcal{G}^{J K} \bar{\zeta}_{-}^{I} \pi_{K}^{\bar{\zeta}}\right) \\
& -\frac{i}{2} \partial_{I} \mathcal{W}\left[\mathcal{G}^{I J}\left(\bar{\Psi}_{+i} \widehat{\Gamma}^{i} \pi_{J}^{\bar{\zeta}}+\pi_{J}^{\zeta} \widehat{\Gamma}^{i} \Psi_{+i}\right)+\frac{1}{d-1}\left(\pi_{\Psi}^{i} \widehat{\Gamma}_{i} \zeta_{-}^{I}+\bar{\zeta}_{-}^{I} \widehat{\Gamma}_{i} \pi_{\Psi}^{i}\right)\right] \\
& +\frac{\sqrt{-\gamma}}{2 \kappa^{2}}\left[-R[\gamma]+\mathcal{G}_{I J} \partial_{i} \varphi^{I} \partial^{i} \varphi^{J}+\mathcal{V}(\varphi)+\mathcal{G}_{I J} \bar{\zeta}_{-}^{I}(\not \triangleright D-\overleftarrow{\not D}) \zeta_{-}^{J}+\bar{\Psi}_{+i} \widehat{\Gamma}^{i j k}\left(\mathbb{D}_{j}-\overleftarrow{\mathbb{D}}_{j}\right) \Psi_{+k}\right. \\
& +D_{k}\left(\bar{\Psi}_{+i}\left(\gamma^{j k} \widehat{\Gamma}^{i}-\gamma^{i k} \widehat{\Gamma}^{j}\right) \Psi_{+j}\right)+i \mathcal{G}_{I J} \partial_{i} \varphi^{J}\left(\bar{\zeta}_{-}^{I} \widehat{\Gamma}^{j} \widehat{\Gamma}^{i} \Psi_{+j}-\bar{\Psi}_{+j} \widehat{\Gamma}^{i} \widehat{\Gamma}^{j} \zeta_{-}^{I}\right) \\
& \left.+\partial_{K} \mathcal{G}_{I J} \partial_{i} \varphi^{J}\left(\bar{\zeta}_{-}^{I} \widehat{\Gamma}^{i} \zeta_{-}^{K}-\bar{\zeta}_{-}^{K} \widehat{\Gamma}^{i} \zeta_{-}^{I}\right)\right], \\
& \mathcal{H}^{i}=-e^{a i} D_{j} \pi_{a}^{j}+\left(\partial^{i} \varphi^{I}\right) \pi_{I}^{\varphi}+\left(\bar{\zeta}_{-}^{I} \overleftarrow{D}^{i}\right) \pi_{I}^{\bar{\zeta}}+\pi_{I}^{\zeta}\left(\mathbb{D}^{i} \zeta_{-}^{I}\right)+\pi_{\Psi}^{j}\left(\mathbb{D}^{i} \Psi_{+j}\right)+\left(\bar{\Psi}_{+j} \overleftarrow{\mathbb{D}}^{i}\right) \pi_{\bar{\Psi}}^{j} \\
& -D_{j}\left(\pi_{\Psi}^{j} \Psi_{+}^{i}+\bar{\Psi}_{+}^{i} \pi_{\bar{\Psi}}^{j}\right),
\end{aligned}
$$

${ }^{6}$ For instance, the inverse of the canonical momentum $\pi_{\Psi}^{i}$ is $\Psi_{-i}=\frac{\kappa^{2}}{\sqrt{-\gamma}} \frac{1}{d-1}\left[\widehat{\Gamma}_{i j}-(d-2) \gamma_{i j}\right] \pi_{\Psi}^{j}$. 


$$
\begin{aligned}
\mathcal{F}= & \frac{2 \kappa^{2}}{\sqrt{-\gamma}}\left\{\frac{1}{4(d-1)} \widehat{\Gamma}_{i} \pi_{\bar{\Psi}}^{i} e_{j}^{a} \pi_{a}^{j}-\frac{1}{8} \Gamma^{a} \gamma_{i k} \pi_{\Psi}^{k} \pi_{a}^{i}-\frac{1}{8} e_{l}^{a} \widehat{\Gamma}_{i} \pi_{\bar{\Psi}}^{l} \pi_{a}^{i}+\frac{i}{4} \mathcal{G}^{I J} \pi_{I}^{\varphi} \pi_{J}^{\bar{\zeta}}\right\} \\
& +\frac{1}{4} \Gamma^{a} \Psi_{+i} \pi_{a}^{i}+\frac{1}{4} \widehat{\Gamma}_{i} \Psi_{+j} e^{a j} \pi_{a}^{i}+\frac{i}{2} \pi_{I}^{\varphi} \zeta_{-}^{I}-\mathbb{D}_{i} \pi_{\bar{\Psi}}^{i}-\frac{1}{2(d-1)} \mathcal{W} \widehat{\Gamma}_{i} \pi_{\bar{\Psi}}^{i}-\frac{i}{2} \partial_{i} \varphi^{I} \widehat{\Gamma}^{i} \pi_{I}^{\bar{\zeta}} \\
& -\frac{i}{2} \mathcal{G}^{I J} \partial_{I} \mathcal{W} \pi_{J}^{\bar{\zeta}}+\frac{\sqrt{-\gamma}}{2 \kappa^{2}}\left(2 \widehat{\Gamma}^{i j} \mathbb{D}_{i} \Psi_{+j}+\mathcal{W} \widehat{\Gamma}^{i} \Psi_{+i}+i \mathcal{G}_{I J} \partial_{i} \varphi^{J} \widehat{\Gamma}^{i} \zeta_{-}^{I}+i\left(\partial_{I} \mathcal{W}\right) \zeta_{-}^{I}\right) .
\end{aligned}
$$

We note that in the above computations we used the local Lorentz constraint (3.7).

By radiality we split $\mathcal{F}$ into two parts

$$
\begin{aligned}
\mathcal{F}_{+} \equiv \Gamma_{+} \mathcal{F}= & \frac{\kappa^{2}}{2 \sqrt{-\gamma}}\left[\pi_{a}^{j} e^{a k}\left(\frac{1}{d-1} \gamma_{j k} \widehat{\Gamma}_{i}-\frac{1}{2} \gamma_{i j} \widehat{\Gamma}_{k}-\frac{1}{2} \gamma_{i k} \widehat{\Gamma}_{j}\right) \pi_{\Psi}^{i}+i \mathcal{G}^{I J} \pi_{I}^{\varphi} \pi_{J}^{\bar{\zeta}}\right] \\
& -\frac{1}{2(d-1)} \mathcal{W} \widehat{\Gamma}_{i} \pi_{\bar{\Psi}}^{i}-\frac{i}{2} \mathcal{G}^{I J} \partial_{I} \mathcal{W} \pi_{J}^{\bar{\zeta}}+\frac{\sqrt{-\gamma}}{2 \kappa^{2}}\left(2 \widehat{\Gamma}^{i j} \mathbb{D}_{i} \Psi_{+j}+i \mathcal{G}_{I J} \partial_{i} \varphi^{J} \widehat{\Gamma}^{i} \zeta_{-}^{I}\right),
\end{aligned}
$$

and

$$
\begin{aligned}
\mathcal{F}_{-} \equiv \Gamma_{-} \mathcal{F}= & \frac{1}{4}\left(\widehat{\Gamma}_{i} \Psi_{+j}+\widehat{\Gamma}_{j} \Psi_{+i}\right) e^{a j} \pi_{a}^{i}+\frac{i}{2} \pi_{I}^{\varphi} \zeta_{-}^{I} \\
& -\mathbb{D}_{i} \pi_{\Psi}^{i}-\frac{i}{2} \partial_{i} \varphi^{I} \widehat{\Gamma}^{i} \pi_{I}^{\bar{\zeta}}+\frac{\sqrt{-\gamma}}{2 \kappa^{2}}\left(\mathcal{W} \widehat{\Gamma}^{i} \Psi_{+i}+i \partial_{I} \mathcal{W} \zeta_{-}^{I}\right) .
\end{aligned}
$$

The canonical momenta for $N, N_{i}$ and $\Psi_{r}$ vanish identically, and it then follows from Hamilton's equations that

$$
\mathcal{H}=\mathcal{H}^{i}=\mathcal{F}_{-}=\mathcal{F}_{+}=0 .
$$

These first class constraints reflect respectively radial reparameterization invariance and diffeomorphism, supersymmetry and super-Weyl invariance along the radial slice $\Sigma_{r}$, which can be seen by comparing with (B.38).

Inserting (3.6) in (3.7), we obtain

$$
0=e_{a}^{i} \pi_{b i}-e_{b}^{i} \pi_{a i}+\frac{1}{2} \bar{\zeta}_{-}^{I} \Gamma_{a b} \pi_{I}^{\bar{\zeta}}-\frac{1}{2} \pi_{I}^{\zeta} \Gamma_{a b} \zeta_{-}^{I}-\frac{1}{2} \pi_{\Psi}^{i} \Gamma_{a b} \Psi_{+i}+\frac{1}{2} \bar{\Psi}_{+i} \Gamma_{a b} \pi_{\bar{\Psi}}^{i},
$$

which reflects the local frame rotation symmetry of the theory according to (B.38). We emphasize that at the bosonic level this local Lorentz constraint reduces to

$$
e_{a}^{i} \pi_{b i}=e_{b}^{i} \pi_{a i}
$$

which implies that we can define a symmetric canonical momentum for the metric through the relations

$$
\frac{\delta}{\delta \dot{\gamma}_{i j}} L^{B} \equiv \pi^{i j}=\frac{1}{2} e^{a j} \frac{\delta}{\delta \dot{e}_{i}^{a}} L^{B}
$$

Here $L^{B}$ denotes bosonic part of the radial Lagrangian (3.5).

We emphasize that the linearity of the constraints $\mathcal{H}^{i}=\mathcal{F}_{-}=0$ and the local Lorentz constraint reflect the fact that their corresponding symmetries are not broken by the radial cut-off. Meanwhile, the constraints $\mathcal{H}=0$ and $\mathcal{F}_{+}=0$ are quadratic in the momenta, implying that in fact the cut-off breaks these symmetries, though they are non-linearly realized in the bulk. 


\subsection{Hamilton-Jacobi equations and the holographic renormalization}

The HJ equations are obtained by inserting the expressions

$$
\pi_{a}^{i}=\frac{\delta}{\delta e_{i}^{a}} \mathbb{S}, \quad \pi_{I}^{\varphi}=\frac{\delta}{\delta \varphi^{I}} \mathbb{S}, \quad \pi_{I}^{\zeta}=\mathbb{S} \frac{\overleftarrow{\delta}}{\delta \zeta_{-}^{I}}, \quad \pi_{I}^{\bar{\zeta}}=\frac{\vec{\delta}}{\delta \bar{\zeta}_{-}^{I}} \mathbb{S}, \quad \pi_{\Psi}^{i}=\mathbb{S} \frac{\overleftarrow{\delta}}{\delta \Psi_{+i}}, \quad \pi_{\Psi}^{i}=\frac{\vec{\delta}}{\delta \bar{\Psi}_{+i}} \mathbb{S}
$$

for the canonical momenta in the first class constraints (3.14). Here $\mathbb{S}\left[e, \varphi, \zeta_{-}, \Psi_{+}\right]$is Hamilton's principal functional.

Hamilton's principal functional $\mathbb{S}$ is particularly important since it can be identified with the on-shell action evaluated with a radial cut-off $\Sigma_{r}$. Holographically renormalizing the on-shell action only requires solving these HJ equations for $\mathbb{S}$ up to the finite terms, without relying on the specific solution of the equations of motion. Since this asymptotic solution of the HJ equations is obtained in covariant form for generic sources, we can identify the divergent terms with the sought after boundary counterterms, which cancel the divergences of the on-shell action as well as of all correlation functions.

As pointed out in [48], the constraint $\mathcal{H}^{i}=0$ and the local Lorentz constraint (3.15) which reflects the bulk diffeomorphism invariance along the transverse direction is automatically satisfied as long as we look for a local and covariant solution. Hence, the equations we have to solve are the constraints $\mathcal{H}=\mathcal{F}_{-}=\mathcal{F}_{+}=0$.

Let us briefly review the algorithm of solving the HJ equation in AlAdS geometry. In general, the Hamiltonian constraint is solved asymptotically by using the formal expansion of $\mathbb{S}$ with respect to the dilatation operator $\delta_{D}[30]$ (see section 5.2 of [8] for a recent review)

$$
\delta_{D}=\int d^{d} x \sum_{\Phi}\left(\Delta_{\Phi}-d\right) \frac{\delta}{\delta \Phi}
$$

where $\Phi$ refers to every field in the theory and $\Delta_{\Phi}$ denotes the scaling dimension of the operator dual to $\Phi$. The solution takes form of

$$
\mathbb{S}=\int_{\Sigma_{r}} d^{d} x \sqrt{-\gamma} \mathcal{L}=\int_{\Sigma_{r}} d^{d} x \sqrt{-\gamma}\left(\mathcal{L}_{[0]}+\mathcal{L}_{[1]}+\cdots+\widetilde{\mathcal{L}}_{[d]} \log e^{-2 r}+\mathcal{L}_{[d]}+\cdots\right),
$$

where

$$
\delta_{D} \mathcal{L}_{[n]}=-n \mathcal{L}_{[n]}, 0 \leq n<d, \quad \delta_{D} \widetilde{\mathcal{L}}_{[d]}=-d \widetilde{\mathcal{L}}_{[d]} .
$$

Since the dilatation operator $\delta_{D}$ asymptotically coincides with the radial derivative

$$
\partial_{r}=\int_{\Sigma_{r}} d^{d} x \sum_{\Phi} \dot{\Phi} \frac{\delta}{\delta \Phi}
$$

in AlAdS, one can see that $\mathcal{L}_{[n]}$ for $n<d$ and $\widetilde{\mathcal{L}}_{[d]}$ are asymptotically divergent, and can therefore be identified with the boundary counterterms, namely

$$
\mathbb{S}_{\mathrm{ct}}=-\int_{\Sigma_{r}} d^{d} x \sqrt{-\gamma}\left(\mathcal{L}_{[0]}+\mathcal{L}_{[1]}+\cdots+\widetilde{\mathcal{L}}_{[d]} \log e^{-2 r}\right)
$$

By construction, this is the full set of all possible divergent terms. 
This general argument of finding $\mathbb{S}_{\mathrm{ct}}$ is not suitable in our case, since the operator $\delta_{D}$ requires knowledge of all scaling dimensions in the theory from the onset. Since we do not want to specify the scaling dimension of the scalars $\varphi^{I}$ and of the fermions $\zeta^{I}$ in advance, we will instead seek a solution for $\mathbb{S}$ in an expansion in eigenfunctions of the alternative operator

$$
\delta_{e}=\int d^{d} x\left(e_{i}^{a} \frac{\delta}{\delta e_{i}^{a}}+\frac{1}{2} \bar{\Psi}_{+i} \frac{\delta}{\delta \bar{\Psi}_{+i}}+\frac{1}{2} \frac{\overleftarrow{\delta}}{\delta \Psi_{+i}} \Psi_{+i}\right)
$$

rather than $\delta_{D}[39,48]$, since we know that the scaling dimension of the operators dual to $e_{i}^{a}$ and $\Psi_{+i}$ in AlAdS are $d+1$ and $d+1 / 2$ respectively, see appendix B.3. Note that $\delta_{e}$ basically counts powers of the vielbein and the gravitino. The formal expansion of Hamilton's principal function $\mathbb{S}\left[e, \varphi, \zeta_{-}, \Psi_{+}\right]$with respect to $\delta_{e}$ is thus

$$
\mathbb{S}=\mathbb{S}_{(0)}+\mathbb{S}_{(1)}+\mathbb{S}_{(2)}+\cdots, \quad \mathbb{S}_{(k)} \equiv \int d^{d} x \mathbb{L}_{(k)},
$$

where $\delta_{e} \mathbb{S}_{(k)}=(d-k) \mathbb{S}_{(k)}$. This implies that

$$
\pi_{(k) a}^{i} e_{i}^{a}+\frac{1}{2} \pi_{(k) \Psi}^{i} \Psi_{+i}+\frac{1}{2} \bar{\Psi}_{+i} \pi_{(k) \bar{\Psi}}^{i}=(d-k) \mathbb{L}_{(k)}+\partial_{i} v_{(k)}^{i},
$$

for certain $v_{(k)}^{i}$. However, the Lagrangian $\mathbb{L}_{(k)}$ is defined up to a total derivative, and thus we can put [39]

$$
\pi_{(k) a}^{i} e_{i}^{a}+\frac{1}{2} \pi_{(k) \Psi}^{i} \Psi_{+i}+\frac{1}{2} \bar{\Psi}_{+i} \pi_{(k) \bar{\Psi}}^{i}:=(d-k) \mathbb{L}_{(k)} .
$$

As we will see later, this identification of $\mathbb{L}_{(k)}$ greatly simplifies the HJ equation and makes it almost algebraic.

By using (3.27) we can solve the HJ equation recursively, but this procedure stops at $\mathbb{S}_{(d)}$, which has $\delta_{e}$ weight zero. The reason why higher-order terms, which are finite in $r \rightarrow \infty$ limit, cannot be determined in this recursive procedure is that they are related to the arbitrary integration constants which form a complete integral together with the integration constants from the flow equations, see [39] for explanation in more detail.

Assuming that the all scalar and spin-1/2 operators are not irrelevant, we find that any term with negative $\delta_{e}$ weight should have negative dilatation weight, see (3.19). This implies that all the divergent terms appear up to $\mathbb{S}_{(d)}$ so that we can identify the counterterms as

$$
S_{\mathrm{ct}}=-\sum_{k=0}^{2 d} \mathbb{S}_{(k / 2)}
$$

Note that the logarithmically divergent terms are distributed in almost all of the $\mathbb{S}_{(k)} \mathrm{s}$ with $0 \leq k \leq d$. Since our radial slice is four-dimensional, these terms appear with the pole $1 /(d-4)$. Converting this pole by (dimensional regularization) $[30,39]$

$$
\frac{1}{d-4} \rightarrow-\frac{1}{2} \log e^{-2 r}
$$


and summing up all of them, we obtain the logarithmically divergent terms $\widetilde{\mathcal{L}}_{[d]}$. We emphasize that the two algorithms we described in fact give the same result for $S_{\mathrm{ct}}$.

Once the local counterterms $S_{\mathrm{ct}}$ are obtained, we renormalize the on-shell action by

$$
\widehat{S}_{\mathrm{ren}}=\lim _{r \rightarrow+\infty}\left(S_{\mathrm{full}}+S_{\mathrm{ct}}\right)=\lim _{r \rightarrow+\infty} \int_{\Sigma_{r}} d^{d} x \mathcal{L}_{[d]} .
$$

The canonical momenta are automatically renormalized by $\mathbb{S}_{\mathrm{ct}}$, namely

$$
\widehat{\pi}^{\Phi} \equiv \pi^{\Phi}+\frac{\delta}{\delta \Phi} S_{\text {ct }}, \text { for every field } \Phi,
$$

and the variation of the renormalized on-shell action under any variation of fields is given by the chain rule

$$
\delta \widehat{S}_{\mathrm{ren}}=\lim _{r \rightarrow+\infty} \int d^{d} x\left(\widehat{\pi}_{a}^{i} \delta e_{i}^{a}+\widehat{\pi}_{I}^{\varphi} \delta \varphi^{I}+\delta \bar{\zeta}_{-}^{I} \widehat{\pi}_{I}^{\zeta}+\widehat{\pi}_{I}^{\zeta} \delta \zeta_{-}^{I}+\delta \bar{\Psi}_{+i} \widehat{\pi}_{\Psi}^{i}+\widehat{\pi}_{\Psi}^{i} \delta \Psi_{+i}\right)
$$

\subsection{Flow equations and leading asymptotics}

The flow equations are obtained by substituting (3.18) into Hamilton's equations

$$
\begin{aligned}
& \dot{e}_{i}^{a}=\frac{\delta H}{\delta \pi_{a}^{i}}, \quad \dot{\pi}_{a}^{i}=-\frac{\delta H}{\delta e_{i}^{a}}, \\
& \dot{\varphi}^{I}=\frac{\delta H}{\delta \pi_{I}^{\varphi}}, \quad \dot{\pi}_{I}^{\varphi}=-\frac{\delta H}{\delta \varphi^{I}}, \\
& \dot{\zeta}_{-}^{I}=\frac{\delta}{\delta \pi_{I}^{\zeta}} H, \quad \dot{\pi}_{I}^{\zeta}=-H \frac{\delta}{\delta \zeta_{-}}, \quad \dot{\bar{\zeta}}_{-}^{I}=H \frac{\delta}{\delta \pi_{I}^{\bar{\zeta}}}, \quad \dot{\pi}_{I}^{\bar{\zeta}}=-\frac{\delta}{\delta \bar{\zeta}_{-}^{I}} H \\
& \dot{\Psi}_{+i}=\frac{\delta}{\delta \pi_{\Psi}^{i}} H, \quad \dot{\pi}_{\Psi}^{i}=-H \frac{\delta}{\delta \Psi_{+i}}, \quad \dot{\bar{\Psi}}_{+i}=H \frac{\delta}{\delta \pi_{\bar{\Psi}}^{i}}, \quad \dot{\pi}_{\bar{\Psi}}^{i}=-\frac{\delta}{\delta \bar{\Psi}_{+i}} H .
\end{aligned}
$$

The resulting flow equations are

$$
\begin{aligned}
\dot{e}_{i}^{a}= & \frac{\kappa^{2}}{2 \sqrt{-\gamma}}\left\{2\left(\frac{1}{d-1} e_{i}^{a} e_{j}^{b}-e_{j}^{a} e_{i}^{b}\right) \pi_{b}^{j}-\frac{1}{2(d-1)} e^{a j}\left[(d-1)\left(\bar{\Psi}_{+i} \pi_{\bar{\Psi} j}+\pi_{\Psi j} \Psi_{+i}\right)\right.\right. \\
& \left.-\pi_{\Psi}^{p}\left[\widehat{\Gamma}_{p i}-(d-2) \gamma_{p i}\right] \widehat{\Gamma}_{j}^{k} \Psi_{+k}+\bar{\Psi}_{+k} \widehat{\Gamma}_{j}^{k}\left[\widehat{\Gamma}_{i p}-(d-2) \gamma_{i p}\right] \pi_{\bar{\Psi}}^{p}+(i \leftrightarrow j)\right] \\
& \left.+\frac{1}{d-1} e_{i}^{a}\left(-\bar{\zeta}_{-}^{I} \pi_{I}^{\bar{\zeta}}-\pi_{I}^{\zeta} \zeta_{-}^{I}+\bar{\Psi}_{+j} \pi_{\bar{\Psi}}^{j}+\pi_{\Psi}^{j} \Psi_{+j}\right)\right\} \\
\dot{\varphi}^{I}= & \frac{\kappa^{2}}{\sqrt{-\gamma}} \mathcal{G}^{I J}\left[-\pi_{J}^{\varphi}+\Gamma_{J L}^{K}[\mathcal{G}]\left(\pi_{K}^{\zeta} \zeta_{-}^{L}+\bar{\zeta}_{-}^{L} \pi_{K}^{\bar{\zeta}}\right)-\frac{i}{2}\left(\pi_{J}^{\zeta} \widehat{\Gamma}^{i} \Psi_{+i}+\bar{\Psi}_{+i} \widehat{\Gamma}^{i} \pi_{J}^{\bar{\zeta}}\right)\right] \\
& +\frac{\kappa^{2}}{\sqrt{-\gamma}} \frac{i}{2(d-1)}\left(\bar{\zeta}_{-}^{I} \widehat{\Gamma}_{i} \pi_{\Psi}^{i}+\pi_{\Psi}^{i} \widehat{\Gamma}_{i} \zeta_{-}^{I}\right),
\end{aligned}
$$




$$
\begin{aligned}
\dot{\Psi}_{+i}= & \frac{\kappa^{2}}{2 \sqrt{-\gamma}}\left[-\frac{1}{2}\left(\delta_{i}^{k} e^{a j}+\gamma^{j k} e_{i}^{a}\right) \pi_{a}^{k} \Psi_{+j}+\frac{1}{d-1} e_{j}^{a} \pi_{a}^{j} \Psi_{+i}+i \pi_{I}^{\varphi} \widehat{\Gamma}_{i} \zeta_{-}^{I}\right. \\
& -\frac{1}{2(d-1)}\left(e^{a j} \pi_{a}^{l}+e^{a l} \pi_{a}^{j}\right)\left(\widehat{\Gamma}_{i l}-(d-2) \gamma_{i l}\right) \widehat{\Gamma}_{j}^{k} \Psi_{+k}-\frac{i}{d-1} \widehat{\Gamma}_{i} \not \partial \varphi^{I} \pi_{J}^{\bar{\zeta}} \\
& \left.-\frac{2}{d-1}\left(\widehat{\Gamma}_{i j k}-(d-2) \gamma_{i j} \widehat{\Gamma}_{k}\right) \mathbb{D}^{k} \pi_{\bar{\Psi}}^{j}+2 i \partial_{i} \varphi^{I} \pi_{I}^{\bar{\zeta}}\right]-\frac{1}{2} \mathcal{W} \Psi_{+i} \\
& -\frac{i}{2(d-1)} \partial_{I} \mathcal{W} \widehat{\Gamma}_{i} \zeta_{-}^{I}
\end{aligned}
$$

and

$$
\begin{aligned}
\dot{\zeta}_{-}^{I}= & \frac{\kappa^{2}}{2 \sqrt{-\gamma}}\left[2 \mathcal{G}^{I J} \not \triangleright D \pi_{J}^{\bar{\zeta}}+\partial_{i} \mathcal{G}^{I J} \widehat{\Gamma}^{i} \pi_{J}^{\bar{\zeta}}-\frac{1}{d-1} e_{i}^{a} \pi_{a}^{i} \zeta_{-}^{I}+2 \mathcal{G}^{L J} \Gamma_{J K}^{I}[\mathcal{G}] \pi_{L}^{\varphi} \zeta_{-}^{K}\right. \\
& \left.\quad-i \mathcal{G}^{I J} \pi_{\varphi}^{J} \widehat{\Gamma}^{i} \Psi_{+i}-2 i \partial_{i} \varphi^{I} \pi_{\bar{\Psi}}^{i}+\mathcal{G}^{I M} \mathcal{G}^{K N} \partial_{i} \varphi^{J}\left(\partial_{K} \mathcal{G}_{J M}-\partial_{M} \mathcal{G}_{K J}\right) \widehat{\Gamma}^{i} \pi_{N}^{\bar{\zeta}}\right] \\
& +\mathcal{M}_{J K} \mathcal{G}^{I K} \zeta_{-}^{J}-\frac{i}{2} \partial^{I} \mathcal{W} \widehat{\Gamma}^{i} \Psi_{+i} .
\end{aligned}
$$

Here for simplicity we choose the gauge (B.13), which reduces the radial Hamiltonian $H$ to $H=\int d^{d} x \mathcal{H}$. We emphasize that the flow equations (3.34), (3.35), (3.36) and (3.37), together with the HJ equations, form a complete set of equations of motion of the theory. ${ }^{7}$

\section{Solution of the Hamilton-Jacobi equation}

To solve the HJ equation efficiently we divide Hamilton's principal function into several parts according to the structure of the various terms. Namely, we first split $\mathbb{S}$ into two sectors: $\mathbb{S}^{B}$, the purely bosonic part, and $\mathbb{S}^{F}$, which is quadratic in fermions. The terms in $\mathbb{S}^{F}$ are further split into three parts: $\mathbb{S} \zeta \zeta$ which contains quadratic terms in $\zeta_{-}^{I} \mathrm{~s}, \mathbb{S}^{\Psi \Psi}$ containing quadratic terms in $\Psi_{+i}$ and $\mathbb{S}^{\zeta \Psi}$, containing bilinears in $\zeta_{-}^{I}$ and $\Psi_{+i}$. In total,

$$
\mathbb{S}=\mathbb{S}^{B}+\mathbb{S}^{\zeta \zeta}+\mathbb{S}^{\Psi \Psi}+\mathbb{S}^{\zeta \Psi}
$$

Due to radiality and the Lorentz structure of the fermionic sources, the asymptotic expansion of $\mathbb{S}^{B}, \mathbb{S}^{\zeta \Psi}, \mathbb{S}^{\zeta \zeta}$ and $\mathbb{S}^{\Psi \Psi}$ should be

$$
\begin{aligned}
\mathbb{S}^{B} & =\mathbb{S}_{(0)}^{B}+\mathbb{S}_{(2)}^{B}+\mathbb{S}_{(4)}^{B}+\cdots, \\
\mathbb{S}^{\zeta \Psi} & =\mathbb{S}_{(3 / 2)}^{\zeta \Psi}+\mathbb{S}_{(7 / 2)}^{\zeta \Psi}+\cdots, \\
\mathbb{S}^{\zeta \zeta} & =\mathbb{S}_{(1)}^{\zeta \zeta}+\mathbb{S}_{(3)}^{\zeta \zeta}+\mathbb{S}_{(5)}^{\zeta \zeta}+\cdots, \\
\mathbb{S}^{\Psi \Psi} & =\mathbb{S}_{(2)}^{\Psi \Psi}+\mathbb{S}_{(4)}^{\Psi \Psi}+\cdots
\end{aligned}
$$

How to solve the HJ equation for the bosonic sector has been discussed in the literature [30, 39, 49], though it is difficult to solve the HJ equation for a completely general

\footnotetext{
${ }^{7}$ One can use the flow equations (3.36) and (3.37) to determine the asymptotic behavior of $\Psi_{+i}$ and $\zeta_{-}^{I}$, as is done in appendix B.3, instead of using the Euler-Lagrange equations (B.19) and (B.20).
} 
model. ${ }^{8}$ The key feature is that after finding the solution of the HJ equation to leading order, we only need to solve a (almost algebraic) first-order differential equation for the higher orders, thanks to the relation (3.27). Nevertheless, these first-order differential equations are not easy to solve at the first attempt.

Here we have another set of first-order differential equations, namely $\mathcal{F}_{-}=\mathcal{F}_{+}=0$. These are relatively simpler than the Hamiltonian constraint $\mathcal{H}=0$, so one can try to solve these constraints first. Not surprisingly, it works well, in particular for the fermionic sector, and the solution is totally consistent with the other constraints, as we will see soon.

\subsection{Bosonic sector}

Let us first consider the bosonic sector. The corresponding Hamiltonian constraint $\mathcal{H}=0$ is

$$
\begin{aligned}
\frac{\kappa^{2}}{2 \sqrt{-\gamma}}\left[4\left(\frac{1}{d-1} \gamma_{i j} \gamma_{k l}-\gamma_{i k} \gamma_{j l}\right)\right. & \left.\frac{\delta \mathbb{S}^{B}}{\delta \gamma_{i j}} \frac{\delta \mathbb{S}^{B}}{\delta \gamma_{k l}}-\mathcal{G}^{I J} \frac{\delta \mathbb{S}^{B}}{\delta \varphi^{I}} \frac{\delta \mathbb{S}^{B}}{\delta \varphi^{J}}\right] \\
+ & \frac{\sqrt{-\gamma}}{2 \kappa^{2}}\left(-R[\gamma]+\mathcal{G}_{I J} \partial_{i} \varphi^{I} \partial^{i} \varphi^{J}+\mathcal{V}(\varphi)\right)=0 .
\end{aligned}
$$

One can readily see that the HJ equation for $\mathbb{S}_{(0)}$ is

$$
\frac{\kappa^{2}}{2 \sqrt{-\gamma}}\left[4\left(\frac{1}{d-1} \gamma_{i j} \gamma_{k l}-\gamma_{i k} \gamma_{j l}\right) \frac{\delta \mathbb{S}_{(0)}}{\delta \gamma_{i j}} \frac{\delta \mathbb{S}_{(0)}}{\delta \gamma_{k l}}-\mathcal{G}^{I J} \frac{\delta \mathbb{S}_{(0)}}{\delta \varphi^{I}} \frac{\delta \mathbb{S}_{(0)}}{\delta \varphi^{J}}\right]+\frac{\sqrt{-\gamma}}{2 \kappa^{2}} \mathcal{V}(\varphi)=0
$$

The leading term of $\mathbb{S}, \mathbb{S}_{(0)}$, should not contain any derivatives and must be purely bosonic so that its ansatz becomes

$$
\mathbb{S}_{(0)}=-\frac{1}{\kappa^{2}} \int d^{d} x \sqrt{-\gamma} \mathrm{U}(\varphi) .
$$

Substituting this ansatz into the constraint $\mathcal{F}_{-}=0$, we obtain

$$
\frac{1}{4}\left(\widehat{\Gamma}_{i} \Psi_{+j}+\widehat{\Gamma}_{j} \Psi_{+i}\right) e^{a j} \frac{\delta \mathbb{S}_{(0)}}{\delta e_{i}^{a}}+\frac{\sqrt{-\gamma}}{2 \kappa^{2}} \mathcal{W} \widehat{\Gamma}_{i} \Psi_{+i}=0
$$

and find the unique solution for $\mathrm{U}(\varphi)$ given by $U=\mathcal{W}(\varphi)$, or

$$
\mathbb{S}_{(0)}=-\frac{1}{\kappa^{2}} \int d^{d} x \sqrt{-\gamma} \mathcal{W}
$$

As promised, we obtain (4.7) regardless of the sign of (3.3b). It follows that the leading asymptotics of the scalar field $\varphi^{I}$ is also determined, independently of the sign chosen in (3.3b), as we see in (4.8c). From (4.7) we can now determine the leading asymptotics of the fields by using the above flow equations, namely

$$
\begin{aligned}
e_{i}^{a}(r, x) & \sim e^{r} e_{(0) i}^{a}(x), & & \\
\Psi_{+i}(r, x) & \sim e^{r / 2} \Psi_{(0)+i}(x), & & \\
\dot{\varphi}^{I} & \sim \mathcal{G}^{I J} \partial_{J} \mathcal{W}, & & \text { or } \varphi^{I} \sim e^{-\mu^{I} r} \varphi_{(0)}^{I}, \\
\dot{\zeta}_{-}^{I} & \sim-\frac{1}{2} \zeta_{-}^{I}+\left(\mathcal{G}^{I K} \partial_{J} \partial_{K} \mathcal{W}\right) \zeta_{-}^{J}, & & \text { or } \zeta_{-}^{I} \sim e^{-\left(\mu^{I}+\frac{1}{2}\right) r} \zeta_{-(0)}^{I},
\end{aligned}
$$

where $\mu^{I}$ stands for the radial weight of $\varphi^{I}$ when the scalars are properly diagonalized.

\footnotetext{
${ }^{8}$ One might try to solve the HJ equation for the general scalar-gravity model by using the argument in [39].
} 
Now let us go to the next order of the bosonic sector. The HJ equation for $\mathbb{S}_{(2)}^{B}$ is then

$$
-\frac{2}{d-1} \mathcal{W} \gamma_{i j} \frac{\delta}{\delta \gamma_{i j}} \mathbb{S}_{(2)}^{B}+\mathcal{G}^{I J} \partial_{I} \mathcal{W} \frac{\delta}{\delta \varphi^{J}} \mathbb{S}_{(2)}^{B}+\frac{\sqrt{-\gamma}}{2 \kappa^{2}}\left(-R[\gamma]+\mathcal{G}_{I J} \partial_{i} \varphi^{I} \partial^{i} \varphi^{J}\right)=0 .
$$

The most general ansatz for $\mathbb{S}_{(2)}^{B}$ is as follows:

$$
\mathbb{S}_{(2)}^{B}=\frac{1}{\kappa^{2}} \int d^{d} x \sqrt{-\gamma}\left(\Xi(\varphi) R+A_{I J}(\varphi) \partial_{i} \varphi^{I} \partial^{i} \varphi^{J}\right)
$$

Then,

$$
\begin{aligned}
\gamma_{i j} \frac{\delta}{\delta \gamma_{i j}} \mathbb{S}_{(2)}^{B} & =\frac{\sqrt{-\gamma}}{\kappa^{2}} \frac{d-2}{2}\left(\Xi R+A_{I J} \partial_{i} \varphi^{I} \partial^{i} \varphi^{J}\right)-\frac{\sqrt{-\gamma}}{\kappa^{2}}(d-1) \square \Xi, \\
\frac{\delta}{\delta \varphi^{J}} \mathbb{S}_{(2)}^{B} & =\frac{\sqrt{-\gamma}}{\kappa^{2}}\left(R \partial_{J} \Xi+\partial_{J} A_{I K} \partial_{i} \varphi^{I} \partial^{i} \varphi^{K}-2 D_{i}\left(A_{J K} \partial^{i} \varphi^{K}\right)\right),
\end{aligned}
$$

where we used the relation

$$
\gamma^{i j} \delta R_{i j}=D^{i} D^{j} \delta \gamma_{i j}-\gamma^{i j} \square\left(\delta \gamma_{i j}\right)
$$

One can notice from (4.11) that

$$
\mathbb{L}_{(2)}^{B}=\frac{\sqrt{-\gamma}}{\kappa^{2}}\left(\Xi R+A_{I J} \partial_{i} \varphi^{I} \partial^{i} \varphi^{J}-\frac{2(d-1)}{d-2} \square \Xi\right) .
$$

Therefore, (4.9) becomes

$$
\begin{aligned}
0= & R\left(-\frac{d-2}{d-1} \mathcal{W} \Xi^{[1]}+\mathcal{G}^{I J} \partial_{I} \mathcal{W} \partial_{J} \Xi-\frac{1}{2}\right)+\partial_{i} \varphi^{I} \partial^{i} \varphi^{J}\left(-\frac{d-2}{d-1} \mathcal{W} A_{I J}+2 \mathcal{W} \partial_{I} \partial_{J} \Xi\right. \\
& \left.+\mathcal{G}^{K L} \partial_{L} \mathcal{W} \partial_{K} A_{I J}-2 \mathcal{G}^{K L} \partial_{K} \mathcal{W} \partial_{I} A_{L J}+\frac{1}{2} \mathcal{G}_{I J}\right)+2 \square \varphi^{I}\left(\mathcal{W} \partial_{I} \Xi-\mathcal{G}^{J K} \partial_{J} \mathcal{W} A_{I K}\right)
\end{aligned}
$$

and we obtain the equations for $\Xi$ and $A_{I J}$

$$
\begin{aligned}
& 0=-\frac{d-2}{d-1} \Xi+V^{I} \partial_{I} \Xi-\frac{1}{2 \mathcal{W}} \\
& 0=-\frac{d-2}{d-1} A_{I J}+V^{K} \partial_{K} A_{I J}+\partial_{I} V^{K} A_{J K}+\partial_{J} V^{K} A_{I K}+\frac{1}{2 \mathcal{W}} \mathcal{G}_{I J} \\
& 0=\partial_{I} \Xi-V^{J} A_{I J}
\end{aligned}
$$

where

$$
V^{I} \equiv \frac{1}{\mathcal{W}} \mathcal{G}^{I J} \partial_{J} \mathcal{W}
$$

Note that $A_{I J}$ should satisfy the condition

$$
\partial_{I}\left(V^{K} A_{J K}\right)=\partial_{J}\left(V^{K} A_{I K}\right) .
$$

We emphasize that we do not discuss the existence of a solution for $A_{I J}$ and $\Xi$ here. Nevertheless, equations $(4.16)$ are useful for determining $\mathbb{S}_{(1)}^{\zeta \zeta}, \mathbb{S}_{(2)}^{\Psi \Psi}$ and $\mathbb{S}_{(3 / 2)}^{\zeta \Psi}$. 
$\mathbb{S}_{(2 n)}^{B}(n \geq 2)$ is obtained by the following recursive equation

$$
\begin{aligned}
0= & -\frac{2}{d-1} \mathcal{W} \gamma^{i j} \pi_{(2 n) i j}^{B}+\mathcal{W} V^{I} \pi_{(2 n) I}^{B} \\
& +\frac{\kappa^{2}}{2 \sqrt{-\gamma}} \sum_{m=1}^{n-1}\left[4\left(\frac{1}{d-1} \gamma_{i j} \gamma_{k l}-\gamma_{i k} \gamma_{j l}\right) \pi_{B(2 m)}^{i j} \pi_{B(2 n-2 m)}^{k l}-\mathcal{G}^{I J} \pi_{I}^{B(2 m)} \pi_{J}^{B(2 n-2 m)}\right]
\end{aligned}
$$

In particular, when $d=4$ the inhomogeneous terms on the r.h.s. become

$$
2 \frac{\kappa^{2}}{\sqrt{-\gamma}}\left(\frac{1}{d-1} \gamma_{i j} \gamma_{k l}-\gamma_{i k} \gamma_{j l}\right) \pi_{(2)}^{i j} \pi_{(2)}^{k l}=\frac{\sqrt{-\gamma}}{\kappa^{2}} \Xi^{2}\left(\frac{d}{2(d-1)} R^{2}-2 R_{k l} R^{k l}\right)
$$

where

$$
\Xi=\frac{1}{2(d-2)}+\mathcal{O}\left(\varphi^{2}\right)
$$

is the solution of (4.16a), while other inhomogeneous terms are asymptotically suppressed.

\subsection{Fermionic sector}

After substituting the leading order solution (4.7) into the Hamiltonian constraint (3.9), we get the following first-order differential equation for $\breve{S} \equiv \mathbb{S}-\mathbb{S}_{(0)}$

$$
\begin{aligned}
& 0=\mathcal{W}\left(-\frac{1}{d-1} e_{i}^{a} \breve{\pi}_{a}^{i}+V^{I} \breve{\pi}_{I}^{\varphi}\right)-\frac{1}{2(d-1)} \mathcal{W}\left(\bar{\Psi}_{+i} \pi_{\Psi}^{i}+\pi_{\Psi}^{i} \Psi_{+i}\right)+\mathcal{W}\left(\frac{1}{2(d-1)} \delta_{I}^{J}+\partial_{I} V^{J}\right) \\
& \times\left(\bar{\zeta}_{-}^{I} \pi_{J}^{\zeta}+\pi_{J}^{\zeta} \zeta_{-}^{I}\right)+\frac{\kappa^{2}}{2 \sqrt{-\gamma}}\left\{\left(\frac{1}{d-1} e_{i}^{a} e_{j}^{b}-e_{j}^{a} e_{i}^{b}\right) \breve{\pi}_{a}^{i} \breve{\pi}_{b}^{j}-\mathcal{G}^{I J} \breve{\pi}_{I}^{\varphi} \breve{\pi}_{J}^{\varphi}+\mathcal{G}^{I J}\left(\pi_{I}^{\zeta} \not \triangleright D \pi_{J}^{\zeta}-\pi_{I}^{\zeta} \overleftarrow{\not D} \pi_{J}^{\zeta}\right)\right. \\
& -2 \breve{\pi}^{i j}\left[\left(\bar{\Psi}_{+i} \pi_{\Psi j}+\pi_{\Psi j} \Psi_{+i}\right)+\frac{1}{d-1} \pi_{\Psi}^{p}\left(\widehat{\Gamma}_{p i}-(d-2) \gamma_{p i}\right) \widehat{\Gamma}_{j}^{k} \Psi_{+k}\right. \\
& \left.+\frac{1}{d-1} \bar{\Psi}_{+k} \widehat{\Gamma}^{k}{ }_{j}\left(\widehat{\Gamma}_{i p}-(d-2) \gamma_{i p}\right) \pi_{\Psi}^{p}-i \frac{\partial_{I} \mathcal{W}}{\mathcal{W}} \gamma_{j k}\left(\bar{\zeta}_{-}^{I} \widehat{\Gamma}_{i} \pi_{\Psi}^{k}+\pi_{\Psi}^{k} \widehat{\Gamma}_{i} \zeta_{-}^{I}\right)\right] \\
& +\frac{2}{d-1} \gamma_{i j} \breve{\pi}^{i j}\left(-\bar{\zeta}_{-}^{I} \pi_{I}^{\zeta}-\pi_{I}^{\zeta} \zeta_{-}^{I}+\bar{\Psi}_{+k} \pi_{\Psi}^{k}+\pi_{\Psi}^{k} \Psi_{+k}-i \frac{\partial_{I} \mathcal{W}}{\mathcal{W}} \bar{\zeta}_{-}^{I} \widehat{\Gamma}_{k} \pi_{\Psi}^{k}-i \frac{\partial_{I} \mathcal{W}}{\mathcal{W}} \pi_{\Psi}^{k} \widehat{\Gamma}_{k} \zeta_{-}^{I}\right) \\
& +\left[\mathcal{G}^{I J} \mathcal{G}^{L M}\left(\partial_{J} \mathcal{G}_{M K}-\partial_{M} \mathcal{G}_{J K}\right)-\mathcal{W} \partial_{K}\left(\frac{\mathcal{G}^{I L}}{\mathcal{W}}\right)\right] \breve{\pi}_{I}^{\varphi}\left(\bar{\zeta}_{-}^{K} \pi_{L}^{\zeta}+\pi_{L}^{\zeta} \zeta_{-}^{K}\right) \\
& +i \breve{\pi}_{I}^{\varphi}\left[\frac{1}{d-1}\left(\bar{\zeta}_{-}^{I} \widehat{\Gamma}_{i} \pi_{\Psi}^{i}+\pi_{\Psi}^{i} \widehat{\Gamma}_{i} \zeta_{-}^{I}\right)\right. \\
& \left.-\mathcal{G}^{I J}\left(\pi_{J}^{\zeta} \widehat{\Gamma}^{i} \Psi_{+i}+\bar{\Psi}_{+i} \widehat{\Gamma}^{i} \pi_{J}^{\zeta}\right)\right]-\pi_{\Psi}^{k}\left[\left(\frac{1}{d-1} \widehat{\Gamma}_{k} \widehat{\Gamma}_{j}-\gamma_{k j}\right) \not \triangleright D-\overleftarrow{\not D}\left(\frac{1}{d-1} \widehat{\Gamma}_{k} \widehat{\Gamma}_{j}-\gamma_{k j}\right)\right] \pi_{\Psi}^{j} \\
& +\frac{i}{d-1}\left(\pi_{I}^{\zeta} \not \partial \varphi^{I} \widehat{\Gamma}_{i} \pi_{\Psi}^{i}-\pi_{\Psi}^{i} \widehat{\Gamma}_{i} \not \partial \varphi^{I} \pi_{I}^{\zeta}\right)-2 i \partial_{i} \varphi^{I}\left(\pi_{I}^{\zeta} \pi_{\Psi}^{i}-\pi_{\Psi}^{i} \pi_{I}^{\zeta}\right) \\
& \left.+\mathcal{G}^{I M} \mathcal{G}^{K N} \partial_{i} \varphi^{J}\left(\partial_{K} \mathcal{G}_{I J}-\partial_{I} \mathcal{G}_{K J}\right) \pi_{M}^{\zeta} \widehat{\Gamma}^{i} \pi_{N}^{\zeta}\right\} \\
& +\frac{\sqrt{-\gamma}}{2 \kappa^{2}}\left[-R[\gamma]+\mathcal{G}_{I J} \partial_{i} \varphi^{I} \partial^{i} \varphi^{J}+\mathcal{G}_{I J} \bar{\zeta}_{-}^{I}(\not D D-\overleftarrow{\mathbb{D}}) \zeta_{-}^{J}+\bar{\Psi}_{+i} \widehat{\Gamma}^{i j k}\left(\mathbb{D}_{j}-\overleftarrow{\mathbb{D}}_{j}\right) \Psi_{+k}\right. \\
& +D_{k}\left(\bar{\Psi}_{+i}\left(\gamma^{j k} \widehat{\Gamma}^{i}-\gamma^{i k} \widehat{\Gamma}^{j}\right) \Psi_{+j}\right)+i \mathcal{G}_{I J} \partial_{i} \varphi^{J}\left(\bar{\zeta}_{-}^{I} \widehat{\Gamma}^{j} \widehat{\Gamma}^{i} \Psi_{+j}-\bar{\Psi}_{+j} \widehat{\Gamma}^{i} \widehat{\Gamma}^{j} \zeta_{-}^{I}\right)
\end{aligned}
$$




$$
\begin{aligned}
& +\partial_{K} \mathcal{G}_{I J} \partial_{i} \varphi^{J}\left(\bar{\zeta}_{-}^{I} \widehat{\Gamma}^{i} \zeta_{-}^{K}-\bar{\zeta}_{-}^{K} \widehat{\Gamma}^{i} \zeta_{-}^{I}\right)-2 i V_{I}\left(\bar{\zeta}_{-}^{I} \widehat{\Gamma}^{i j} \mathbb{D}_{i} \Psi_{+j}+\bar{\Psi}_{+j} \overleftarrow{\mathbb{D}}_{i} \widehat{\Gamma}^{i j} \zeta_{-}^{I}\right) \\
& \left.+V_{I} \mathcal{G}_{J K} \partial_{i} \varphi^{J}\left(\bar{\zeta}_{-}^{I} \widehat{\Gamma}^{i} \zeta_{-}^{K}-\bar{\zeta}_{-}^{K} \hat{\Gamma}^{i} \zeta_{-}^{I}\right)\right]
\end{aligned}
$$

where

$$
\breve{\pi}_{a}^{i} \equiv \frac{\delta \breve{\mathbb{S}}}{\delta e_{i}^{a}}, \quad \breve{\pi}_{I}^{\varphi} \equiv \frac{\breve{\mathbb{S}}}{\delta \varphi^{I}}
$$

From this one could write a recursive equation for every $\mathbb{S}_{(k)}$. However, it looks too complicated, and thus we first write down the equations for $\mathbb{S}_{(1)}^{\zeta \zeta}, \mathbb{S}_{(3 / 2)}^{\zeta \Psi}$ and $\mathbb{S}_{(2)}^{\Psi \Psi}$, namely

$$
\begin{aligned}
0= & -\mathcal{W L} \mathbb{L}_{(1)}^{\zeta \zeta}+\mathcal{W}\left\{V^{I} \partial_{I}+\frac{1}{2(d-1)}\left(\bar{\zeta}_{-}^{I} \frac{\delta}{\delta \bar{\zeta}_{-}^{I}}+\frac{\overleftarrow{\delta}}{\delta \zeta_{-}^{I}} \zeta_{-}^{I}\right)+\partial_{I} V^{J}\left(\bar{\zeta}_{-}^{I} \frac{\delta}{\delta \bar{\zeta}_{-}^{J}}+\frac{\overleftarrow{\delta}}{\delta \zeta_{-}^{J}} \zeta_{-}^{I}\right)\right\} \mathbb{S}_{(1)}^{\zeta \zeta} \\
& +\frac{\sqrt{-\gamma}}{2 \kappa^{2}}\left[\mathcal{G}_{I J}\left(\bar{\zeta}_{-}^{I} \not D \zeta_{-}^{J}-\bar{\zeta}_{-}^{I} \overleftarrow{\not D} \zeta_{-}^{J}\right)+\left(V_{I} \mathcal{G}_{J K}+\partial_{K} \mathcal{G}_{I J}\right) \partial_{i} \varphi^{J}\left(\bar{\zeta}_{-}^{I} \widehat{\Gamma}^{i} \zeta_{-}^{K}-\bar{\zeta}_{-}^{K} \hat{\Gamma}^{i} \zeta_{-}^{I}\right)\right] . \quad(4.24 \mathrm{a}) \\
0= & -\frac{d-\frac{3}{2}}{d-1} \mathcal{W} \mathbb{L}_{(3 / 2)}^{\zeta \Psi}+\mathcal{W}\left[V^{I} \partial_{I}+\frac{1}{2(d-1)}\left(\bar{\zeta}_{-}^{I} \frac{\delta}{\delta \bar{\zeta}_{-}^{I}}+\frac{\overleftarrow{\delta}}{\delta \zeta_{-}^{I}} \zeta_{-}^{I}\right)+\partial_{L} V^{K}\left(\bar{\zeta}_{-}^{L} \frac{\delta}{\delta \bar{\zeta}_{-}^{K}}+\frac{\overleftarrow{\delta}}{\delta \zeta_{-}^{K}} \zeta_{-}^{L}\right)\right] \mathbb{S}_{(3 / 2)}^{\zeta \Psi} \\
& +\frac{\sqrt{-\gamma}}{2 \kappa^{2}} i\left[-2 V_{I}\left(\bar{\zeta}_{-}^{I} \widehat{\Gamma}^{i j} \mathbb{D}_{i} \Psi_{+j}+\bar{\Psi}_{+j} \overleftarrow{\mathbb{D}}_{i} \widehat{\Gamma}^{i j} \zeta_{-}^{I}\right)+\mathcal{G}_{I J}\left(\bar{\zeta}_{-}^{I} \widehat{\Gamma}^{j} \not \partial \varphi^{J} \Psi_{+j}-\bar{\Psi}_{+j} \not \partial \varphi^{J} \widehat{\Gamma}^{j} \zeta_{-}^{I}\right)\right],
\end{aligned}
$$

$$
\begin{aligned}
0= & -\frac{d-2}{d-1} \mathcal{W} \mathbb{L}_{(2)}^{\Psi \Psi}+\mathcal{G}^{I J} \partial_{I} \mathcal{W} \frac{\delta}{\delta \varphi^{J}} \mathbb{S}_{(2)}^{\Psi \Psi} \\
& +\frac{\sqrt{-\gamma}}{2 \kappa^{2}}\left[\bar{\Psi}_{+i} \widehat{\Gamma}^{i j k}\left(\mathbb{D}_{j}-\overleftarrow{\mathbb{D}}_{j}\right) \Psi_{+k}+D_{k}\left(\bar{\Psi}_{+i}\left(\gamma^{j k} \widehat{\Gamma}^{i}-\gamma^{i k} \widehat{\Gamma}^{j}\right) \Psi_{+j}\right)\right]
\end{aligned}
$$

where we used (3.27).

While (4.24a) and (4.24b) are not so easy to treat at first sight, the solution of (4.24c) is obvious once we take into account (4.16a), namely

$$
\mathbb{L}_{(2)}^{\Psi \Psi}=-\frac{\sqrt{-\gamma}}{\kappa^{2}} \Xi\left[\bar{\Psi}_{+i} \widehat{\Gamma}^{i j k}\left(\mathbb{D}_{j}-\overleftarrow{\mathbb{D}}_{j}\right) \Psi_{+k}+D_{k}\left(\bar{\Psi}_{+i}\left(\gamma^{j k} \widehat{\Gamma}^{i}-\gamma^{i k} \widehat{\Gamma}^{j}\right) \Psi_{+j}\right)\right] .
$$

Instead of solving (4.24a) and (4.24b) directly, we now try to solve the $\mathcal{F}_{+}$constraint (3.13), which requires much less effort. They are respectively at the order 1 and $3 / 2$

$$
\begin{gathered}
i \mathcal{G}^{I J} \partial_{I} \mathcal{W} \frac{\delta}{\delta \bar{\zeta}_{-}^{J}} \mathbb{S}_{(1)}^{\zeta \zeta}+\frac{1}{d-1} \mathcal{W} \widehat{\Gamma}_{i} \frac{\delta}{\delta \bar{\Psi}_{+i}} \mathbb{S}_{(3 / 2)}^{\zeta \Psi}=\frac{\sqrt{-\gamma}}{2 \kappa^{2}} i \mathcal{G}_{I J} \partial_{i} \varphi^{J} \hat{\Gamma}^{i} \zeta_{-}^{I}, \\
\frac{1}{d-1} \mathcal{W} \widehat{\Gamma}_{i} \frac{\delta}{\delta \bar{\Psi}_{+i}} \mathbb{S}_{(2)}^{\Psi \Psi}+i \mathcal{G}^{I J} \partial_{I} \mathcal{W} \frac{\delta}{\delta \bar{\zeta}_{-}^{J}} \mathbb{S}_{(3 / 2)}^{\zeta \Psi}=\frac{\sqrt{-\gamma}}{\kappa^{2}} \widehat{\Gamma}^{i j} \mathbb{D}_{i} \Psi_{+j} .
\end{gathered}
$$

The solution (4.25) allows us to solve (4.26b) immediately and we obtain

$$
\frac{\delta}{\delta \bar{\zeta}_{-}^{I}} \mathbb{S}_{(3 / 2)}^{\zeta \Psi}=i \frac{\sqrt{-\gamma}}{\kappa^{2}}\left(-2 \partial_{I} \Xi \bar{\zeta}_{-}^{I} \widehat{\Gamma}^{i j} \mathbb{D}_{i} \Psi_{+j}+A_{I J} \bar{\zeta}_{-}^{I} \widehat{\Gamma}^{i} \not \partial \varphi^{J} \Psi_{+i}\right)
$$

One can readily see that

$$
\begin{aligned}
\mathbb{S}_{(3 / 2)}^{\zeta \Psi}=\frac{i}{\kappa^{2}} \int d^{d} x \sqrt{-\gamma}[ & 2 \partial_{I} \Xi\left(\bar{\Psi}_{+i} \overleftarrow{\mathbb{D}}_{j} \widehat{\Gamma}^{i j} \zeta_{-}^{I}-\bar{\zeta}_{-}^{I} \widehat{\Gamma}^{i j} \mathbb{D}_{i} \Psi_{+j}\right) \\
& \left.+A_{I J}\left(\bar{\zeta}_{-}^{I} \widehat{\Gamma}^{i} \not \partial \varphi^{J} \Psi_{+i}-\bar{\Psi}_{+i} \not \partial \varphi^{I} \widehat{\Gamma}^{i} \zeta_{-}^{J}\right)\right] .
\end{aligned}
$$


In the same way, we find from (4.26a) that

$$
\mathbb{S}_{(1)}^{\zeta \zeta}=\frac{1}{\kappa^{2}} \int d^{d} x \sqrt{-\gamma}\left(A_{I J} \bar{\zeta}_{-}^{I}(\not D-\overleftarrow{\not D}) \zeta_{-}^{J}+\left(\partial_{J} A_{I k}-\partial_{I} A_{J K}\right) \bar{\zeta}_{-}^{I} \not \partial \varphi^{K} \zeta_{-}^{J}\right)
$$

Moreover, we can confirm that the solutions (4.29) and (4.28) satisfy the Hamiltonian constraints (4.24a) and (4.24b) respectively. That is not the whole story, however, and one has to convince themselves that the $\mathcal{F}_{-}=0$ constraint also holds for these solutions. From (3.13), we obtain

$$
\begin{aligned}
& 0=\mathbb{D}_{i} \frac{\delta}{\delta \bar{\Psi}_{+i}} \mathbb{S}_{(2 k)}^{\Psi \Psi}+\frac{i}{2} \partial_{i} \varphi^{I} \widehat{\Gamma}^{i} \frac{\delta}{\delta \bar{\zeta}_{-}^{I}} \mathbb{S}_{(2 k-1 / 2)}^{\zeta \Psi}-\widehat{\Gamma}_{i} \Psi+j \frac{\delta}{\delta \gamma_{i j}} \mathbb{S}_{(2 k)}^{B}, \\
& 0=\mathbb{D}_{i} \frac{\delta}{\delta \bar{\Psi}_{+i}} \mathbb{S}_{(2 k-1 / 2)}^{\zeta \Psi}+\frac{i}{2} \partial_{i} \varphi^{I} \widehat{\Gamma}^{i} \frac{\delta}{\delta \bar{\zeta}_{-}^{I}} \mathbb{S}_{(2 k-1)}^{\zeta \zeta}-\frac{i}{2} \zeta_{-}^{I} \frac{\delta}{\delta \varphi^{I}} \mathbb{S}_{(2 k)}^{B},
\end{aligned}
$$

where $k$ is an arbitrary positive integer. It is not so difficult to check that the solutions we obtained satisfy the constraints (4.30b) and (4.30a) for $k=1$, implying that the combination

is $\left(\epsilon_{+}\right)$supersymmetric.

$$
\mathbb{S}_{(2)}^{B}+\mathbb{S}_{(2)}^{\Psi \Psi}+\mathbb{S}_{(1)}^{\zeta \zeta}+\mathbb{S}_{(3 / 2)}^{\zeta \Psi}
$$

We have seen how to obtain Hamilton's principal function in the fermionic sector from its bosonic supersymmetric partner, but at the lowest order. It was relatively easy because we could give the most general ansatz for $\mathbb{S}_{(2)}^{B}$ which has a small number of terms. To go further we should first determine $\mathbb{S}_{(4)}^{B}, \mathbb{S}_{(6)}^{B}, \cdots$ and obtain their SUSY partners by using the above trick. The ansatz for $\mathbb{S}_{(2 n)}^{B}(n \geq 2)$, however, has lots of terms and is complicated, hence finding its SUSY partner is too tedious.

Although we stop finding the general solution of the HJ equations in the fermionic sector here, we remark that the solution we have found is almost sufficient for providing the divergent counterterms in the low dimensions, say, $d=4$. This is because in the generic case that there are no scalar fields dual to marginal operators, $\mathbb{S}_{(3)}^{\zeta \zeta}$ and $\mathbb{S}_{(7 / 2)}^{\zeta \Psi}$ are asymptotically suppressed in 4 dimensions. As a result, what remains in the case $d=4$ is only to determine $\mathbb{S}_{(4)}^{\Psi \Psi}$, corresponding to the logarithmically divergent terms, which are directly related to the holographic Weyl anomaly [13].

We should emphasize that from the general analysis here the divergent counterterms (except for $\mathbb{S}_{(0)}$ ) always satisfy the constraint $\mathcal{F}_{-}=0$ and so does the renormalized on-shell action $\widehat{S}_{\text {ren. }}$

We finish this subsection by presenting the recursive relation obtained from (3.12), namely

$$
\begin{aligned}
0= & -\frac{1}{d-1} \mathcal{W} \widehat{\Gamma}_{i} \pi_{\bar{\Psi}(n-1 / 2)}^{i}-i \mathcal{G}^{I J} \partial_{I} \mathcal{W} \pi_{J(n-1)}^{\bar{\zeta}}+\frac{\kappa^{2}}{\sqrt{-\gamma}} \sum_{m=1}^{\left\lfloor\frac{n}{2}\right\rfloor-1}\left[\frac{i}{2} \mathcal{G}^{I J} \pi_{I(2 m)}^{\varphi} \pi_{J(n-2 m-1)}^{\bar{\zeta}}\right. \\
& \left.+\pi_{(2 m)}^{j k}\left(\frac{1}{d-1} \gamma_{j k} \widehat{\Gamma}_{i}-\gamma_{i j} \widehat{\Gamma}_{k}\right) \pi_{\bar{\Psi}(n-2 m-1 / 2)}^{i}\right]
\end{aligned}
$$

where (integer or half-integer) $n \geq 4$. This will be useful for determining the super-Weyl anomaly in section 5.1.2. 


\subsection{Logarithmically divergent terms in $4 \mathrm{D}$}

As mentioned in subsection 3.2 , every $\mathbb{S}_{(k)}$ in the asymptotic expansion (3.25) with respect to the operator $\delta_{e}$ contains poles related to logarithmically divergent terms. Let us denote such terms by $\widetilde{\mathbb{S}}_{(k)}$. Whereas $\widetilde{\mathbb{S}}_{(4)}^{B}$ and $\widetilde{\mathbb{S}}_{(4)}^{\Psi \Psi}$ are purely gravitational (meaning that they are related only to the metric and the gravitino field) and universal, $\widetilde{\mathbb{S}}_{(1)}^{\zeta \zeta}, \widetilde{\mathbb{S}}_{(3 / 2)}^{\zeta \Psi}, \widetilde{\mathbb{S}}_{(2)}^{B}$ and $\widetilde{\mathbb{S}}_{(2)}^{\Psi \Psi}$ are model-dependent. We first discuss the former and then study the latter for a simple model.

$\widetilde{\mathbb{S}}_{(4)}^{B}$ is easily obtained from (4.19) and (4.20), namely

$$
\begin{aligned}
\widetilde{\mathbb{S}}_{(4)}^{B} & \equiv \int d^{d} x \sqrt{-\gamma} \widetilde{\mathcal{L}}_{(4)}^{B} \log e^{-2 r} \\
& =\frac{1}{4 \kappa^{2}(d-2)^{2}} \int d^{d} x \sqrt{-\gamma}\left(\frac{d}{4(d-1)} R^{2}-R_{i j} R^{i j}\right) \log e^{-2 r},
\end{aligned}
$$

which is already well-known. Meanwhile, $\widetilde{\mathbb{S}}_{(4)}^{\Psi \Psi}$ is determined by the inhomogeneous terms of the Hamiltonian constraint (4.22) at order 4, namely ${ }^{9}$

$$
\begin{aligned}
& \widetilde{\mathbb{S}}_{(4)}^{\Psi \Psi} \equiv \int d^{d} x \sqrt{-\gamma} \widetilde{\mathcal{L}}_{(4)}^{\Psi \Psi} \log e^{-2 r} \\
& =\int d^{d} x \frac{\kappa^{2}}{4 \sqrt{-\gamma}}\left\{2\left(\frac{1}{d-1} \gamma_{i j} \gamma_{k l}-\gamma_{i k} \gamma_{j l}\right) \widetilde{\pi}_{(2)}^{i j} e^{a k} \pi_{a(2)}^{l \Psi}-\widetilde{\pi}_{(2)}^{i j}\left(\bar{\Psi}_{+k} \widehat{\Gamma}_{j} \widehat{\Gamma}^{k} \pi_{\bar{\Psi} i}^{(2)}+\pi_{\Psi i}^{(2)} \widehat{\Gamma}^{k} \widehat{\Gamma}_{j} \Psi_{+k}\right)\right. \\
& -\frac{1}{d-1} \gamma_{i j} \widetilde{\pi}_{(2)}^{i j}\left(\bar{\Psi}_{+}^{k} \widehat{\Gamma}_{k l} \pi_{(2) \bar{\Psi}^{l}}^{l}+\pi_{\Psi}^{(2) l} \widehat{\Gamma}_{l k} \Psi_{+}^{k}\right)+\frac{1}{2(d-1)}\left(\pi_{\Psi}^{(2) k} \widehat{\Gamma}_{k} \not \widehat{\Gamma}_{j} \pi_{\bar{\Psi}}^{(2) j}-\pi_{\Psi}^{(2) k} \widehat{\Gamma}_{k} \overleftarrow{\not \supset} \widehat{\Gamma}_{j} \pi_{\bar{\Psi}}^{(2) j}\right) \\
& \left.+\frac{1}{2}\left(\pi_{\Psi}^{(2) i} \not \triangleright D \pi_{\bar{\Psi} i}^{(2)}-\pi_{\Psi}^{(2) i} \overleftarrow{\not \triangleright D} \pi_{\bar{\Psi} i}^{(2)}\right)\right\} \log e^{-2 r} \\
& =\frac{1}{8(d-2)^{2} \kappa^{2}} \int d^{d} x \sqrt{-\gamma}\left\{(d-3) R\left(\bar{\Psi}_{+i} \widehat{\Gamma}^{i j k} \mathbb{D}_{j} \Psi_{+k}-\bar{\Psi}_{+i} \overleftarrow{\mathbb{D}}_{j} \widehat{\Gamma}^{i j k} \Psi_{+k}\right)\right. \\
& +\frac{d}{d-1} R D_{j}\left[\bar{\Psi}_{+i}\left(\gamma^{i j} \widehat{\Gamma}^{k}-\gamma^{j k} \widehat{\Gamma}^{i}\right) \Psi_{+k}\right]-(d-4) R\left(\bar{\Psi}_{+i} \widehat{\Gamma}^{i} \widehat{\Gamma}^{j k} \mathbb{D}_{j} \Psi_{+k}-\bar{\Psi}_{+i} \overleftarrow{\mathbb{D}}_{j} \widehat{\Gamma}^{i j} \widehat{\Gamma}^{k} \Psi_{+k}\right) \\
& +\frac{(d-2)^{2}}{d-1} R\left[\bar{\Psi}_{+}^{k} \widehat{\Gamma}^{j} \mathbb{D}_{k} \Psi_{+j}-\bar{\Psi}_{+}^{i} \not \supset \Psi_{+i}-\bar{\Psi}_{+i} \overleftarrow{\mathbb{D}}^{k} \widehat{\Gamma}^{i} \Psi_{+k}+\bar{\Psi}_{+}^{k} \overleftarrow{\not D} \Psi_{+k}\right] \\
& +2 R_{k l}\left[\bar{\Psi}_{+i}\left[\left(\gamma^{i p} \widehat{\Gamma}^{k}-\gamma^{i k} \widehat{\Gamma}^{p}\right) \mathbb{D}^{l}-\overleftarrow{\mathbb{D}}^{l}\left(\gamma^{i p} \widehat{\Gamma}^{k}-\gamma^{p k} \widehat{\Gamma}^{i}\right)\right] \Psi_{+p}-\bar{\Psi}_{+i} \widehat{\Gamma}^{i} \widehat{\Gamma}^{j l} \mathbb{D}_{j} \Psi_{+}^{k}+\bar{\Psi}_{+}^{k} \overleftarrow{\mathbb{D}}_{j} \widehat{\Gamma}^{l j} \widehat{\Gamma}^{i} \Psi_{+i}\right. \\
& \left.-D_{j}\left[\bar{\Psi}_{+}^{l} \widehat{\Gamma}^{k j i} \Psi_{+i}-\bar{\Psi}_{+i} \widehat{\Gamma}^{i j k} \Psi_{+}^{l}-\bar{\Psi}_{+i}\left(\gamma^{j k} \gamma^{p l} \widehat{\Gamma}^{i}-\gamma^{j k} \gamma^{i l} \widehat{\Gamma}^{p}+\gamma^{j p} \gamma^{i l} \widehat{\Gamma}^{k}-\gamma^{p l} \gamma^{i j} \widehat{\Gamma}^{k}\right) \Psi_{+p}\right]\right] \\
& -\frac{2(d-2)^{2}}{d-1}\left(\bar{\Psi}_{+i} \overleftarrow{\mathbb{D}}_{j} \widehat{\Gamma}^{i j} \not \supset \widehat{\Gamma}^{k l} \mathbb{D}_{k} \Psi_{+l}-\bar{\Psi}_{+i} \overleftarrow{\mathbb{D}}_{j} \widehat{\Gamma}^{i j} \overleftarrow{\mathbb{D}} \widehat{\Gamma}^{k l} \mathbb{D}_{k} \Psi_{+l}\right) \\
& \left.-2\left(\bar{\Psi}_{+p} \overleftarrow{\mathbb{D}}_{q} \widehat{\Gamma}^{p q i} \not \supset \widehat{\Gamma}_{i}^{j k} \mathbb{D}_{j} \Psi_{+k}-\bar{\Psi}_{+p} \overleftarrow{\mathbb{D}}_{q} \widehat{\Gamma}^{p q i} \overleftarrow{D D}^{\Gamma_{i}}{ }^{j k} \mathbb{D}_{j} \Psi_{+k}\right)\right\} \log e^{-2 r}
\end{aligned}
$$

Although nontrivial, one can show that $\widetilde{\mathbb{S}}_{(4)}^{B}+\widetilde{\mathbb{S}}_{(4)}^{\Psi \Psi}$ satisfies the constraints $\mathcal{H}=\mathcal{F}_{-}=$

\footnotetext{
${ }^{9}$ When the boundary metric is flat, (4.34) matches with the result in [18].
} 
$\mathcal{F}_{+}=0$ (i.e. conformal, supersymmetry and super-Weyl invariance), namely

$$
\begin{aligned}
& 0=\left(e_{i}^{a} \frac{\delta}{\delta e_{i}^{a}}+\frac{1}{2} \bar{\Psi}_{+i} \frac{\delta}{\delta \bar{\Psi}_{+i}}+\frac{\overleftarrow{\delta}}{\delta \Psi_{+i}} \Psi_{+i}\right) \widetilde{\mathbb{S}}_{(4)}^{\Psi \Psi} \\
& 0=\widehat{\Gamma}_{i} \Psi_{+j} \frac{\delta}{\delta \gamma_{i j}} \widetilde{\mathbb{S}}_{(4)}^{B}+\mathbb{D}_{i} \frac{\delta}{\delta \bar{\Psi}_{+i}} \widetilde{\mathbb{S}}_{(4)}^{\Psi \Psi} \\
& 0=\widehat{\Gamma}_{i} \frac{\delta}{\delta \bar{\Psi}_{+i}} \mathbb{S}_{(4)}^{\Psi \Psi}
\end{aligned}
$$

\subsection{Generic finite counterterms in $4 \mathrm{D}$ and summary}

Up to now we obtained the generic part of the divergent counterterms. $\mathcal{S}_{\text {ct }}$ can involve additional finite terms which satisfy the first class constraints (3.14), though. ${ }^{10}$ The possible bosonic finite counterterms are the Euler density and the Weyl invariant in 4D, namely,

$$
E_{(4)}=\frac{1}{64}\left(R^{i j k l} R_{i j k l}-4 R^{i j} R_{i j}+R^{2}\right), \quad I_{(4)}=-\frac{1}{64}\left(R^{i j k l} R_{i j k l}-2 R^{i j} R_{i j}+\frac{1}{3} R^{2}\right) .
$$

The integral of the Euler density $E_{(4)}$ by itself satisfies all the first class constraints, since it is a topological quantity, any local variation of which vanishes. Therefore, we find that the possible supersymmetric finite counterterms are a linear combination of

$$
\begin{aligned}
X_{I} & =64 I_{(4)}+(d-3) R\left(\bar{\Psi}_{+i} \widehat{\Gamma}^{i j k} \mathbb{D}_{j} \Psi_{+k}-\bar{\Psi}_{+i} \overleftarrow{\mathbb{D}}_{j} \widehat{\Gamma}^{i j k} \Psi_{+k}\right) \\
& +\frac{d}{d-1} R D_{j}\left[\bar{\Psi}_{+i}\left(\gamma^{i j} \widehat{\Gamma}^{k}-\gamma^{j k} \widehat{\Gamma}^{i}\right) \Psi_{+k}\right] \\
& -(d-4) R\left(\bar{\Psi}_{+i} \widehat{\Gamma}^{i} \widehat{\Gamma}^{j k} \mathbb{D}_{j} \Psi_{+k}-\bar{\Psi}_{+i} \overleftarrow{\mathbb{D}}_{j} \widehat{\Gamma}^{i j} \widehat{\Gamma}^{k} \Psi_{+k}\right) \\
& +\frac{(d-2)^{2}}{d-1} R\left[\bar{\Psi}_{+}^{k} \widehat{\Gamma}^{j} \mathbb{D}_{k} \Psi_{+j}-\bar{\Psi}_{+}^{i} \not \mathbf{D} \Psi_{+i}-\bar{\Psi}_{+i} \overleftarrow{\mathbb{D}}^{k} \widehat{\Gamma}^{i} \Psi_{+k}+\bar{\Psi}_{+}^{k} \overleftarrow{\mathbb{D}} \Psi_{+k}\right] \\
& +2 R_{k l}\left[\bar{\Psi}_{+i}\left[\left(\gamma^{i p} \widehat{\Gamma}^{k}-\gamma^{i k} \widehat{\Gamma}^{p}\right) \mathbb{D}^{l}-\overleftarrow{\mathbb{D}}^{l}\left(\gamma^{i p} \widehat{\Gamma}^{k}-\gamma^{p k} \widehat{\Gamma}^{i}\right)\right] \Psi_{+p}-\bar{\Psi}_{+i} \widehat{\Gamma}^{i} \widehat{\Gamma}^{j l} \mathbb{D}_{j} \Psi_{+}^{k}+\bar{\Psi}_{+}^{k} \overleftarrow{\mathbb{D}}_{j} \widehat{\Gamma}^{l j} \widehat{\Gamma}^{i} \Psi_{+i}\right. \\
& \left.-D_{j}\left[\bar{\Psi}_{+}^{l} \widehat{\Gamma}^{k j i} \Psi_{+i}-\bar{\Psi}_{+i} \widehat{\Gamma}^{i j k} \Psi_{+}^{l}-\bar{\Psi}_{+i}\left(\gamma^{j k} \gamma^{p l} \widehat{\Gamma}^{i}-\gamma^{j k} \gamma^{i l} \widehat{\Gamma}^{p}+\gamma^{j p} \gamma^{i l} \widehat{\Gamma}^{k}-\gamma^{p l} \gamma^{i j} \widehat{\Gamma}^{k}\right) \Psi_{+p}\right]\right] \\
& -\frac{2(d-2)^{2}}{d-1}\left(\bar{\Psi}_{+i} \overleftarrow{\mathbb{D}}_{j} \widehat{\Gamma}^{i j} \not \supset \widehat{\Gamma}^{k l} \mathbb{D}_{k} \Psi_{+l}-\bar{\Psi}_{+i} \overleftarrow{\mathbb{D}}_{j} \widehat{\Gamma}^{i j} \overleftarrow{\mathbb{D}} \widehat{\Gamma}^{k l} \mathbb{D}_{k} \Psi_{+l}\right) \\
& -2\left(\bar{\Psi}_{+p} \overleftarrow{\mathbb{D}}_{q} \widehat{\Gamma}^{p q i} \not \supset \widehat{\Gamma}_{i}^{j k} \mathbb{D}_{j} \Psi_{+k}-\bar{\Psi}_{+p} \overleftarrow{\mathbb{D}}_{q} \widehat{\Gamma}^{p q i} \overleftarrow{\mathbb{D}} \widehat{\Gamma}_{i}^{j k} \mathbb{D}_{j} \Psi_{+k}\right),
\end{aligned}
$$

and

$$
X_{E}=E_{(4)}, \quad X_{P}=\mathcal{P}=\frac{1}{64} \epsilon^{i j k l} R_{i j p q} R_{k l}^{p q},
$$

where $\mathcal{P}$ is the Pontryagin density. Notice that the integral of $\mathcal{P}$ is a topological quantity and thus can be a finite counterterm as in the case of the Euler density, as long as there is no other symmetry which prevents its appearance.

\footnotetext{
${ }^{10}$ Otherwise, these finite terms would generate trivial cocycle terms, which do not have any physical implication.
} 
In summary, collecting all of these finite counterterms and the previous divergent ones we obtain

$$
\begin{aligned}
& S_{\mathrm{ct}}=-\left(\mathbb{S}_{(0)}+\mathbb{S}_{(1)}+\mathbb{S}_{(2)}\right)-\left(\widetilde{\mathbb{S}}_{(4)}^{B}+\widetilde{\mathbb{S}}_{(4)}^{\Psi \Psi}\right)+\cdots \\
&=\frac{1}{\kappa^{2}} \int d^{d} x \sqrt{-\gamma}\left\{\mathcal{W}-\Xi R-A_{I J} \partial_{i} \varphi^{I} \partial^{i} \varphi^{J}-A_{I J} \bar{\zeta}_{-}^{I}(\not \triangleright D-\overleftarrow{\mathbb{D}}) \zeta_{-}^{J}\right. \\
& \quad-\left(\partial_{J} A_{I K}-\partial_{I} A_{J K}\right) \bar{\zeta}_{-}^{I} \not \partial \varphi^{K} \zeta_{-}^{J}-2 i \partial_{I} \Xi\left(\bar{\Psi}_{+i} \overleftarrow{\mathbb{D}}_{j} \widehat{\Gamma}^{i j} \zeta_{-}^{I}-\bar{\zeta}_{-} \widehat{\Gamma}^{i j} \mathbb{D}_{i} \Psi_{+j}\right) \\
& \quad-i A_{I J}\left(\bar{\zeta}_{-}^{I} \widehat{\Gamma}^{i} \not \partial \varphi^{J} \Psi_{+i}-\bar{\Psi}_{+i} \not \partial \varphi^{I} \widehat{\Gamma}^{i} \zeta_{-}^{J}\right)+\Xi \bar{\Psi}_{+i} \widehat{\Gamma}^{i j k}\left(\mathbb{D}_{j}-\overleftarrow{\mathbb{D}}_{j}\right) \Psi_{+k} \\
&\left.\quad \quad \bar{\Psi}_{+i}\left(\partial^{i} \Xi \widehat{\Gamma}^{j}-\partial^{j} \Xi \widehat{\Gamma}^{i}\right) \Psi_{+j}\right\} \\
& \quad-\widetilde{\mathbb{S}}_{(4)}^{B}-\widetilde{\mathbb{S}}_{(4)}^{\Psi \Psi}+\frac{1}{\kappa^{2}} \int d^{d} x \sqrt{-\gamma}\left(\alpha_{I} X_{I}+\alpha_{E} X_{E}+\alpha_{P} X_{P}\right)+\cdots
\end{aligned}
$$

where $\widetilde{\mathbb{S}}_{(4)}^{B}$ and $\widetilde{\mathbb{S}}_{(4)}^{\Psi \Psi}$ are given in (4.33) and (4.34) and $\alpha_{I}, \alpha_{E}$ and $\alpha_{P}$ are arbitrary constants. Here the ellipses stand for model-dependent terms, which we discuss in section 4.5 for a simple toy model.

\subsection{Application to a toy model}

For completeness, we present an application of our general procedure to a simple toy model.

In the toy model there is only one scalar field, which corresponds to an operator with the scaling dimension $\Delta=d-1$ with $d=4$. In principle, there are two possibilities for the coefficient of $\varphi^{2}$-term of the bulk superpotential $\mathcal{W} ;-\frac{1}{2}$ corresponds to deformation, and $-\frac{d-1}{2}$ corresponds to RG-flow due to giving a VEV [49]. However, as we have seen, SUSY requires that the counterterm should be the same as the bulk superpotential $\mathcal{W}$, and thus we just need to consider the $-\frac{1}{2}$ case. It follows that

$$
\mathcal{W}=-(d-1)-\frac{1}{2} \varphi^{2}+k_{3} \varphi^{3}+k_{4} \varphi^{4}+\mathcal{O}\left(\varphi^{5}\right)
$$

where $k_{3}$ and $k_{4}$ are arbitrary constants, and therefore the solution of (4.16a), (4.16b) and (4.16c) becomes

$$
\Xi=\frac{1}{2(d-2)}-\frac{1}{d-4} \cdot \frac{1}{4(d-1)} \varphi^{2}+\cdots, \quad A_{I J}=-\frac{1}{d-4} \cdot \frac{1}{2}+\cdots .
$$

The divergent counterterms that we need, other than those in (4.39), are only the logarithmically divergent terms. Following the argument in section 3.2 again we can determine them from the poles (when $d=4$ ) in $\Xi$ and $A_{I J}$ and are responsible for additional logarithmically divergent terms.

We thus obtain

$$
\begin{aligned}
& \widetilde{\mathbb{S}}_{(2)}^{B}=\frac{1}{4 \kappa^{2}} \int d^{d} x \sqrt{-\gamma}\left(\frac{1}{2(d-1)} \varphi^{2} R+\partial_{i} \varphi \partial^{i} \varphi\right) \log e^{-2 r}, \\
& \widetilde{\mathbb{S}}_{(1)}^{\zeta \zeta}=\frac{1}{4 \kappa^{2}} \int d^{d} x \sqrt{-\gamma}\left(\bar{\zeta}_{-} \not \supset \zeta_{-}+\text {h.c. }\right) \log e^{-2 r}
\end{aligned}
$$




$$
\begin{aligned}
\widetilde{\mathbb{S}}_{(3 / 2)}^{\zeta \Psi} & =\frac{i}{4 \kappa^{2}} \int d^{d} x \sqrt{-\gamma}\left(\bar{\zeta}_{-} \widehat{\Gamma}^{i \not \partial \varphi} \varphi \Psi_{+i}-\frac{2}{(d-1)} \varphi \bar{\zeta}_{-} \widehat{\Gamma}^{i j} \mathbb{D}_{i} \Psi_{+j}+\text { h.c. }\right) \log e^{-2 r} \\
\widetilde{\mathbb{S}}_{(2)}^{\Psi \Psi} & =-\frac{1}{4 \kappa^{2}} \int d^{d} x \sqrt{-\gamma} \frac{1}{d-1}\left(\frac{1}{2} \varphi^{2} \bar{\Psi}_{+i} \widehat{\Gamma}^{i j k} \mathbb{D}_{j} \Psi_{+k}+\varphi \bar{\Psi}_{+i} \partial^{i} \varphi \widehat{\Gamma}^{j} \Psi_{+j}+\text { h.c. }\right) \log e^{-2 r}
\end{aligned}
$$

One can easily check that $\widetilde{\mathbb{S}}_{(2)}^{B}+\widetilde{\mathbb{S}}_{(1)}^{\zeta \zeta}+\widetilde{\mathbb{S}}_{(3 / 2)}^{\zeta \Psi}+\widetilde{\mathbb{S}}_{(2)}^{\Psi \Psi}$ again satisfies the constraints $\mathcal{H}=$ $\mathcal{F}_{-}=\mathcal{F}_{+}=0$.

Besides $X_{I}$ and $X_{E}$, the possible finite counterterms (conformal and $\epsilon_{+}$supersymmetric) are

$$
\begin{aligned}
X_{0}= & \frac{1}{2(d-1)} \varphi^{2} R+\partial_{i} \varphi \partial^{i} \varphi+\bar{\zeta}_{-} \not D \zeta_{-}+i \bar{\zeta}_{-} \widehat{\Gamma}^{i} \not \partial \varphi \Psi_{+i} \\
& -\frac{2 i}{d-1} \varphi \bar{\zeta}_{-} \widehat{\Gamma}^{i j} \mathbb{D}_{i} \Psi_{+j}-\frac{1}{2(d-1)} \varphi^{2} \bar{\Psi}_{+i} \widehat{\Gamma}^{i j k} \mathbb{D}_{j} \Psi_{+k}-\frac{1}{d-1} \varphi \bar{\Psi}_{+i} \partial^{i} \varphi \widehat{\Gamma}^{j} \Psi_{+j}+\text { h.c. }
\end{aligned}
$$

and the finite term $k_{4} \varphi^{4}$ in $\mathcal{W}$ should be in the counterterms without any ambiguity, due to the $\mathcal{F}_{-}$constraint.

In total, the divergent counterterms for the toy model are

$$
\mathbb{S}_{\mathrm{ct}}^{d i v}=-\left(\mathbb{S}_{(0)}+\mathbb{S}_{(1)}+\mathbb{S}_{(3 / 2)}+\mathbb{S}_{(2)}\right)-\int d^{d} x \sqrt{-\gamma} \widetilde{\mathcal{L}}_{[4]} \log e^{-2 r},
$$

where the logarithmically divergent counterterms are

$$
\int d^{d} x \sqrt{-\gamma} \widetilde{\mathcal{L}}_{[4]} \log e^{-2 r}=\widetilde{\mathbb{S}}_{(1)}^{\zeta \zeta}+\widetilde{\mathbb{S}}_{(3 / 2)}^{\zeta \Psi}+\widetilde{\mathbb{S}}_{(2)}^{B}+\widetilde{\mathbb{S}}_{(2)}^{\Psi \Psi}+\widetilde{\mathbb{S}}_{(4)}^{B}+\widetilde{\mathbb{S}}_{(4)}^{\Psi \Psi}
$$

Adding possible finite ones, the whole counterterms are

$$
\begin{aligned}
\mathbb{S}_{\mathrm{ct}}= & \frac{1}{\kappa^{2}} \int d^{d} x \sqrt{-\gamma}\left[-(d-1)-\frac{1}{2} \varphi^{2}+k_{3} \varphi^{3}+k_{4} \varphi^{4}-\frac{1}{2(d-2)} R\right. \\
& \left.+\frac{1}{2(d-2)} \bar{\Psi}_{+i} \widehat{\Gamma}^{i j k}\left(\mathbb{D}_{j}-\overleftarrow{\mathbb{D}}_{j}\right) \Psi_{+k}\right]-\int d^{d} x \sqrt{-\gamma} \widetilde{\mathcal{L}}_{[4]} \log e^{-2 r} \\
& +\frac{1}{\kappa^{2}} \int d^{d} x \sqrt{-\gamma}\left(\alpha_{I} X_{I}+\alpha_{E} X_{E}+\alpha_{P} X_{P}+\alpha_{0} X_{0}\right),
\end{aligned}
$$

where $\alpha_{E}, \alpha_{I}, \alpha_{P}$ and $\alpha_{0}$ are arbitrary constants and determine the renormalization scheme.

\section{$5 \quad$ Holographic dictionary and Ward identities}

Now that all the counterterms are determined, we can relate by the holographic dictionary [12] the renormalized canonical momenta to the renormalized local operators of the boundary field theory, namely

$$
\mathcal{T}_{a}^{i}=-\lim _{r \rightarrow \infty} e^{(d+1) r} \frac{1}{\sqrt{-\gamma}}\left(\pi_{a}^{i}+\frac{\delta S_{\mathrm{ct}}}{\delta e_{i}^{a}}\right):=-\frac{1}{\left|\boldsymbol{e}_{(0)}\right|} \Pi_{a}^{i}
$$




$$
\begin{aligned}
& \mathcal{O}_{I}^{\varphi}=\lim _{r \rightarrow \infty} e^{\left(d+\mu^{I}\right) r} \frac{1}{\sqrt{-\gamma}}\left(\pi_{I}^{\varphi}+\frac{\delta S_{\mathrm{ct}}}{\delta \varphi^{I}}\right):=\frac{1}{\left|\boldsymbol{e}_{(0)}\right|} \Pi_{I}^{\varphi}, \\
& \mathcal{O}_{I}^{\bar{\zeta}}=\lim _{r \rightarrow \infty} e^{\left(d+\mu^{I}+\frac{1}{2}\right) r} \frac{1}{\sqrt{-\gamma}}\left(\pi_{I}^{\bar{\zeta}}+\frac{\delta S_{\mathrm{ct}}}{\delta \bar{\zeta}^{I}}\right):=\frac{1}{\left|\boldsymbol{e}_{(0)}\right|} \Pi_{I}^{\bar{\zeta}}, \\
& \mathcal{S}^{i}=\lim _{r \rightarrow \infty} e^{\left(d+\frac{1}{2}\right) r} \frac{1}{\sqrt{-\gamma}}\left(\pi_{\bar{\Psi}}^{i}+\frac{\delta S_{\mathrm{ct}}}{\delta \bar{\Psi}_{+i}}\right):=\frac{1}{\left|\boldsymbol{e}_{(0)}\right|} \Pi_{\bar{\Psi}}^{i},
\end{aligned}
$$

where $\mathcal{T}_{a}^{i}$ is the energy-momentum tensor, ${ }^{11} \mathcal{S}^{i}$ is the supercurrent ${ }^{12}$ and $\boldsymbol{e}_{(0)}=\operatorname{det}\left(e_{(0) i}^{a}\right)$. We note that since these local renormalized operators are obtained in the presence of arbitrary sources we can obtain higher-point functions simply by taking functional derivatives of them with respect to the sources.

\subsection{Ward identities and anomalies}

One can find from the computation of section 4 and 4.5 that $\mathbb{S}_{\mathrm{ct}}$ satisfies the first class constraints $\mathcal{H}^{i}=\mathcal{F}_{-}=0$ and the local Lorentz constraint (3.7), and so does the renormalized on-shell action $\widehat{S}_{\text {ren }}$. This is also related to the fact that these constraints are linear functional derivative equations.

Since $\mathcal{H}$ and $\mathcal{F}_{+}$are not linear constraints, one should expect that the counterterms do not satisfy the constraints $\mathcal{H}=0$ and $\mathcal{F}_{+}=0$ in general and thus generate non-trivial cocycle terms, which appear in the constraints for the renormalized on-shell action. Also, the poles appearing in solving the constraints contribute to the corresponding anomaly. In total, after removing all divergent counterterms, the first class constraints (3.12), (3.13), (3.9), (3.10) and (3.15) are reduced into

$$
\begin{aligned}
0= & -\frac{1}{2} \Gamma^{a} \Psi_{(0)+i} \mathcal{T}_{a}^{i}+\frac{i}{2} \zeta_{(0)-}^{I} \mathcal{O}_{I}^{\varphi}-\frac{i}{2} \not \varphi_{(0)}^{I} \mathcal{O}_{I}^{\bar{\zeta}}-\mathbb{D}_{i} \mathcal{S}^{i}, \\
\mathcal{A}_{\mathrm{sW}}= & -i \mathcal{G}^{I J} \partial_{I} \mathcal{W} \mathcal{O}_{J}^{\bar{\zeta}}+\widehat{\Gamma}_{i} \mathcal{S}^{i}, \\
\mathcal{A}_{\mathrm{W}}= & e_{(0) i}^{a} \mathcal{T}_{a}^{i}-\mathcal{G}^{I J} \partial_{I} \mathcal{W} \mathcal{O}_{J}^{\varphi}-\frac{1}{2}\left(\bar{\Psi}_{(0)+i} S^{i}+\text { h.c. }\right) \\
& +\left(\frac{1}{2} \delta_{I}^{J}-\partial_{I} \partial^{J} \mathcal{W}\right)\left(\bar{\zeta}_{(0)-}^{I} \mathcal{O}_{J}^{\bar{\zeta}}+\text { h.c. }\right), \\
0= & e_{(0)}^{a i} D_{j} \mathcal{T}_{a}^{j}+\partial^{i} \varphi_{(0)}^{I} \mathcal{O}_{I}^{\varphi}+\left(\bar{\zeta}_{(0)-}^{I} \overleftarrow{\mathbb{D}}^{i} \mathcal{O}_{I}^{\bar{\zeta}}+\text { h.c. }\right)+\left(\bar{\Psi}_{(0)+j} \overleftarrow{\mathbb{D}}^{i} \mathcal{S}^{j}+\text { h.c. }\right) \\
& -D_{j}\left(\bar{\Psi}_{(0)+}^{i} \mathcal{S}^{j}+\text { h.c. }\right), \\
0= & e_{(0) a i} \mathcal{T}_{b}^{i}-e_{(0) b i} \mathcal{T}_{a}^{i}+\frac{1}{2}\left(\bar{\zeta}_{(0)-}^{I} \Gamma_{a b} \mathcal{O}_{I}^{\zeta}+\bar{\Psi}_{(0)+i} \Gamma_{a b} \mathcal{S}^{i}+\text { h.c. }\right),
\end{aligned}
$$

where $\mathcal{A}_{\mathrm{sW}}$ and $\mathcal{A}_{\mathrm{W}}$ are the super-Weyl and Weyl anomaly densities respectively. In (5.2) we keep only up to quadratic order and zero order in $\varphi^{I}$ in the Taylor expansion of $\mathcal{W}$ and $\mathcal{G}_{I J}$ respectively.

\footnotetext{
${ }^{11}$ The definition of the energy-momentum tensor is modified when the vielbein is used instead of the metric, see e.g. (2.198) in [50].

${ }^{12}$ The spinor index of the supercurrent $\mathcal{S}^{i}$ is implicit.
} 
We identify the constraints (5.2) with the Ward identities, which relate the local sources and their dual operators of the field theory. These Ward identities play a key role in the following discussion and reflect the remaining local symmetries of the bulk SUGRA after fixing the strong FG gauge (B.14), on which we did HR for the bulk theory in section 4. The remaining local symmetry transformations of SUGRA are called generalized PenroseBrown-Henneaux (gPBH) transformations, whose action on the sources is carefully treated in appendix B.4. The resulting expressions are (B.38). Before discussing the gPBH action on the renormalized canonical momenta, let us first determine the anomalies explicitly in the case of $d=4$.

\subsubsection{Weyl anomaly}

Although there are many ways to find the Weyl anomaly, a direct way is to read it from the HJ equation. One can see that in (4.22) at order 4 the first linear terms are indeed the r.h.s. of the trace Ward identity (5.2c) and the rest of the terms give part of the trace anomaly. The terms with the pole $1 /(d-4)$ which appeared in the HJ equations for $\mathbb{S}_{(1)}$, $\cdots, \mathbb{S}_{(4)}$ are also inherited into $(4.22)$ for $\mathbb{S}_{[4]}$. These non-homogeneous terms are already identified with the logarithmically divergent terms and thus we only need to multiply them by 2 to obtain the trace anomaly [8]. For the metric and gravitino parts the trace anomaly density is ${ }^{13}$

$$
\begin{aligned}
& \mathcal{A}_{\mathrm{W}}^{(G)}\left[e, \Psi_{+}\right]=\frac{1}{4(d-2)^{2} \kappa^{2}}\left\{\frac{d}{2(d-1)} R^{2}-2 R_{i j} R^{i j}\right. \\
& +(d-3) R\left(\bar{\Psi}_{+i} \widehat{\Gamma}^{i j k} \mathbb{D}_{j} \Psi_{+k}-\bar{\Psi}_{+i} \overleftarrow{\mathbb{D}}_{j} \widehat{\Gamma}^{i j k} \Psi_{+k}\right) \\
& +\frac{d}{d-1} R D_{j}\left[\bar{\Psi}_{+i}\left(\gamma^{i j} \widehat{\Gamma}^{k}-\gamma^{j k} \widehat{\Gamma}^{i}\right) \Psi_{+k}\right] \\
& -(d-4) R\left(\bar{\Psi}_{+i} \widehat{\Gamma}^{i} \widehat{\Gamma}^{j k} \mathbb{D}_{j} \Psi_{+k}-\bar{\Psi}_{+i} \overleftarrow{\mathbb{D}}_{j} \widehat{\Gamma}^{i j} \widehat{\Gamma}^{k} \Psi_{+k}\right) \\
& +\frac{(d-2)^{2}}{d-1} R\left[\bar{\Psi}_{+}^{k} \widehat{\Gamma}^{j} \mathbb{D}_{k} \Psi_{+j}-\bar{\Psi}_{+}^{i} \not D D \Psi_{+i}-\bar{\Psi}_{+i} \overleftarrow{\mathbb{D}}^{k} \widehat{\Gamma}^{i} \Psi_{+k}+\bar{\Psi}_{+}^{k} \overleftarrow{\not D} \Psi_{+k}\right] \\
& +2 R_{k l}\left[\bar{\Psi}_{+i}\left[\left(\gamma^{i p} \widehat{\Gamma}^{k}-\gamma^{i k} \widehat{\Gamma}^{p}\right) \mathbb{D}^{l}-\overleftarrow{\mathbb{D}}^{l}\left(\gamma^{i p} \widehat{\Gamma}^{k}-\gamma^{p k} \widehat{\Gamma}^{i}\right)\right] \Psi_{+p}\right. \\
& -\bar{\Psi}_{+i} \widehat{\Gamma}^{i} \widehat{\Gamma}^{j l} \mathbb{D}_{j} \Psi_{+}^{k}+\bar{\Psi}_{+}^{k} \overleftarrow{\mathbb{D}}_{j} \widehat{\Gamma}^{l j} \widehat{\Gamma}^{i} \Psi_{+i}-D_{j}\left[\bar{\Psi}_{+}^{l} \widehat{\Gamma}^{k j i} \Psi_{+i}-\bar{\Psi}_{+i} \widehat{\Gamma}^{i j k} \Psi_{+}^{l}\right. \\
& \left.\left.-\bar{\Psi}_{+i}\left(\gamma^{j k} \gamma^{p l} \widehat{\Gamma}^{i}-\gamma^{j k} \gamma^{i l} \widehat{\Gamma}^{p}+\gamma^{j p} \gamma^{i l} \widehat{\Gamma}^{k}-\gamma^{p l} \gamma^{i j} \widehat{\Gamma}^{k}\right) \Psi_{+p}\right]\right] \\
& -\frac{2(d-2)^{2}}{d-1}\left(\bar{\Psi}_{+i} \overleftarrow{\mathbb{D}}_{j} \widehat{\Gamma}^{i j} \not \supset \widehat{\Gamma}^{k l} \mathbb{D}_{k} \Psi_{+l}-\bar{\Psi}_{+i} \overleftarrow{\mathbb{D}}_{j} \widehat{\Gamma}^{i j} \overleftarrow{\mathbb{D}} \widehat{\Gamma}^{k l} \mathbb{D}_{k} \Psi_{+l}\right) \\
& \left.-2\left(\bar{\Psi}_{+p} \overleftarrow{\mathbb{D}}_{q} \widehat{\Gamma}^{p q i} \not \supset \widehat{\Gamma}_{i}^{j k} \mathbb{D}_{j} \Psi_{+k}-\bar{\Psi}_{+p} \overleftarrow{\mathbb{D}}_{q} \widehat{\Gamma}^{p q i} \overleftarrow{\not D} \widehat{\Gamma}_{i}^{j k} \mathbb{D}_{j} \Psi_{+k}\right)\right\}
\end{aligned}
$$

The holographic computation of the supersymmetric Weyl anomaly in $4 \mathrm{D}$ is quite remarkable; even though its bosonic part has already been known for a long time, it seems

\footnotetext{
${ }^{13}$ The SUSY completion of the Weyl anomaly in a 4 dimensional supersymmetric theory was obtained in $[51,52]$ by using the superspace formalism. To get the fermionic sector explicitly, however, one has yet to expand it further around the bosonic coordinates.
} 
really tough to obtain its SUSY partner terms by means of giving an ansatz and finding out the coefficients, whereas holography enables us to compute them directly.

We comment that although the bosonic sector of $\mathcal{A}_{\mathrm{W}}^{G}$ is the sum of the a anomaly density $E_{(4)}$ and $c$ anomaly $I_{(4)}$, the fermionic sector is in fact the SUSY partner of the $c$ anomaly density up to a total derivative. This is because the integral of $E_{(4)}$ is supersymmetric by itself, as mentioned before.

For the toy model of section 4.5, we have an additional contribution to the Weyl anomaly density, which is

$$
\begin{aligned}
\mathcal{A}_{W}^{(\text {model })}[\Phi]=\frac{1}{2 \kappa^{2}}( & \frac{1}{2(d-1)} \varphi^{2} R+\partial_{i} \varphi \partial^{i} \varphi+\bar{\zeta}_{-} \not \supset \zeta_{-}+i \bar{\zeta}_{-} \widehat{\Gamma}^{i} \not \partial \varphi \Psi_{+i}-\frac{2 i}{d-1} \varphi \bar{\zeta}_{-} \widehat{\Gamma}^{i j} \mathbb{D}_{i} \Psi_{+j} \\
& \left.-\frac{1}{2(d-1)} \varphi^{2} \bar{\Psi}_{+i} \widehat{\Gamma}^{i j k} \mathbb{D}_{j} \Psi_{+k}-\frac{1}{d-1} \varphi \bar{\Psi}_{+i} \partial^{i} \varphi \widehat{\Gamma}^{j} \Psi_{+j}+\text { h.c. }\right)
\end{aligned}
$$

The total Weyl anomaly density is thus given by ${ }^{14}$

$$
\begin{aligned}
\mathcal{A}_{W}[\Phi]= & \mathcal{A}_{W}^{(G)}[\Phi]+\mathcal{A}_{W}^{(\text {model })}[\Phi] \\
= & \mathcal{A}_{W}^{(G)}+\frac{1}{2 \kappa^{2}}\left(\frac{1}{2(d-1)} \varphi^{2} R+\partial_{i} \varphi \partial^{i} \varphi+\bar{\zeta}_{-} \not \boldsymbol{D}_{-}+i \bar{\zeta}_{-} \widehat{\Gamma}^{i} \not \varphi \varphi \Psi_{+i}\right. \\
& \left.-\frac{2 i}{d-1} \varphi \bar{\zeta}_{-} \widehat{\Gamma}^{i j} \mathbb{D}_{i} \Psi_{+j}-\frac{1}{2(d-1)} \varphi^{2} \bar{\Psi}_{+i} \widehat{\Gamma}^{i j k} \mathbb{D}_{j} \Psi_{+k}-\frac{1}{d-1} \varphi \bar{\Psi}_{+i} \partial^{i} \varphi \widehat{\Gamma}^{j} \Psi_{+j}+\text { h.c. }\right) .
\end{aligned}
$$

\subsubsection{Super-Weyl anomaly}

Here we compute the super-Weyl anomaly for the toy model. As pointed out in section $4.2,(4.26 \mathrm{~b})$ holds up to the finite order. For the toy model, it means that the r.h.s. of $(4.26 \mathrm{~b})$ is not canceled out and an additional finite term

$$
+\frac{\sqrt{-\gamma}}{\kappa^{2}} \frac{\varphi^{2}}{2(d-1)} \widehat{\Gamma}^{i j} \mathbb{D}_{i} \Psi_{+j}
$$

comes out from the 1.h.s. of (4.26b). As in the case of the Weyl anomaly, we thus get from (4.32)

$$
\begin{aligned}
& -i \mathcal{G}^{I J} \partial_{J} \mathcal{W} \pi_{(7 / 2) I}^{\bar{\zeta}}-\frac{1}{d-1} \mathcal{W} \widehat{\Gamma}_{i} \pi_{(4) \bar{\Psi}}^{i} \\
= & -\frac{\kappa^{2}}{\sqrt{-\gamma}} \pi_{(2)}^{j k}\left(\frac{1}{d-1} \gamma_{j k} \widehat{\Gamma}_{i}-\gamma_{i j} \widehat{\Gamma}_{k}\right) \pi_{(2) \bar{\Psi}}^{i}-\frac{\sqrt{-\gamma}}{2 \kappa^{2}} i \partial_{i} \varphi \widehat{\Gamma}^{i} \zeta_{-}+\frac{\sqrt{-\gamma}}{\kappa^{2}} \frac{\varphi^{2}}{2(d-1)} \widehat{\Gamma}^{i j} \mathbb{D}_{i} \Psi_{+j} \\
= & \frac{\sqrt{-\gamma}}{\kappa^{2}}\left[\frac{1}{4(d-2)^{2}}\left(\frac{d}{d-1} R \widehat{\Gamma}^{k l}-2 R_{i}{ }^{k} \widehat{\Gamma}^{i l}+2 R_{i} \widehat{\Gamma}^{i k}\right) \mathbb{D}_{k} \Psi_{+l}-\frac{i}{2} \partial_{i} \varphi \widehat{\Gamma}^{i} \zeta_{-}+\frac{1}{2(d-1)} \varphi^{2} \widehat{\Gamma}^{i j} \mathbb{D}_{i} \Psi_{+j}\right],
\end{aligned}
$$

\footnotetext{
${ }^{14}$ It seems that the bosonic sector of the conformal anomaly density $\mathcal{A}$ here is different from the one given in [8] (see (162) there), because of the $\varphi^{4}$ term in $\widetilde{\mathcal{L}}_{(4)}$. However, one can easily check that it actually vanishes, taking into account (2.4). This is because in our model the superpotential $\mathcal{W}$ is analytic in $\varphi$ by construction, while a non-zero $\varphi^{4}$ term in $\widetilde{\mathcal{L}}_{(4)}$ requires that the solution $\mathcal{W}$ of (2.4) contains $\log \varphi$ terms.
} 
or

$$
\begin{aligned}
\mathcal{A}_{\mathrm{sW}}[\Phi]=\frac{1}{\kappa^{2}}\left[\frac { 1 } { 4 ( d - 2 ) ^ { 2 } } \left(\frac{d}{d-1} R \widehat{\Gamma}^{k l}\right.\right. & \left.-2 R_{i}{ }^{k} \widehat{\Gamma}^{i l}+2 R_{i}{ }^{l} \widehat{\Gamma}^{i k}\right) \mathbb{D}_{k} \Psi_{+l}- \\
& \left.-\frac{i}{2} \partial_{i} \varphi \widehat{\Gamma}^{i} \zeta_{-}+\frac{1}{2(d-1)} \varphi^{2} \widehat{\Gamma}^{i j} \mathbb{D}_{i} \Psi_{+j}\right] .
\end{aligned}
$$

Notice that the terms in the first bracket

$$
\mathcal{A}_{\mathrm{sW}}^{(G)}\left[e, \Psi_{+}\right]=\frac{1}{\kappa^{2}} \frac{1}{4(d-2)^{2}}\left(\frac{d}{d-1} R \widehat{\Gamma}^{k l}-2 R_{i}{ }^{k} \widehat{\Gamma}^{i l}+2 R_{i}{ }^{l} \widehat{\Gamma}^{i k}\right) \mathbb{D}_{k} \Psi_{+l},
$$

are universal, in the sense that they do not depend on the model.

\subsubsection{Wess-Zumino consistency condition}

From the relation (4.35a) and corresponding equation for the toy model we find that the Weyl anomaly (5.3) and (5.5) satisfies the Wess-Zumino (WZ) consistency condition, which can be seen as follows. Defining the Weyl transformation operator $\delta_{\sigma}$ by

$$
\delta_{\sigma} \equiv \int_{\partial \mathcal{M}} d^{d} x \sum_{\Phi_{(0)}} \delta_{\sigma} \Phi_{(0)} \frac{\delta}{\delta \Phi_{(0)}}
$$

where $\Phi_{(0)}$ refers to the source for every field $\Phi$, the WZ consistency condition becomes $\left[\delta_{\sigma_{1}}, \delta_{\sigma_{2}}\right] S_{\text {ren }}=0$. This is equivalent to demanding that $\delta_{\sigma_{1}} \int d^{d} x \mathcal{A}_{\mathrm{W}} \sigma_{2}$ be symmetric in $\sigma_{1}$ and $\sigma_{2}$, which can be seen from (4.35a) since

$$
\sum_{\Phi_{(0)}} \delta_{\sigma_{1}} \Phi_{(0)} \frac{\delta}{\delta \Phi_{(0)}} \int d^{d} y \mathcal{A}_{\mathrm{W}} \sigma_{2}=\sigma_{1} \partial^{i}\left(T \partial_{i} \sigma_{2}\right)
$$

for a certain scalar function $T$. We note that the SUSY and super-Weyl invariance of the Weyl anomaly follows from $(4.35 \mathrm{~b})$ and $(4.35 \mathrm{c})$, which can be thought as the WZ consistency checks.

In order to see that the super-Weyl anomaly (5.8) satisfies the WZ consistency condition, first we need to find the algebra of relevant symmetries. From (B.38), one can readily see that $^{15}$

$$
\left[\delta_{\epsilon_{+}}, \delta_{\bar{\epsilon}_{-}^{\prime}}\right] e_{i}^{a}=\left(\delta_{\sigma}+\delta_{\lambda}\right) e_{i}^{a}, \quad\left[\delta_{\epsilon_{+}}, \delta_{\bar{\epsilon}_{-}^{\prime}}\right] \varphi^{I}=\left(\delta_{\sigma}+\delta_{\lambda}\right) \varphi^{I}
$$

with the parameters $\sigma=\frac{1}{2} \bar{\epsilon}_{-}^{\prime} \epsilon_{+}, \quad \lambda=\frac{1}{2} \bar{\epsilon}_{-}^{\prime} \Gamma^{a b} \epsilon_{+}$. Notice that in our analysis it is impossible to see the above commutator for the fermionic sources, since our consideration is limited to quadratic order in fermions. However, (5.12) provides the WZ consistency condition for the super-Weyl anomaly, namely

$$
\left.\left(\delta_{\epsilon_{+}} \int d^{d} x\left|\boldsymbol{e}_{(0)}\right| \bar{\epsilon}_{-}^{\prime} \mathcal{A}_{\mathrm{sW}}\left[\Phi_{(0)}\right]\right)\right|_{\text {bosonic }}=\left.\left(\left[\delta_{\epsilon_{+}}, \delta_{\bar{\epsilon}_{-}^{\prime}}\right] S_{\mathrm{ren}}\right)\right|_{\text {bosonic }}=-\int d^{d} x\left|\boldsymbol{e}_{(0)}\right| \sigma \mathcal{A}_{W}^{(B)}\left[\Phi_{(0)}\right]
$$

\footnotetext{
${ }^{15}$ Here the subscript $o$ is omitted again, which was used to denote the leading asymptotics of the variation parameters in appendix B.4.
} 
since $\delta_{\lambda} S_{\text {ren }}=0$. Here $\mathcal{A}_{W}^{(B)}$ refers to the bosonic sector of the Weyl anomaly. In the following we show (5.13) in detail, namely

$$
\begin{aligned}
\delta_{\epsilon_{+}} & \int d^{d} x \sqrt{-\gamma} \bar{\epsilon}_{-}^{\prime} \mathcal{A}_{\mathrm{sW}}= \\
= & \frac{1}{\kappa^{2}} \int d^{d} x \sqrt{-\gamma} \bar{\epsilon}_{-}^{\prime}\left[\frac{1}{4(d-2)^{2}}\left(\frac{d}{d-1} R \widehat{\Gamma}^{k l}-2 R_{i}{ }^{k} \widehat{\Gamma}^{i l}+2 R_{i}{ }^{l} \widehat{\Gamma}^{i k}\right) \mathbb{D}_{k} \mathbb{D}_{l} \epsilon_{+}\right. \\
& \left.-\frac{1}{4} \partial_{i} \varphi \widehat{\Gamma}^{i} \widehat{\Gamma}^{j} \partial_{j} \varphi \epsilon_{+}+\frac{1}{2(d-1)} \varphi^{2} \widehat{\Gamma}^{i j} \mathbb{D}_{i} \mathbb{D}_{j} \epsilon_{+}\right] \\
= & \frac{1}{\kappa^{2}} \int d^{d} x \sqrt{-\gamma} \bar{\epsilon}_{-}^{\prime}\left[\frac{1}{32(d-2)^{2}}\left(\frac{d}{d-1} R \widehat{\Gamma}^{k l}-2 R_{i}{ }^{k} \widehat{\Gamma}^{i l}+2 R_{i}{ }^{l} \widehat{\Gamma}^{i k}\right) R_{m n k l} \widehat{\Gamma}^{m n}\right. \\
& \left.-\frac{1}{4} \partial_{i} \varphi \partial^{i} \varphi+\frac{1}{16(d-1)} \varphi^{2} \widehat{\Gamma}^{i j} \widehat{\Gamma}^{k l} R_{i j k l}\right] \epsilon_{+} \\
= & \frac{1}{\kappa^{2}} \int d^{d} x \sqrt{-\gamma} \bar{\epsilon}_{-}^{\prime}\left[\frac{1}{32(d-2)^{2}}\left(-\frac{2 d}{d-1} R^{2}+8 R_{i j} R^{i j}\right)-\frac{1}{4} \partial_{i} \varphi \partial^{i} \varphi-\frac{1}{8(d-1)} \varphi^{2} R\right] \epsilon_{+} \\
= & -\int d^{d} x \sqrt{-\gamma} \sigma \mathcal{A}_{W}^{(B)},
\end{aligned}
$$

where again $\sigma=\frac{1}{2} \bar{\epsilon}_{-}^{\prime} \epsilon_{+}$. In the above computation we omitted the subscript (0) for simplicity. In the same spirit, one can find another WZ consistency condition for the super-Weyl anomaly from

$$
\left[\delta_{\epsilon_{-}}, \delta_{\epsilon_{-}^{\prime}}\right] e_{i}^{a}=\left[\delta_{\epsilon_{-}}, \delta_{\epsilon_{-}^{\prime}}\right] \varphi^{I}=0
$$

We therefore have

$$
\left.\left(\left[\delta_{\epsilon_{-}}, \delta_{\epsilon_{-}^{\prime}}\right] S_{\text {ren }}\right)\right|_{\text {bosonic }}=0
$$

which can be shown in the same way.

\subsection{SUSY transformation of operators}

Now that the Ward identities are completely determined, we can use (5.2) to derive the $\mathrm{gPBH}$ transformation of the renormalized canonical momenta, without using the FG expansions of the induced fields $[31,53,54]$. In order to describe the gPBH transformation of the induced fields and their renormalized canonical momenta in an integrated way, we introduce the concept of a generalized Poisson bracket, which is defined by (see e.g. (6.30) in [53])

$$
\begin{aligned}
\left\{A\left[\Phi_{(0)}, \Pi^{\Phi}\right], B\left[\Phi_{(0)}, \Pi^{\Phi}\right]\right\} \equiv \int_{\partial \mathcal{M}} d^{d} x \sum_{\Phi_{(0)}}\left(\frac{\delta A}{\delta \Phi_{(0)}} \frac{\delta B}{\delta \Pi^{\Phi}}-\frac{\delta B}{\delta \Phi_{(0)}} \frac{\delta A}{\delta \Pi^{\Phi}}\right) \\
=\int_{\partial \mathcal{M}} d^{d} x\left(\frac{\delta A}{\delta e_{(0) i}^{a}} \frac{\delta B}{\delta \Pi_{a}^{i}}-\frac{\delta B}{\delta e_{(0) i}^{a}} \frac{\delta A}{\delta \Pi_{a}^{i}}+\frac{\delta A}{\delta \varphi_{(0)}^{I}} \frac{\delta B}{\delta \Pi_{I}^{\varphi}}-\frac{\delta B}{\delta \varphi_{(0)}^{I}} \frac{\delta A}{\delta \Pi_{I}^{\varphi}}\right. \\
+A \frac{\overleftarrow{\delta}}{\delta \Psi_{(0)+i}} \frac{\vec{\delta}}{\delta \Pi_{\Psi}^{i}} B-B \frac{\overleftarrow{\delta}}{\delta \Psi_{(0)+i}} \frac{\vec{\delta}}{\delta \Pi_{\Psi}^{i}} A+A \frac{\overleftarrow{\delta}}{\delta \zeta_{(0)-}^{I}} \frac{\vec{\delta}}{\delta \Pi_{I}^{\zeta}} B-B \frac{\overleftarrow{\delta}}{\delta \zeta_{(0)-}^{I}} \frac{\vec{\delta}}{\delta \Pi_{I}^{\zeta}} A \\
\left.+B \frac{\overleftarrow{\delta}}{\delta \Pi_{\Psi}^{i}} \frac{\vec{\delta}}{\delta \bar{\Psi}_{(0)+i}} A-A \frac{\overleftrightarrow{\delta}}{\delta \Pi_{\Psi}^{i}} \frac{\vec{\delta}}{\delta \bar{\Psi}_{(0)+i}} B+B \frac{\overleftrightarrow{\delta}}{\delta \Pi_{I}^{\bar{\zeta}}} \frac{\vec{\delta}}{\delta \bar{\zeta}_{(0)-}^{I}} A-A \frac{\overleftarrow{\delta}}{\delta \Pi_{I}^{\bar{\zeta}}} \frac{\vec{\delta}}{\delta \bar{\zeta}_{(0)-}^{I}} B\right)
\end{aligned}
$$


where $A\left[\Phi_{(0)}, \Pi^{\Phi}\right]$ and $B\left[\Phi_{(0)}, \Pi^{\Phi}\right]$ are arbitrary functions on the phase space $\left(\Phi_{(0)}, \Pi^{\Phi}\right)$. The Ward identities (5.2) then allow us to define a constraint function on the phase space

$$
\begin{aligned}
\mathcal{C}\left[\xi, \sigma, \epsilon_{ \pm}, \lambda\right] \equiv & \int_{\partial \mathcal{M}} d^{d} x\left\{\xi _ { i } \left(e_{(0)}^{a i} D_{j} \Pi_{a}^{j}-\left(\partial^{i} \varphi_{(0)}^{I}\right) \Pi_{I}^{\varphi}-\left(\bar{\zeta}_{(0)-}^{I} \overleftarrow{\mathbb{D}}^{i}\right) \Pi_{I}^{\bar{\zeta}}-\Pi_{I}^{\zeta}\left(\mathbb{D}^{i} \zeta_{(0)-}^{I}\right)\right.\right. \\
& \left.-\Pi_{\Psi}^{j}\left(\mathbb{D}^{i} \Psi_{(0)+j}\right)-\left(\bar{\Psi}_{(0)+j} \overleftarrow{\mathbb{D}}^{i}\right) \Pi_{\bar{\Psi}}^{j}+D_{j}\left(\Pi_{\Psi}^{j} \Psi_{(0)+}^{i}+\bar{\Psi}_{(0)+}^{i} \Pi_{\bar{\Psi}}^{j}\right)\right) \\
& +\sigma\left[-e_{(0) i}^{a} \Pi_{a}^{i}-\mathcal{G}^{I J} \partial_{I} \mathcal{W} \Pi_{J}^{\varphi}-\frac{1}{2}\left(\bar{\Psi}_{(0)+i} \Pi_{\bar{\Psi}}^{i}+\text { h.c. }\right)\right. \\
& \left.+\left(\frac{1}{2} \delta_{I}^{J}-\partial_{I} \partial^{J} \mathcal{W}\right)\left(\bar{\zeta}_{(0)-}^{I} \Pi_{J}^{\bar{\zeta}}+\text { h.c. }\right)-\left|\boldsymbol{e}_{(0)}\right| \mathcal{A}_{\mathrm{W}}\left[\Phi_{(0)}\right]\right] \\
& +\bar{\epsilon}_{+}\left(-\frac{1}{2} \Gamma^{a} \Psi_{(0)+i} \Pi_{a}^{i}-\frac{i}{2} \zeta_{(0)-}^{I} \Pi_{I}^{\varphi}+\frac{i}{2} \not \partial \varphi_{(0)}^{I} \Pi_{I} \bar{\zeta}_{I}+\mathbb{D}_{i} \Pi_{\bar{\Psi}}^{i}\right) \\
& +\left(\frac{1}{2} \Pi_{a}^{i} \bar{\Psi}_{(0)+i} \Gamma^{a}+\frac{i}{2} \Pi_{I}^{\varphi} \bar{\zeta}_{(0)-}^{I}+\frac{i}{2} \Pi_{I}^{\zeta} \not \partial \varphi_{(0)}^{I}+\Pi_{\Psi}^{i} \overleftarrow{\mathbb{D}}_{i}\right) \epsilon_{+} \\
& +\bar{\epsilon}_{-}\left(i \mathcal{G}^{I J} \partial_{I} \mathcal{W} \Pi_{J}^{\bar{\zeta}}-\widehat{\Gamma}_{i} \Pi_{\Psi}^{i}+\left|\boldsymbol{e}_{(0)}\right| \mathcal{A}_{\mathrm{sW}}\left[\Phi_{(0)}\right]\right) \\
& +\left(\Pi_{\Psi}^{i} \widehat{\Gamma}_{i}-i \mathcal{G}^{I J} \partial_{I} \mathcal{W} \Pi_{J}^{\zeta}+\left|\boldsymbol{e}_{(0)}\right| \overline{\mathcal{A}}_{\mathrm{sW}}\left[\Phi_{(0)}\right]\right) \epsilon_{-} \\
& \left.-\lambda^{a b}\left[e_{(0)[a i} \Pi_{b]}^{i}+\frac{1}{4}\left(\bar{\zeta}_{(0)-}^{I} \Gamma_{a b} \Pi_{I}^{\bar{\zeta}}+\bar{\Psi}_{(0)+i} \Gamma_{a b} \Pi_{\bar{\Psi}}^{i}+\text { h.c. }\right)\right]\right\}
\end{aligned}
$$

which generates the gPBH transformation B.4 through the Poisson bracket ${ }^{16}$

$$
\begin{aligned}
& \delta_{\sigma, \epsilon_{ \pm}, \lambda} \Phi_{(0)}=\left\{\mathcal{C}\left[\sigma, \epsilon_{ \pm}, \lambda\right], \Phi_{(0)}\right\}, \quad \delta_{\sigma, \epsilon_{ \pm}, \lambda} \Pi^{\Phi}=\left\{\mathcal{C}\left[\sigma, \epsilon_{ \pm}, \lambda\right], \Pi^{\Phi}\right\}, \\
& \delta_{\xi}^{(\mathrm{cgct})} \Phi_{(0)}=\left\{\mathcal{C}[\xi], \Phi_{(0)}\right\}, \quad \delta_{\xi}^{(\mathrm{cgct})} \Pi^{\Phi}=\left\{\mathcal{C}[\xi], \Pi^{\Phi}\right\} .
\end{aligned}
$$

Here $\delta_{\xi}^{(\mathrm{cgct})}$ refers to the covariant general coordinate transformation (see e.g. section 11.3 of [46]), under which variation of the fields is given by

$$
\begin{array}{rlrl}
\delta_{\xi}^{(\mathrm{cgct})} e_{(0) i}^{a} & =D_{i} \xi^{a}, & \delta_{\xi}^{(\mathrm{cgct})} \varphi_{(0)}^{I} & =\xi^{a} \partial_{a} \varphi_{(0)}^{I} \equiv \xi^{i} \partial_{i} \varphi_{(0)}^{I}, \\
\delta_{\xi}^{(\mathrm{cgct})} \Psi_{(0)+i} & =\xi^{j} \mathbb{D}_{j} \Psi_{(0)+i}+\left(D_{i} \xi^{j}\right) \Psi_{(0)+j}, & \delta_{\xi}^{(\mathrm{cgct})} \zeta_{(0)-}^{I}=\xi^{a} \mathbb{D}_{a} \zeta_{(0)-}^{I} \equiv \xi^{i} \mathbb{D}_{i} \zeta_{(0)-}^{I},
\end{array}
$$

where $\xi^{a} \equiv \xi^{i} e_{(0) i}^{a}$. Meanwhile, $\delta_{\xi}$ given in B.4 is the general coordinate transformation and it is related to $\delta_{\xi}^{(\mathrm{cgct})}$ by

$$
\delta_{\xi}^{(\mathrm{cgct})}=\delta_{\xi}-\delta_{\lambda_{a b}=\omega_{j a b} \xi^{j}} .
$$

The reason why diffeomorphisms and local Lorentz transformations appear in a mixed way is that the constraint function and the Poisson bracket can only give a covariant quantity but $\delta_{\xi}$ in (B.38) is not covariant by itself. Moreover, SUSY transformations require the sources to be covariant and thus we are forced to see the covariant general coordinate transformation rather than the general coordinate transformation.

\footnotetext{
${ }^{16} \mathrm{It}$ is obvious that the gPBH transformation of the sources can be obtained through this Poisson bracket. In appendix D we show that the same holds for the canonical momenta.
} 
The useful variations of renormalized canonical momenta extracted from (5.19) are

$$
\begin{aligned}
& \delta_{\epsilon_{+}} \Pi_{\bar{\Psi}}^{i}=\frac{\delta}{\delta \bar{\Psi}_{(0)+i}} \mathcal{C}\left[\epsilon_{+}\right]=\frac{1}{2} \Pi_{a}^{i} \Gamma^{a} \epsilon_{+} \\
& \delta_{\epsilon_{-}} \Pi_{\bar{\Psi}}^{i}=\frac{\delta}{\delta \bar{\Psi}_{(0)+i}} \mathcal{C}\left[\epsilon_{-}\right]=\frac{\delta}{\delta \bar{\Psi}_{(0)+i}} \int d^{d} x\left|\boldsymbol{e}_{(0)}\right| \overline{\mathcal{A}}_{\mathrm{sW}}\left[\Phi_{(0)}\right] \epsilon_{-} \\
& =-\frac{\left|\boldsymbol{e}_{(0)}\right|}{\kappa^{2}} \frac{1}{8} \mathbb{D}_{k}\left(\left[\frac{2}{3} R_{(0)} \widehat{\Gamma}_{(0)}^{i k}-R_{(0) j}{ }^{k} \widehat{\Gamma}_{(0)}^{i j}+R_{(0) j}{ }^{i} \widehat{\Gamma}_{(0)}^{k j}\right] \epsilon_{-}\right)- \\
& -\frac{\left|\boldsymbol{e}_{(0)}\right|}{\kappa^{2}} \frac{1}{6} \widehat{\Gamma}^{i j} \mathbb{D}_{j}\left(\varphi_{(0)}^{2} \epsilon_{-}\right), \\
& \delta_{\epsilon_{+}} \Pi_{I}^{\bar{\zeta}}=\frac{\delta}{\delta \bar{\zeta}_{(0)-}^{I}} \mathcal{C}\left[\epsilon_{+}\right]=\frac{i}{2} \Pi_{I}^{\varphi} \epsilon_{+}, \\
& \delta_{\epsilon_{-}} \Pi_{I}^{\bar{\zeta}}=\frac{\delta}{\delta \bar{\zeta}_{(0)-}^{I}} \mathcal{C}\left[\epsilon_{-}\right]=\frac{\delta}{\delta \bar{\zeta}_{(0)-}^{I}} \int d^{d} x\left|\boldsymbol{e}_{(0)}\right| \overline{\mathcal{A}}_{\mathrm{sW}}\left[\Phi_{(0)}\right] \epsilon_{-}=\underline{-\frac{\left|\boldsymbol{e}_{(0)}\right|}{\kappa^{2}} \frac{i}{2} \partial_{i} \varphi_{(0)} \widehat{\Gamma}_{(0)}^{i} \epsilon_{-},} \\
& \delta_{\epsilon_{+}} \Pi_{I}^{\varphi}=\frac{\delta}{\delta \varphi_{(0)}^{I}} \mathcal{C}\left[\epsilon_{+}\right]=-\frac{i}{2} \partial_{i}\left(\Pi_{I}^{\zeta} \widehat{\Gamma}^{i} \epsilon_{+}\right), \\
& \delta_{\epsilon_{-}} \Pi_{I}^{\varphi}=\frac{\delta}{\delta \varphi_{(0)}^{I}} \mathcal{C}\left[\epsilon_{-}\right]=-i \partial_{I}\left(\mathcal{G}^{J K} \partial_{K} \mathcal{W}\right) \Pi_{J}^{\zeta} \epsilon_{-}+\frac{\delta}{\delta \varphi_{(0)}^{I}} \int d^{d} x\left|\boldsymbol{e}_{(0)}\right| \overline{\mathcal{A}}_{\mathrm{sW}}\left[\Phi_{(0)}\right] \epsilon_{-} \\
& =\underline{i \Pi^{\zeta} \epsilon_{-}+\frac{\left|\boldsymbol{e}_{(0)}\right|}{\kappa^{2}} \frac{1}{3} \varphi_{(0)} \bar{\Psi}_{(0)+j} \overleftarrow{\mathbb{D}}_{i} \widehat{\Gamma}_{(0)}^{j i} \epsilon_{-},}
\end{aligned}
$$

where $R_{(0)}, R_{(0) i}{ }^{j}$ and $\widehat{\Gamma}_{(0)}^{i}$ denote the Ricci scalar, Ricci tensor, the Gamma matrix and the determinant of the metric for the vielbein $e_{(0) i}^{a}$. Here the underlined terms are computed specifically for the toy model. Notice that due to the super-Weyl anomaly, the $\epsilon_{-}$variation of the renormalized canonical momenta contains bosonic anomalous terms, which have a similar origin as the Schwarzian derivative appearing in the conformal transformation of the energy-momentum tensor of $2 \mathrm{D}$ CFT.

\subsection{BPS relations}

A bulk (bosonic) BPS configuration, which is a bosonic solution of the classical SUGRA action as well as is invariant under bulk SUSY transformation with a certain parameter, corresponds to a supersymmetric vacuum state of the dual field theory. Since the vacuum expectation value (vev) of many observables is computed in SUSY field theories, it is necessary to pay special attention to the bulk BPS solutions. The existence of a bulk BPS configuration implies that there exists a boundary SUSY parameter, under the gPBH transformation with which the fermionic sources are invariant, namely ${ }^{17}$

$$
\begin{array}{r}
\delta_{\eta} \Psi_{(0)+i} \equiv \delta_{\eta_{+}} \Psi_{(0)+i}+\delta_{\eta_{-}} \Psi_{(0)+i}=\mathbb{D}_{i} \eta_{+}-\widehat{\Gamma}_{(0) i} \eta_{-}=0 \\
\delta_{\eta} \zeta_{(0)-}^{I}=-\frac{i}{2} \widehat{\Gamma}_{(0)}^{i} \partial_{i} \varphi_{(0)}^{I} \eta_{+}+i \mathcal{G}^{I J} \partial_{J} \mathcal{W} \eta_{-}=0
\end{array}
$$

\footnotetext{
${ }^{17}$ Here we do not discuss the integrability condition of (5.23). For a discussion of the geometry of (5.23a), which is also known as the twistor equation, see e.g. section 3.1 in [3].
} 
where the first equation is usually referred to as the conformal Killing spinor (CKS) condition. Actually, the rigid supersymmetry of the boundary field theory is found by solving $(5.23)[1,3,55] .^{18}$

Now we show that the $\eta$-variation of any renormalized canonical momentum vanishes on a BPS solution, i.e.

$$
\left.\left.\delta_{\eta} \Pi^{\Phi}\right|_{\mathrm{BPS}} \equiv \delta_{\eta_{+}} \Pi^{\Phi}\right|_{\mathrm{BPS}}+\left.\delta_{\eta_{-}} \Pi^{\Phi}\right|_{\mathrm{BPS}}=0, \quad \text { for any source } \Phi_{(0)},
$$

where for the fermionic operators we have from (5.22)

$$
\begin{aligned}
\delta_{\eta} \Pi_{\bar{\Psi}}^{i} & =\frac{1}{2} \Pi_{a}^{i} \Gamma^{a} \eta_{+}+\frac{\delta}{\bar{\Psi}_{(0)+i}} \int_{\Sigma_{r}} d^{d} x\left|\boldsymbol{e}_{(0)}\right| \overline{\mathcal{A}}_{\mathrm{sW}}\left[\Phi_{(0)}\right] \eta_{-} \\
\delta_{\eta} \Pi_{I}^{\bar{\zeta}} & =\frac{i}{2} \Pi_{I}^{\varphi} \eta_{+}+\frac{\delta}{\delta \bar{\zeta}_{(0)-}^{I}} \int_{\Sigma_{r}} d^{d} x\left|\boldsymbol{e}_{(0)}\right| \overline{\mathcal{A}}_{\mathrm{sW}}\left[\Phi_{(0)}\right] \eta_{-}
\end{aligned}
$$

This is the holographic version of the fact that the vev of any $Q$-exact operator vanishes on SUSY vacua. We only need to consider the variation of the fermionic canonical momenta, since the $\eta$-variation of the bosonic canonical momenta trivially vanishes on a bosonic solution. One can in principle see (5.24) by expanding the bulk BPS equations. But since we have the SUSY and super-Weyl Ward identities, the form of which is the same for all SCFTs, we take advantage of the Ward identities (5.2a) for $\eta_{+}$and (5.2b) for $\eta_{-}$.

Taking into account the CKS condition (5.23), we obtain from the Ward identities that

$$
\begin{aligned}
& 0=\int_{\partial \mathcal{M}} d^{d} x\left[\left(-\frac{1}{2} \bar{\Psi}_{(0)+i} \Gamma^{a} \Pi_{a}^{i}-\frac{i}{2} \bar{\zeta}_{(0)-}^{I} \Pi_{I}^{\varphi}-\frac{i}{2} \Pi_{I}^{\zeta} \not \partial \varphi_{(0)}^{I}-\Pi_{\Psi}^{i} \overleftarrow{\mathbb{D}}_{i}\right) \eta_{+}\right. \\
& \left.+\left(i \mathcal{G}^{I J} \partial_{I} \mathcal{W} \Pi_{J}^{\zeta}-\Pi_{\Psi}^{i} \widehat{\Gamma}_{(0) i}-\left|\boldsymbol{e}_{(0)}\right| \overline{\mathcal{A}}_{\mathrm{sW}}\left[\Phi_{(0)}\right]\right) \eta_{-}\right] \\
& =\int_{\partial \mathcal{M}} d^{d} x\left(-\frac{1}{2} \bar{\Psi}_{(0)+i} \Gamma^{a} \Pi_{a}^{i} \eta_{+}-\frac{i}{2} \Pi_{I}^{\varphi} \bar{\zeta}_{(0)-}^{I} \eta_{+}-\left|\boldsymbol{e}_{(0)}\right| \overline{\mathcal{A}}_{\mathrm{sW}}\left[\Phi_{(0)}\right] \eta_{-}\right) .
\end{aligned}
$$

We emphasize that because the Ward identities are valid for any background, (5.26) holds at least to linear order in fermions for any value of $\bar{\Psi}_{(0)+i}$ and $\bar{\zeta}_{(0)-}^{I}$ as long as the bosonic sources admit a CKS. There might be a correction at order of $O\left(\left(\Psi_{(0)+}\right)^{2},\left(\zeta_{(0)_{-}}\right)^{2}\right)$, though. Note that non-trivial dependence of bosonic momenta $\Pi_{a}^{i}$ and $\Pi_{I}^{\varphi}$ on the fermionic sources occurs from the quadratic order in fermions, i.e.

$$
\left.\frac{\delta}{\delta \bar{\Psi}_{(0)+i}} \Pi_{a}^{i}\right|_{\Psi_{(0)+i}=\zeta_{(0)-}^{I}=\cdots=0}=0
$$

and so on. Therefore, by taking the functional derivative of (5.26) with respect to the fermionic sources and evaluating on a (bosonic) supersymmetric background, we obtain

\footnotetext{
${ }^{18}$ More precisely, most of the rigid $\mathcal{N}=1$ SUSY field theories on curved backgrounds require a U(1) $R$-symmetry gauge field to be turned on. In this case, which is discussed in [40], the covariant derivative $\mathbb{D}_{i}$ in (5.23a) becomes $\mathbb{D}_{i}+i g A_{i}$, where $g$ is the $R$-charge of the corresponding field.
} 
the (bosonic) identities

$$
\begin{gathered}
\frac{1}{2} \Pi_{a}^{i} \Gamma^{a} \eta_{+}+\frac{\delta}{\bar{\Psi}_{(0)+i}} \int_{\Sigma_{r}} d^{d} x\left|\boldsymbol{e}_{(0)}\right| \overline{\mathcal{A}}_{\mathrm{sW}}\left[\Phi_{(0)}\right] \eta_{-}=0, \\
-\frac{i}{2} \Pi_{I}^{\varphi} \eta_{+}-\frac{\delta}{\delta \bar{\zeta}_{(0)-}^{I}} \int_{\Sigma_{r}} d^{d} x\left|\boldsymbol{e}_{(0)}\right| \overline{\mathcal{A}}_{\mathrm{sW}}\left[\Phi_{(0)}\right] \eta_{-}=0,
\end{gathered}
$$

where we used (3.17). Therefore, we find that on BPS backgrounds

$$
\delta_{\eta} \Pi_{\bar{\Psi}}^{i}=0, \quad \delta_{\eta} \Pi_{I}^{\bar{\zeta}}=0,
$$

which confirms our claim.

Note that from the field theory point of view (5.24) is quite natural, since supersymmetric vacua are annihilated by the preserved supercharge $Q$.

In order to convince ourselves, let us check (5.24) for the toy model. First, let us recall that in the toy model, $d=4$ and scaling dimension of $\varphi$ is 3. Then, (5.28)s become

$$
\begin{aligned}
0= & -\Gamma^{a} \eta_{+} \Pi_{a}^{i}+\frac{\left|\boldsymbol{e}_{(0)}\right|}{\kappa^{2}} \frac{1}{4(d-2)^{2}} \mathbb{D}_{k}\left[\left(\frac{d}{d-1} R_{(0)} \widehat{\Gamma}_{(0)}^{k i}-2 R_{(0) j}{ }^{k} \widehat{\Gamma}_{(0)}^{j i}+2 R_{(0) j}{ }^{i} \widehat{\Gamma}_{(0)}^{j k}\right) \eta_{-}\right] \\
& +\frac{\left|\boldsymbol{e}_{(0)}\right|}{\kappa^{2}} \frac{1}{2(d-1)} \varphi_{(0)}^{2} \widehat{\Gamma}_{(0)}^{i j} \mathbb{D}_{j} \eta_{-}, \\
0= & -\frac{i}{2} \eta_{+} \Pi^{\varphi}+\frac{\left|\boldsymbol{e}_{(0)}\right|}{\kappa^{2}} \frac{i}{2} \widehat{\Gamma}_{(0)}^{i} \eta_{-} \partial_{i} \varphi_{(0)} .
\end{aligned}
$$

By combining (5.31) with the conformal Killing spinor equation for the toy model

$$
\begin{aligned}
\mathbb{D}_{i} \eta_{+} & =\widehat{\Gamma}_{(0) i} \eta_{-}, \\
\frac{1}{2} \widehat{\Gamma}_{(0)}^{i} \partial_{i} \varphi_{(0)} \eta_{+}+\varphi_{(0)} \eta_{-} & =0,
\end{aligned}
$$

we get

$$
-\varphi_{(0)} \Pi^{\varphi}+\frac{\left|\boldsymbol{e}_{(0)}\right|}{2 \kappa^{2}} \partial_{i} \varphi_{(0)} \partial^{i} \varphi_{(0)}=0 .
$$

This formula can be verified in the toy model by using the bulk BPS equation.

From the bulk BPS equation for $\zeta$ with the bulk SUSY parameter $\widehat{\epsilon}$

$$
\delta_{\widehat{\epsilon}} \zeta=\left(\not \partial \varphi-\mathcal{W}^{\prime}\right) \widehat{\epsilon}=0, \quad \mathcal{W}^{\prime} \equiv \frac{d}{d \varphi} \mathcal{W}(\varphi),
$$

one can obtain

$$
\dot{\varphi}=-\sqrt{\left(\mathcal{W}^{\prime}\right)^{2}+\partial_{i} \varphi \partial^{i} \varphi}
$$

where we fix the sign from leading asymptotics of $\varphi$. It then follows from the definition of $\pi^{\varphi}$ that

$$
\pi^{\varphi}=-\frac{\sqrt{-\gamma}}{\kappa^{2}} \dot{\varphi}=\frac{\sqrt{-\gamma}}{\kappa^{2}} \sqrt{\left(-\mathcal{W}^{\prime}\right)^{2}+\partial_{i} \varphi \partial^{i} \varphi} .
$$

On the other hand, the full bosonic counterterms are given by

$$
S_{\mathrm{ct}}=\frac{1}{\kappa^{2}} \int d^{d} x \sqrt{-\gamma}\left[\mathcal{W}-\frac{1}{4} R-\frac{1}{2} \log e^{-2 r}\left(\frac{1}{6} \varphi^{2} R+\partial_{i} \varphi \partial^{i} \varphi+\cdots\right)\right],
$$


where the ellipses denote the terms which do not depend on $\varphi$. The counterterms for the canonical momenta $\pi_{\text {ct }}^{\varphi}$ are then given by

$$
\pi_{\mathrm{ct}}^{\varphi}=\frac{\delta}{\delta \varphi} S_{\mathrm{ct}}=\frac{\sqrt{-\gamma}}{\kappa^{2}}\left[-\left(-\mathcal{W}^{\prime}\right)-\frac{1}{2} \log e^{-2 r}\left(\frac{1}{3} \varphi R-2 \square \varphi\right)\right] .
$$

Furthermore, from the conformal Killing spinor condition (5.23), we obtain

$$
0=\left(\square_{(0)} \varphi_{(0)}-\frac{1}{6} \varphi_{(0)} R_{(0)}\right) \eta_{+},
$$

which implies that the logarithmically divergent terms in (5.38) actually do not contribute to the counterterms. Eventually, the renormalized canonical momentum $\Pi^{\varphi}$ becomes

$$
\Pi^{\varphi}=\frac{1}{\kappa^{2}} \lim _{r \rightarrow+\infty} e^{-3 r} \sqrt{-\gamma} \frac{\partial_{i} \varphi \partial^{i} \varphi}{\sqrt{\left(-\mathcal{W}^{\prime}\right)^{2}+\partial_{i} \varphi \partial^{i} \varphi}+\left(-\mathcal{W}^{\prime}\right)}=\frac{\left|\boldsymbol{e}_{(0)}\right|}{2 \kappa^{2}} \frac{\partial_{i} \varphi_{(0)} \partial^{i} \varphi_{(0)}}{\varphi_{(0)}}
$$

which confirms the result (5.33) as well as the anomalous SUSY variation of the renormalized canonical momenta (5.22).

\subsection{Conserved charges and supersymmetry algebra with anomaly correction}

We recall that given a Killing vector $\xi^{i}$ which satisfies the Killing condition ${ }^{19}$

$$
\begin{aligned}
\mathcal{L}_{\xi} g_{(0) i j} & =D_{(0) i} \xi_{j}+D_{(0) j} \xi_{i}=0, \\
\mathcal{L}_{\xi} \varphi_{(0)}^{I} & =\xi^{i} \partial_{i} \varphi_{(0)}^{I}=0, \\
\mathcal{L}_{\xi} \zeta_{(0)-}^{I} & =\xi^{i} \mathbb{D}_{(0) i} \zeta_{(0)-}^{I}+\frac{1}{4} D_{(0) i} \xi_{j} \widehat{\Gamma}_{(0)}^{i j} \zeta_{(0)-}^{I}=0, \\
\mathcal{L}_{\xi} \Psi_{(0)+j} & =\xi^{i} \mathbb{D}_{(0) i} \Psi_{(0)+j}+\left(D_{(0) j} \xi_{i}\right) \Psi_{(0)+}^{i}+\frac{1}{4} D_{(0) k} \xi_{l} \widehat{\Gamma}_{(0)}^{k l} \Psi_{(0)+j}=0,
\end{aligned}
$$

we obtain a conservation law by combining (5.2d) with (5.2e), namely

$$
D_{i}\left[e_{j}^{a} \xi^{j} \Pi_{a}^{i}+\xi^{j}\left(\Pi_{\Psi}^{i} \Psi_{+j}+\bar{\Psi}_{+j} \Pi_{\bar{\Psi}}^{i}\right)\right]=0 .
$$

Note that we use the Kosmann's definition for the spinorial Lie derivative (see e.g. [56] and (A.11) of $[2]^{20}$ ) and the Lie derivative is related to $\mathrm{gPBH}$ transformations by

$$
\mathcal{L}_{\xi}=\delta_{\xi}^{(\mathrm{cgct})}+\delta_{\lambda_{a b}=-e_{a}^{i} e_{b}^{j} D_{[i} \xi_{j]}} .
$$

We emphasize that (5.42) holds for any background that admits a Killing vector. The conservation law (5.42) allows us to define a conserved charge associated with $\xi^{i}$, namely $[57,58]$

$$
\mathcal{Q}[\xi] \equiv \int_{\partial \mathcal{M} \cap \mathcal{C}} d \sigma_{i}\left(e_{j}^{a} \Pi_{a}^{i}+\Pi_{\Psi}^{i} \Psi_{+j}+\bar{\Psi}_{+j} \Pi_{\Psi}^{i}\right) \xi^{j},
$$

\footnotetext{
${ }^{19} g_{(0) i j} \equiv e_{(0) i}^{a} e_{(0) a j}$ is the induced metric on the boundary $\partial \mathcal{M}$.

${ }^{20}$ In the literature, including [56], the spinoral Lie derivative is defined by $\mathcal{L}_{\xi} \zeta=\xi^{i} \mathbb{D}_{i} \zeta-\frac{1}{4} D_{i} \xi_{j} \widehat{\Gamma}^{i j} \zeta$. The sign of the last term is minus, since the Gamma matrices there satisfy a Grassman algebra in Euclidean signature, while here we use the Minkowskian signature.
} 
which is independent of the choice of Cauchy surface $\mathcal{C}$. Note that the conserved charge $Q[\xi]$ is related to the constraint function by

$$
\mathcal{Q}[\xi]=\mathcal{C}\left[\xi, \lambda_{a b}=-e_{a}^{i} e_{b}^{j} D_{[i} \xi_{j]}\right] .
$$

We also have the conservation laws

$$
D_{i}\left(\Pi_{\Psi}^{i} \eta_{+}\right)=D_{i}\left(\bar{\eta}_{+} \Pi_{\bar{\Psi}}^{i}\right)=0
$$

which follow from the SUSY and super-Weyl Ward identities (5.2a) and (5.2b) for the CKS parameters $\eta_{+}$and $\bar{\eta}_{+}$. Note that the conservation laws (5.46) hold only on bosonic backgrounds. This allows us to define the conserved supercharges

$$
Q^{s}\left[\eta_{+}\right] \equiv \int_{\partial \mathcal{M} \cap \mathcal{C}} d \sigma_{i} \Pi_{\Psi}^{i} \eta_{+}, \quad Q^{s}\left[\bar{\eta}_{+}\right] \equiv \int_{\partial \mathcal{M} \cap \mathcal{C}} d \sigma_{i} \bar{\eta}_{+} \Pi_{\Psi}^{i}
$$

On a bosonic background we can identify these conserved charges with the constraint functions, namely

$$
Q^{s}\left[\eta_{+}\right]=\mathcal{C}\left[\eta_{+}, \eta_{-}\right], \quad Q^{s}\left[\bar{\eta}_{+}\right]=\mathcal{C}\left[\bar{\eta}_{+}, \bar{\eta}_{-}\right] .
$$

It then follows from (5.22) that on a bosonic background we have

$$
\begin{gathered}
\left.\left\{Q^{s}\left[\eta_{+}\right], Q^{s}\left[\bar{\eta}_{+}\right]\right\}\right|_{\text {Bosonic }}=\left.\int_{\partial \mathcal{M} \cap \mathcal{C}} d \sigma_{i} \bar{\eta}_{+}\left\{\mathcal{C}\left[\eta_{+}, \eta_{-}\right], \Pi_{\Psi}^{i}\right\}\right|_{\text {Bosonic }} \\
=\int_{\partial \mathcal{M} \cap \mathcal{C}} d \sigma_{i}\left[\frac{1}{2} \Pi_{a}^{i} \bar{\eta}_{+} \Gamma^{a} \eta_{+}+\bar{\eta}_{+}\left(\frac{\delta}{\delta \bar{\Psi}_{(0)+i}} \int_{\partial \mathcal{M}} d^{d} x\left|\boldsymbol{e}_{(0)}\right| \overline{\mathcal{A}}_{\mathrm{SW}} \eta_{-}\right)\right]_{\text {Bosonic }}
\end{gathered}
$$

In the case where the conformal Killing vector ${ }^{21}$

$$
\mathcal{K}^{i} \equiv i \bar{\eta}_{+} \widehat{\Gamma}^{i} \eta_{+}
$$

becomes a Killing vector, we can see that on a bosonic background the above commutator becomes

$$
\left\{Q^{s}\left[\eta_{+}\right], Q^{s}\left[\bar{\eta}_{+}\right]\right\}=-\frac{i}{2} \mathcal{Q}[\mathcal{K}]+\int_{\partial \mathcal{M} \cap \mathcal{C}} d \sigma_{i} \bar{\eta}_{+}\left(\frac{\delta}{\delta \bar{\Psi}_{(0)+i}} \int_{\partial \mathcal{M}} d^{d} x\left|\boldsymbol{e}_{(0)}\right| \overline{\mathcal{A}}_{\mathrm{sW}} \eta_{-}\right) .
$$

Not surprisingly, the super-Weyl anomaly corrects the supersymmetry algebra, too.

We can obtain other commutators such as $\left\{\mathcal{Q}[\xi], Q^{s}\left[\eta_{+}\right]\right\}$. It is possible because $\mathcal{Q}[\xi]$ for the Killing vector $\xi^{i}$ is conserved for any background so that

$$
\begin{aligned}
\int_{\partial \mathcal{M} \cap \mathcal{C}} d \sigma_{i}\left\{\mathcal{Q}[\xi], \Pi_{\Psi}^{i}\right\} \eta_{+} & =\int_{\partial \mathcal{M} \cap \mathcal{C}} d \sigma_{k}\left\{\mathcal{C}\left[\xi, \lambda_{a b}=-e_{a}^{i} e_{b}^{j} D_{[i} \xi_{j]}\right], \Pi_{\Psi}^{k}\right\} \eta_{+} \\
& =\int_{\partial \mathcal{M} \cap \mathcal{C}} d \sigma_{i}\left[-\Pi_{\Psi}^{i} \mathcal{L}_{\xi} \eta_{+}+D_{j}\left[\left(\xi^{j} \Pi_{\Psi}^{i}-\xi^{i} \Pi_{\Psi}^{j}\right) \eta_{+}\right]+\xi^{i} D_{j}\left(\Pi_{\Psi}^{j} \eta_{+}\right)\right],
\end{aligned}
$$

where the second term vanishes by using Stokes' theorem. The third term is also zero on a bosonic background, due to the conservation law. Therefore, we have

$$
\left\{\mathcal{Q}[\xi], Q^{s}\left[\eta_{+}\right]\right\}=-\int_{\partial \mathcal{M} \cap \mathcal{C}} d \sigma_{i} \Pi_{\Psi}^{i} \mathcal{L}_{\xi} \eta_{+}=-Q^{s}\left[\mathcal{L}_{\xi} \eta_{+}\right]
$$

\footnotetext{
${ }^{21}$ One can easily check that $\mathcal{K}^{i}$ satisfies the conformal Killing condition, by using (5.23).
} 
and in the same way

$$
\left\{\mathcal{Q}[\xi], Q^{s}\left[\bar{\eta}_{+}\right]\right\}=-\int_{\partial \mathcal{M} \cap \mathcal{C}} d \sigma_{i}\left(\bar{\eta}_{+} \overleftarrow{\mathcal{L}}_{\xi}\right) \Pi_{\bar{\Psi}}^{i}=-Q^{s}\left[\bar{\eta}_{+} \overleftarrow{\mathcal{L}}_{\xi}\right]
$$

since $\mathcal{L}_{\xi} \eta_{+}$and $\bar{\eta}_{+} \overleftarrow{\mathcal{L}}_{\xi}$ become conformal Killing spinors [56], i.e.

$$
\mathbb{D}_{i}\left(\mathcal{L}_{\xi} \eta_{+}\right)=\frac{1}{d} \widehat{\Gamma}_{i} \widehat{\Gamma}^{j} \mathbb{D}_{j}\left(\mathcal{L}_{\xi} \eta_{+}\right), \quad\left(\bar{\eta}_{+} \overleftarrow{\mathcal{L}}_{\xi}\right) \overleftarrow{\mathbb{D}}_{i}=\frac{1}{d}\left(\bar{\eta}_{+} \overleftarrow{\mathcal{L}}_{\xi}\right) \overleftarrow{\mathbb{D}}_{j} \widehat{\Gamma}^{j} \widehat{\Gamma}_{i}
$$

We note that (5.52) and (5.53) can be obtained in the other way, namely by computing

$$
\left\{Q^{s}\left[\eta_{+}\right], e_{j}^{a} \Pi_{a}^{i}+\Pi_{\Psi}^{i} \Psi_{+j}+\bar{\Psi}_{+j} \Pi_{\Psi}^{i}\right\}, \quad\left\{Q^{s}\left[\bar{\eta}_{+}\right], e_{j}^{a} \Pi_{a}^{i}+\Pi_{\Psi}^{i} \Psi_{+j}+\bar{\Psi}_{+j} \Pi_{\Psi}^{i}\right\} .
$$

In summary, the supersymmetry algebra on a curved (bosonic) background is

$$
\begin{aligned}
\left\{Q^{s}\left[\eta_{+}\right], Q^{s}\left[\bar{\eta}_{+}\right]\right\} & =-\frac{i}{2} \mathcal{Q}[\mathcal{K}]+\int_{\partial \mathcal{M} \cap \mathcal{C}} d \sigma_{i} \bar{\eta}_{+}\left(\frac{\delta}{\delta \bar{\Psi}_{(0)+i}} \int_{\partial \mathcal{M}} d^{d} x\left|\boldsymbol{e}_{(0)}\right| \overline{\mathcal{A}}_{\mathrm{sW}} \eta_{-}\right) \\
\left\{\mathcal{Q}[\xi], Q^{s}\left[\eta_{+}\right]\right\} & =-Q^{s}\left[\mathcal{L}_{\xi} \eta_{+}\right] \\
\left\{\mathcal{Q}[\xi], Q^{s}\left[\bar{\eta}_{+}\right]\right\} & =-Q^{s}\left[\bar{\eta}_{+} \overleftarrow{\mathcal{L}}_{\xi}\right]
\end{aligned}
$$

(5.56) closely resembles the SUSY algebra presented in the literature (see e.g. $[1,59,60]$ ), except for the super-Weyl anomaly-effect term.

We comment that (5.56) can be obtained without using the Poisson bracket, but in an equivalent and rather simple way. Recall that a symmetry of the field theory leads to a conservation of the corresponding (anomalous) Noether current $J^{i}$ (with the anomaly $\mathcal{A}_{J}$ )

$$
D_{i} J^{i}=\mathcal{A}_{J}
$$

from which we derive the variation of any operator $\mathcal{O}$ under the symmetry transformation (see e.g. (2.3.7) in [9]), namely

$$
\delta \mathcal{O}(x)+\int_{\partial \mathcal{M}} d^{d} y\left[D_{i} J^{i}(y)-\mathcal{A}_{J}(y)\right] \mathcal{O}(x)=0,
$$

where the second term can be computed by differentiating the relevant Ward identities with the source dual to operator $\mathcal{O}(x)$. Now one can readily see that the commutator of charges becomes

$$
\left\{Q_{1}, Q_{2}\right\}=\int_{\partial \mathcal{M} \cap \mathcal{C}} d \sigma_{i}\left(\delta_{1} J_{2}^{i}\right)=-\int_{\partial \mathcal{M} \cap \mathcal{C}} d \sigma_{i}\left(\int_{\partial \mathcal{M}} d^{d} y\left[D_{j} J_{1}^{j}(y)-\mathcal{A}_{J}(y)\right] J_{2}^{i}\right),
$$

and this prescription gives the same result with (5.56). See e.g. appendix E for derivation of $\left\{\mathcal{Q}[\xi], Q^{s}\left[\eta_{+}\right]\right\}$.

Now that we know from the last section that the l.h.s. of (5.51) vanishes on BPS backgrounds, we can conclude that the conserved charge associated with $\mathcal{K}^{i}$ on BPS backgrounds is totally fixed to be a functional derivative of the fermionic anomaly, namely

$$
\left.\mathcal{Q}[\mathcal{K}]\right|_{\mathrm{BPS}}=-2 i \int_{\partial \mathcal{M} \cap \mathcal{C}} d \sigma_{i} \bar{\eta}_{+}\left\{\frac{\delta}{\delta \bar{\Psi}_{(0)+i}} \int_{\partial \mathcal{M}} d^{d} x\left|\boldsymbol{e}_{(0)}\right| \overline{\mathcal{A}}_{\mathrm{sW}} \eta_{-}\right\} .
$$


Depending on the theory, $\mathcal{K}^{i}$ can be a combination of other Killing vectors such as $\partial_{t}$ and $\partial_{\psi}$, where $\psi$ refers to an angular coordinate. If this is the case, (5.60) can be regarded as a relation of the conserved charges on the supersymmetric background, but accompanied with an anomalous contribution. A similar relation is found in [40], which explains the discrepancy of the BPS condition (see e.g. (C.16) of [45])

$$
\langle H\rangle+\langle J\rangle+\gamma\langle Q\rangle=0
$$

for pure $A d S_{5}$ is precisely due to the anomalous contribution coming from the fermionic anomalies.

\section{Neumann boundary conditions}

Most of the computations so far are for the plus sign choice of (3.3b) at the beginning of section 3. This plus sign is actually equivalent to imposing Dirichlet boundary conditions on the spin $1 / 2$ field $\zeta$. Independently from this choice, we could determine the leading asymptotics of the scalar field, as emphasized before. This allows us to use the result of appendix B.3 and B.4 to conclude that the minus sign choice can be supersymmetric only when mass of its scalar SUSY-partner field belongs to the window [61-63]

$$
-\left(\frac{d}{2}\right)^{2} \leq m^{2} \leq-\left(\frac{d}{2}\right)^{2}+1
$$

In this window (3.3b) is already finite, implying that the canonical momentum of $\zeta_{-}$is not renormalized. Since $\zeta_{+}$by itself becomes the renormalized canonical momentum, the change of the sign from plus to minus is in fact a Legendre transformation of the renormalized on-shell action $\widehat{S}_{\text {ren }}$, which is equivalent to imposing Neumann boundary conditions on $\zeta_{-}$[64]. We have seen that $\widehat{S}_{\text {ren }}$ in the case of the plus sign choice is $\left(\epsilon_{+}\right)$supersymmetric (Dirichlet boundary conditions for scalar the field were implicitly imposed). Therefore, in order to preserve SUSY, one can expect that the boundary conditions for the scalar field should also be changed from Dirichlet to Neumann by a Legendre transformation.

To see this, one has to prove that the total Legendre transformation action

$$
S_{L}=-\int_{\Sigma_{r}}\left(\widehat{\pi}^{\zeta} \zeta_{-}+\bar{\zeta}_{-} \widehat{\pi}^{\bar{\zeta}}+\varphi \widehat{\pi}^{\varphi}\right), \quad \widehat{\pi}^{\bar{\zeta}}=\frac{\sqrt{-\gamma}}{\kappa^{2}} \zeta_{+}
$$

is invariant under an $\epsilon_{+}$transformation. Note that the variation of $\Pi_{I}^{\bar{\zeta}}$ gives directly how gPBH transformations act on $\zeta_{+}$. We again consider only one scalar field, and it is straightforward to extend the result here to the case for several scalar fields. From (5.22), one can find that the action of $\epsilon_{+}$on $S_{L}$ gives

$$
\delta_{\epsilon_{+}} S_{L} \sim-\int_{\Sigma_{r}}\left(\frac{i}{2} \widehat{\pi}^{\varphi} \bar{\zeta}_{-} \epsilon_{+}-\frac{i}{2} \partial_{i} \varphi \bar{\epsilon}_{+} \widehat{\Gamma}^{i} \widehat{\pi}^{\bar{\zeta}}-\frac{i}{2} \widehat{\pi}^{\varphi} \bar{\zeta}_{-} \epsilon_{+}-\frac{i}{2} \varphi \partial_{i}\left(\bar{\epsilon}_{+} \widehat{\Gamma}^{i} \widehat{\pi}^{\varphi}\right)+\text { h.c. }\right)=0 .
$$

This confirms that the total action $S+S_{L}$ for the Neumann boundary condition is still invariant under an $\epsilon_{+}$transformation. 
When it comes to the $\epsilon_{-}$variation of $S_{L}$, one finds that all the momentum-related terms are canceled, as before. The anomalous terms in the $\epsilon_{-}$variation of the renormalized canonical momenta, however, are not canceled but contribute to the $\epsilon_{-}$anomaly of $S+S_{L}$, together with $\mathcal{A}_{\mathrm{sW}}$. Namely, we obtain for the toy model that

$$
\delta_{\bar{\epsilon}_{-}}\left(S+S_{L}\right) \sim \int_{\Sigma_{r}} d^{d} x \sqrt{-\gamma} \bar{\epsilon}_{-}\left(\mathcal{A}_{\mathrm{sW}}^{(G)}-\frac{1}{6 \kappa^{2}} \varphi^{2} \widehat{\Gamma}^{i j} \mathbb{D}_{i} \Psi_{+j}\right) \equiv \int_{\Sigma_{r}} d^{d} x \sqrt{-\gamma} \bar{\epsilon}_{-} \mathcal{A}_{\mathrm{sW}}^{N}
$$

where the super-Weyl anomaly for Neumann boundary conditions is

$$
\mathcal{A}_{\mathrm{sW}}^{N}=\mathcal{A}_{\mathrm{sW}}^{(G)}-\frac{1}{6 \kappa^{2}} \varphi^{2} \widehat{\Gamma}^{i j} \mathbb{D}_{i} \Psi_{+j}
$$

\section{Concluding remarks}

In this work we have considered a generic $\mathcal{N}=25 \mathrm{D}$ supergravity theory with its fermionic sector in the context of holographic renormalization, through which we have obtained a complete set of supersymmetric counterterms. We have also found that scalars and their superpartners should satisfy the same boundary conditions in order for the theory to be consistent with SUSY.

The Ward identities (5.2) and the anomalies lead to rather remarkable consequences. By means of them, we showed that the SUSY transformation of local operators and the SUSY algebra of a theory which has $\mathcal{N}=14 \mathrm{D}$ SCFT in curved space as a UV fixed point become anomalous at the quantum level, see (5.25) and (5.56). We comment that once the $R$-symmetry gauge field is turned on, the $R$-charge and the related terms appear on r.h.s. of the first line (5.56), see [40]. Note that the anomalous terms are non-vanishing in general on curved backgrounds, even where all anomalies vanish.

We emphasize that our whole analysis here crucially relies on the existence of a scalar superpotential $\mathcal{W}$, in terms of which the Lagrangian is expressed. If the theory does not possess any superpotential, one could introduce a local and approximate superpotential which is sufficient for reproducing all divergent terms of the scalar potential, as done in [65]. Now one can see that the approximate superpotential should meet more restrictive criteria for the supersymmetric holographic renormalization. To make this point clear, let us discuss the approximate superpotential suggested in [65], see (5.15) there. One can find from the BPS equations (3.20) and (3.25) and the algebraic equation (3.26) in [65] that the BPS solution's flow to leading order is

$$
\begin{aligned}
& \frac{d \psi}{d r} \sim-\psi, \\
& \frac{d \varphi}{d r} \sim-\left(2 \varphi+\sqrt{\frac{2}{3}} \psi^{2}\right), \\
& \frac{d \chi}{d r} \sim-2 \chi\left(1+\frac{\psi^{2}}{\sqrt{6} \varphi}\right),
\end{aligned}
$$

where the r.h.s. of the last equation is a non-analytic function of $\varphi$ around $\varphi=0$. Hence it is impossible to find a analytic and approximate superpotential consistent with the BPS 
flow equations, which means that we need a more generic $\mathcal{N}=2$ gauged SUGRA model to study [65]. Notice that this inconsistency of the approximate superpotential with the BPS flow equations implies that the superpotential suggested in [65] is not approximate for the fermionic sector of SUGRA.

As long as there exists a superpotential (or at least an approximate one for the whole sector of SUGRA), many of our results here can be extended straightforwardly to other dimensions. A direct application of the analysis of this paper to other dimensions is to obtain the 2D super-Virasoro algebra with a central extension. Let us explain this here schematically. The super-Weyl anomaly in 2D SCFT can be easily found by using the trick of section 5.1.3, namely that the SUSY variation of the super-Weyl anomaly is equal to the Weyl anomaly. Since the Weyl anomaly is $e_{i}^{a} \mathcal{T}_{a}^{i}=\frac{c}{24 \pi} R$, we see immediately that the super-Weyl anomaly in $2 \mathrm{D}$ is $\Gamma_{i} \mathcal{S}^{i} \sim i \frac{c}{24 \pi} \Gamma^{i j} \mathbb{D}_{i} \Psi_{j}$ up to a constant coefficient, depending on the convention. It follows that the anomalous variation of the super-current operator is

$$
\delta_{\eta} \mathcal{S}^{i}=-\frac{i}{4} \Gamma^{a} \eta \mathcal{T}_{a}^{i}-\frac{i c}{48 \pi} \widehat{\Gamma}^{i j} \widehat{\Gamma}^{k} \mathbb{D}_{j} \mathbb{D}_{k} \eta
$$

where $\eta$ is the $2 \mathrm{D}$ CKS, satisfying the condition

$$
\mathbb{D}_{i} \eta=\frac{1}{2} \widehat{\Gamma}_{i} \widehat{\Gamma}^{j} \mathbb{D}_{j} \eta, \quad \text { or } \quad \widehat{\Gamma}^{j} \widehat{\Gamma}^{i} \mathbb{D}_{j} \eta=0
$$

Note that the anomalous term in (7.2) vanishes only when the 2D Ricci scalar $R=0$ and $\eta$ is a spinor, all second derivatives of which vanish. Since (7.3) admits an infinite number of solutions, as 2D conformal Killing vector equation, one gets infinite number of conserved super-charges $G_{r}$, which are added to the Virasoro algebra to form the super-Virasoro algebra. Now one can see that the central extension in (see e.g. (10.2.11b) in [66])

$$
\left\{G_{r}, G_{s}\right\}=2 L_{r+s}+\frac{c}{12}\left(4 r^{2}-1\right) \delta_{r,-s}
$$

of the super-Virasoro algebra in 2D flat background is derived from the anomalous term of $(7.2)$.

One should keep in mind, however, that since the representation of the spinor fields strongly depends on the dimension of spacetime it might not be easy to put the SUGRA action into the form of (2.1) in other (especially odd) dimensions.

\section{Acknowledgments}

I would like to thank Jin U Kang and Ui Ri Mun for interesting discussions. I am grateful to Jin U Kang for a careful reading and important comments on the draft.

\section{A Notation, conventions for Gamma matrices and useful identities}

Throughout this paper Greek indexes $\mu, \nu$ and $\alpha, \beta, \cdots$ refer to the coordinate and flat directions in the bulk respectively, and the Latin indexes $i, j, m, n, p, q, \cdots$ and $a, b, \cdots$ refer to the coordinate and flat directions on the radial slice respectively. The flat indices 
which correspond to radial-like and time-like directions are special, so we denote them by $\bar{r}$ and $\bar{t}$ respectively. The capital Latin letters $A, B, \cdots$ indicate the coordinate directions on the scalar and hyperino manifold. $\nabla_{\mu}, D_{i}$ and $\mathbb{D}_{i}$ refer to the covariant derivative in the bulk and the covariant derivatives of the bosonic and fermionic fields on the radial slice respectively.

We use the hermitian representation of the Lorentzian Gamma matrices, following the convention in [46]. $\Gamma^{\alpha}$ and $\Gamma^{a}$ indicate the Gamma matrices along the flat directions in the bulk and the boundary, while $\Gamma^{\mu}$ and $\widehat{\Gamma}^{i}$ refer to the Gamma matrices along the coordinate directions in the bulk and the boundary. The relations between these Gamma matrices are provided in appendix C. Both in the bulk and on the boundary the hermitian conjugation of the Gamma matrix is given by

$$
\Gamma^{\mu \dagger}=\Gamma^{\bar{t}} \Gamma^{\mu} \Gamma^{\bar{t}}, \quad \widehat{\Gamma}^{i \dagger}=\Gamma^{\bar{t}} \widehat{\Gamma}^{i} \Gamma^{\bar{t}} .
$$

The following formulas, which hold in any $D$ dimensional spacetime (see e.g. section 3 in [46]), are frequently used in this paper.

$$
\begin{aligned}
\Gamma^{\mu \nu \rho} & =\frac{1}{2}\left\{\Gamma^{\mu}, \Gamma^{\nu \rho}\right\}, \\
\Gamma^{\mu \nu \rho \sigma} & =\frac{1}{2}\left[\Gamma^{\mu}, \Gamma^{\nu \rho \sigma}\right], \\
\Gamma^{\mu \nu \rho} \Gamma_{\sigma \tau} & =\Gamma^{\mu \nu \rho}{ }_{\sigma \tau}+6 \Gamma_{[\mu \nu}^{[\mu \nu} \delta_{\sigma]}^{\rho]}+6 \Gamma^{[\mu} \delta_{[\tau}^{\nu} \delta_{\sigma]}^{\rho]}, \\
\Gamma^{\mu \nu \rho \sigma} \Gamma_{\tau \lambda} & =\Gamma^{\mu \nu \rho \sigma}{ }_{\tau \lambda}+8 \Gamma^{[\mu \nu \rho}{ }_{[\lambda} \delta_{\tau]}^{\sigma]}+12 \Gamma^{[\mu \nu} \delta^{\rho}{ }_{[\lambda} \delta^{\sigma]}{ }_{\tau]}, \\
\left.\Gamma_{\mu \nu}, \Gamma_{\rho \sigma}\right] & =2\left(g_{\nu \rho} \Gamma_{\mu \sigma}-g_{\mu \rho} \Gamma_{\nu \sigma}-g_{\nu \sigma} \Gamma_{\mu \rho}+g_{\mu \sigma} \Gamma_{\nu \rho}\right), \\
\Gamma^{\mu \nu \rho} \Gamma_{\rho} & =(D-2) \Gamma^{\mu \nu}, \\
\Gamma^{\mu \nu \rho} \Gamma_{\rho \sigma} & =(D-3) \Gamma_{\sigma}^{\mu \nu}+2(D-2) \Gamma^{[\mu} \delta_{\sigma]}^{\nu,}, \\
\Gamma^{\mu \nu} \nabla_{\mu} \nabla_{\nu} \zeta & =-\frac{1}{4} R \zeta, \\
\Gamma^{\mu \nu \rho} \nabla_{\nu} \nabla_{\rho} \zeta & =-\frac{1}{4}\left(R \Gamma^{\mu}-2 R_{\nu}{ }^{\mu} \Gamma^{\nu}\right) \zeta,
\end{aligned}
$$

where $\delta$ refers to the Kronecker delta.

There are left and right acting functional derivatives with respect to fermionic variable $\psi$, namely

$$
\frac{\vec{\delta}}{\delta \bar{\psi}}, \frac{\overleftarrow{\delta}}{\delta \psi}
$$

and in most cases the rightarrow symbol $\rightarrow$ is omitted. Here $\bar{\psi}$ denotes the Dirac adjoint of the spinor $\psi$, namely

$$
\bar{\psi} \equiv \psi^{\dagger}\left(i \Gamma^{\bar{t}}\right) .
$$

The affine connection $\Gamma_{\nu \rho}^{\mu}$ is related to the spin connection by (see e.g. (7.100) in [46])

$$
\Gamma_{\mu \nu}^{\rho}=E_{\alpha}^{\rho}\left(\partial_{\mu} E_{\nu}^{\alpha}+\omega_{\mu}^{\alpha}{ }_{\beta} E_{\nu}^{\beta}\right) .
$$

In this work we consider the supergravity theory in the second order formalism. This means that our theory is torsionless and thus the spin connection can bre expressed in terms of 
the vielbein as

$$
\omega_{\mu \alpha \beta}=E_{\nu \alpha} \partial_{\mu} E_{\beta}^{\nu}+\Gamma_{\mu \nu}^{\rho} E_{\rho \alpha} E_{\beta}^{\nu} .
$$

The variation of the torsionless spin connection is

$$
\delta \omega_{\mu \alpha \beta}=E_{[\alpha}^{\nu} D_{\mu} \delta E_{\beta] \nu}-E_{[\alpha}^{\nu} D_{\nu} \delta E_{\beta] \mu}+e_{\alpha}^{\rho} E_{\beta}^{\nu} E_{\gamma \mu} D_{[\nu} \delta E_{\rho]}^{\gamma} .
$$

which is useful for many of our computations. The covariant derivatives of the fermionic fields are given by

$$
\begin{aligned}
\nabla_{\mu} \Psi_{\nu} & =\partial_{\mu} \Psi_{\nu}+\frac{1}{4} \omega_{\mu \alpha \beta} \Gamma^{\alpha \beta} \Psi_{\nu}-\Gamma_{\mu \nu}^{\rho} \Psi_{\rho} \\
\nabla_{\mu} \zeta^{I} & =\partial_{\mu} \zeta^{I}+\frac{1}{4} \omega_{\mu \alpha \beta} \Gamma^{\alpha \beta} \zeta^{I}
\end{aligned}
$$

\section{B ADM decomposition and generalized PBH transformation}

A preliminary step of the Hamiltonian analysis of the gravitational theory is to decompose the variables of theory including the metric (or the vielbeins) into a radial-like (or time-like) direction and the other transverse directions (a.k.a. ADM decomposition [67]). Coupling gravity to spinor fields requires vielbeins to appear in the action explicitly and thus the ADM decomposition of the vielbeins instead of the metric should be done.

The ADM decomposition brings us a natural choice of gauge for the variables of the theory, which is referred to as the Fefferman-Graham (FG) gauge. In the FG gauge, the Hamiltonian analysis becomes much simpler.

\section{B.1 ADM decomposition of vielbein and the strong Fefferman-Graham gauge}

We begin with picking a suitable radial coordinate $r$ and doing the ADM decomposition of the metric to run the Hamiltonian formalism. Since the vielbein explicitly appears in the action through the covariant derivative of the spinor fields we need to decompose the vielbein itself rather than the metric.

Choosing the radial coordinate $r$, we describe the bulk space as a foliation of the constant $r$-slices, which we denote by $\Sigma_{r}$. Let $E^{\alpha}$ be the vielbeins of the bulk and we decompose them as

$$
E^{\alpha}=\left(N n^{\alpha}+N^{j} e_{j}^{\alpha}\right) d r+e_{j}^{\alpha} d x^{j}
$$

such that

$$
g_{\mu \nu}=E_{\mu}^{\alpha} E_{\nu}^{\beta} \eta_{\alpha \beta}, \quad \gamma_{i j}=e_{i}^{\alpha} e_{j}^{\beta} \eta_{\alpha \beta}, \quad n_{\alpha} e_{i}^{\alpha}=0, \quad \eta_{\alpha \beta} n^{\alpha} n^{\beta}=1,
$$

where $\alpha, \beta$ are bulk tangent space indices and $\eta=\operatorname{diag}(1,-1,1, \ldots, 1)$ (where $\eta_{\overline{t t}}=-1$ ). Note that $N$ and $N^{\alpha}$ are known as lapse and shift functions respectively. One can check that

$$
d s^{2} \equiv g_{\mu \nu} d x^{\mu} d x^{\nu}=\left(N^{2}+N^{i} N_{i}\right) d r^{2}+2 N_{i} d r d x^{i}+\gamma_{i j} d x^{i} d x^{j},
$$

which usually appears in textbooks. The inverse vielbeins are then given by

$$
E_{\alpha}^{r}=\frac{1}{N} n_{\alpha}, \quad E_{\alpha}^{i}=e_{\alpha}^{i}-\frac{N^{i}}{N} n_{\alpha} .
$$


It follows that

$$
\Gamma^{r}=\Gamma^{\alpha} E_{\alpha}^{r}=\frac{1}{N} n_{\alpha} \Gamma^{\alpha} \equiv \frac{1}{N} \Gamma .
$$

The extrinsic curvature on the radial slice $\Sigma_{r}$ is defined as

$$
K_{i j} \equiv \frac{1}{2 N}\left(\dot{\gamma}_{i j}-D_{i} N_{j}-D_{j} N_{i}\right)
$$

and $K \equiv \gamma^{i j} K_{i j}$. Moreover,

$$
\Gamma^{i}=\Gamma^{\alpha} E_{\alpha}^{i}=\widehat{\Gamma}^{i}-\frac{N^{i}}{N} \Gamma,
$$

where $\widehat{\Gamma}^{i} \equiv \Gamma^{\alpha} e_{\alpha}^{i}$. These vielbeins satisfy the relation

$$
e_{\alpha}^{i} e_{i}^{\beta}+n_{\alpha} n^{\beta}=\delta_{\alpha}^{\beta} .
$$

One can also see that the $\widehat{\Gamma}^{i}$ s satisfy the Clifford algebra on the slice and $\Gamma$ anticommutes with all $\widehat{\Gamma}^{i}$ s, i.e.

$$
\left\{\widehat{\Gamma}^{i}, \widehat{\Gamma}^{j}\right\}=2 \gamma^{i j}, \quad\left\{\widehat{\Gamma}^{i}, \Gamma\right\}=0 .
$$

It follows that the matrix $\Gamma$ can be used to define the 'radiality' (see e.g. [38]) on the slice, so that a generic spinor $\psi$ on the slice can be split into two by radiality, ${ }^{22}$

$$
\psi_{ \pm} \equiv \Gamma_{ \pm} \psi
$$

where $\Gamma_{ \pm} \equiv \frac{1}{2}(1 \pm \Gamma)$.

We recall that splitting spinor fields by their radiality is inevitable because different radiality leads to different asymptotic behavior [32, 33] as well as the constraints that relate the fermionic fields and their conjugate momenta should be solved in a Lorentz invariant way [36]. Remind that the fermionic fields that follow the first-derivative principle, differently from the bosonic fields that follow the second-derivative one, are related to their conjugate momenta by definition. Taking the Dirac Lagrangian as an example, we find that

$$
\mathcal{L}_{\text {Dirac }}=-\bar{\Psi} \Gamma^{\mu} \mathcal{D}_{\mu} \Psi-m \bar{\Psi} \Psi \Longrightarrow \Pi_{\Psi} \equiv \mathcal{L}_{\text {Dirac }} \frac{\overleftarrow{\delta}}{\delta \dot{\Psi}}=-\bar{\Psi} \Gamma^{r}
$$

In order to simplify the calculations that follow it is convenient to pick a particular vielbein frame so that

$$
n_{\alpha}=(1,0), \quad e_{\bar{r}}^{i}=0, \quad e_{i}^{\bar{r}}=0,
$$

and $e_{i}^{a}$ becomes the vielbein on the slice $\Sigma_{r}$. We will call the gauge (B.12) combined with the traditional Fefferman-Graham (FG) gauge

$$
N=1, \quad N^{i}=0, \quad \Psi_{r}=0,
$$

as the strong FG gauge. Namely, the strong FG gauge refers to

$$
E_{r}^{\bar{r}}=1, \quad E_{r}^{a}=0, \quad E_{i}^{\bar{r}}=0, \quad \Psi_{r}=0 .
$$

\footnotetext{
${ }^{22}$ When $d=D-1$ is even number, radiality can be regarded as chirality.
} 


\section{B.2 Decomposition of the covariant derivatives}

We obtain (see also (88) and (89) in [36])

$$
\begin{aligned}
& \omega_{r \alpha \beta}=n_{[\alpha} \dot{n}_{\beta]}+e_{i[\alpha} \dot{e}_{\beta]}{ }^{i}+2 n_{[\alpha} e_{\beta]}{ }^{i}\left(\partial_{i} N-N^{j} K_{j i}\right)-D_{i} N_{j} e_{[\alpha}{ }^{i} e_{\beta]}{ }^{j}, \\
& \omega_{i \alpha \beta}=n_{\alpha} \partial_{i} n_{\beta}+e_{j \alpha} \partial_{i} e_{\beta}{ }^{j}+\Gamma_{i j}^{k}[\gamma] e_{k \alpha} e_{\beta}{ }^{j}+2 K_{i}^{j} e_{j[\alpha} n_{\beta]},
\end{aligned}
$$

where we have used the Christoffel symbols

$$
\begin{aligned}
& \Gamma_{r r}^{r}=N^{-1}\left(\dot{N}+N^{i} \partial_{i} N-N^{i} N^{j} K_{i j}\right), \\
& \Gamma_{r i}^{r}=N^{-1}\left(\partial_{i} N-N^{j} K_{i j}\right), \\
& \Gamma_{i j}^{r}=-N^{-1} K_{i j}, \\
& \Gamma_{r r}^{i}=-N^{-1} N^{i} \dot{N}-N D^{i} N-N^{-1} N^{i} N^{j} \partial_{j} N+\dot{N}^{i}+N^{j} D_{j} N^{i}+2 N N^{j} K_{j}^{i}+N^{-1} N^{i} N^{k} N^{l} K_{k l}, \\
& \Gamma_{r j}^{i}=-N^{-1} N^{i} \partial_{j} N+D_{j} N^{i}+N^{-1} N^{i} N^{k} K_{k j}+N K_{j}^{i}, \\
& \Gamma_{i j}^{k}=\Gamma_{i j}^{k}[\gamma]+N^{-1} N^{k} K_{i j} .
\end{aligned}
$$

Denoting the spin connection on the radial cut-off as $\widehat{\omega}_{i a b}$, we get

$$
\begin{aligned}
\widehat{\omega}_{i a b} & =e_{j a} \partial_{i} e_{b}^{j}+\Gamma_{i j}^{k}[\gamma] e_{k a} e_{b}^{j}=\omega_{i a b}, \\
\omega_{i \alpha \beta} \Gamma^{\alpha \beta} & =\widehat{\omega}_{i a b} \Gamma^{a b}+2 K_{j i} e_{\alpha}^{j} n_{\beta} \Gamma^{\alpha \beta}=\widehat{\omega}_{i a b} \Gamma^{a b}+2 K_{j i} \widehat{\Gamma}^{j} \Gamma, \\
\omega_{r \alpha \beta} \Gamma^{\alpha \beta} & =e_{i a} \dot{e}_{b}^{i} \Gamma^{a b}+2 \Gamma \widehat{\Gamma}^{i}\left(\partial_{i} N-N^{j} K_{j i}\right)-\widehat{\Gamma}^{i j} D_{i} N_{j}, \\
\nabla_{i} \Psi_{j} & =\mathbb{D}_{i} \Psi_{j}+\frac{1}{2} K_{l i} \widehat{\Gamma}^{l} \Gamma \Psi_{j}+\frac{1}{N} K_{i j}\left(\Psi_{r}-N^{k} \Psi_{k}\right), \\
\nabla_{i} \Psi_{r} & =\mathbb{D}_{i} \Psi_{r}+\frac{1}{2} K_{j i} \widehat{\Gamma}^{j} \Gamma \Psi_{r}-\Gamma_{i r}^{j} \Psi_{j}-\Gamma_{i r}^{r} \Psi_{r}, \\
\nabla_{r} \Psi_{i} & =\dot{\Psi}_{i}+\frac{1}{4}\left[e_{a i} \dot{e}_{b}^{i} \Gamma^{a b}+2 \Gamma \widehat{\Gamma}^{j}\left(\partial_{j} N-N^{l} K_{l j}\right)-\widehat{\Gamma}^{j l} D_{j} N_{l}\right] \Psi_{i}-\Gamma_{i r}^{j} \Psi_{j}-\Gamma_{i r}^{r} \Psi_{r}, \\
\nabla_{i} \zeta & =\mathbb{D}_{i} \zeta+\frac{1}{2} K_{j i} \widehat{\Gamma}^{j} \Gamma \zeta, \\
\nabla_{r} \zeta & =\dot{\zeta}+\frac{1}{4}\left[e_{a i} \dot{e}_{b}^{i} \Gamma^{a b}+2 \Gamma \widehat{\Gamma}^{j}\left(\partial_{j} N-N^{l} K_{l j}\right)-\widehat{\Gamma}^{j l} D_{j} N_{l}\right] \zeta,
\end{aligned}
$$

where

$$
\begin{aligned}
\mathbb{D}_{i} \Psi_{j} & =\partial_{i} \Psi_{j}+\frac{1}{4} \widehat{\omega}_{i a b} \Gamma^{a b} \Psi_{j}-\Gamma_{i j}^{k}[\gamma] \Psi_{k}, \\
\mathbb{D}_{i} \Psi_{r} & =\partial_{i} \Psi_{r}+\frac{1}{4} \widehat{\omega}_{i a b} \Gamma^{a b} \Psi_{r}, \\
\mathbb{D}_{i} \zeta & =\partial_{i} \zeta+\frac{1}{4} \omega_{i a b} \Gamma^{a b} \zeta,
\end{aligned}
$$

are the covariant derivatives of the spinors on the slice $\Sigma_{r}$. Note that in the final computations we used the gauge (B.12).

\section{B.3 Equations of motion and leading asymptotics of fermionic fields}

In order to discuss with the transformation law of the induced fields, we first study the leading asymptotic behavior of the fields, which can be understood from equations of 
motion. For $\Psi_{\mu}$ and $\zeta^{I}$ they are respectively,

$$
\Gamma^{\mu \nu \rho} \nabla_{\nu} \Psi_{\rho}-\mathcal{W} \Gamma^{\mu \nu} \Psi_{\nu}-\frac{i}{2} \mathcal{G}_{I J}\left(\not \partial \varphi^{I}+\mathcal{G}^{I K} \partial_{K} \mathcal{W}\right) \Gamma^{\mu} \zeta^{J}=0
$$

and

$$
\mathcal{G}_{I J}\left(\delta_{K}^{J} \not \mathbf{D}+\Gamma_{K L}^{J}[\mathcal{G}] \not \partial \varphi^{L}\right) \zeta^{K}+\mathcal{M}_{I J}(\varphi) \zeta^{J}+\frac{i}{2} \mathcal{G}_{I J} \Gamma^{\mu}\left(\not \partial \varphi^{J}-\mathcal{G}^{J K} \partial_{K} \mathcal{W}\right) \Psi_{\mu}=0 .
$$

Extracting the relevant terms, we obtain in the gauge (B.13)

$$
\begin{aligned}
0 \sim- & \widehat{\Gamma}^{i j}\left(\dot{\Psi}_{+j}-\frac{1}{2} \Psi_{+j}\right)+\widehat{\Gamma}^{i j}\left(\dot{\Psi}_{-j}+\frac{2 d-3}{2} \Psi_{-j}\right)+\widehat{\Gamma}^{i j k} \mathbb{D}_{j}\left(\Psi_{+k}+\Psi_{-k}\right), \\
0 \sim \dot{\zeta}_{+} & +\left(\frac{d}{2}+M_{\zeta}\right) \zeta_{+}-\dot{\zeta}_{-}-\left(\frac{d}{2}-M_{\zeta}\right) \zeta_{-}+\widehat{\Gamma}^{i} \mathbb{D}_{i} \zeta_{+}-\widehat{\Gamma}^{i} \mathbb{D}_{i} \zeta_{-}+\frac{i}{2}(\dot{\varphi}+\mu \varphi) \widehat{\Gamma}^{i} \Psi_{+i} \\
& +\frac{i}{2} \widehat{\Gamma}^{i} \widehat{\Gamma}^{j} \partial_{j} \varphi \Psi_{+i},
\end{aligned}
$$

where we assume that there is only one scalar $\varphi$ and one spin- $1 / 2$ field $\zeta$ for simplicity, and $M_{\zeta}$ which is the mass of $\zeta$ and $\mu$ are respectively

$$
\mu=-\left.\partial_{\varphi} \partial_{\varphi} \mathcal{W}\right|_{\varphi=0}, \quad M_{\zeta}=\left.\mathcal{M}_{\varphi \varphi}\right|_{\varphi=0},
$$

under the assumption that the scalar manifold metric is canonically normalized. $\mu$ and $M_{\zeta}$ are related by

$$
M_{\zeta}=-\mu+\frac{d-1}{2} .
$$

When $d>2$, the leading asymptotics of $\Psi_{+i}$ and $\Psi_{-i}$ are

$$
\begin{aligned}
& \Psi_{+i}(r, x) \sim e^{\frac{r}{2}} \Psi_{(0)+i}(x), \\
& \Psi_{-i}(r, x) \sim-\frac{1}{2} e^{-\frac{1}{2} r}\left(\frac{d-2}{d-1} \widehat{\Gamma}^{(0)}{ }_{i} \widehat{\Gamma}^{(0) k l}-\widehat{\Gamma}^{(0)}{ }_{i} k l\right) \mathbb{D}_{k}^{(0)} \Psi_{(0)+l}(x),
\end{aligned}
$$

where we used $e_{i}^{a}(r, x) \sim e^{r} e_{(0) i}^{a}(x)$ in AlAdS geometry, and $\Gamma^{(0)_{i}}$ and $\mathbb{D}^{(0)}$ refer to the Gamma matrices and the covariant derivative with respect to $e_{(0) i}^{a}$.

We need to be more careful, regarding $\zeta$. First, we note that since we would like to turn on an arbitrary source for the scalar field, the leading asymptotics of $\varphi$ should always be $\varphi(r, x) \sim e^{-\mu r} \varphi_{(0)}(x)$ as can be seen from (4.8c). Therefore, the final two terms in (B.22) can be discarded from the argument. Now there are 3 cases to consider:

1. $M_{\zeta}>1 / 2\left(\right.$ or $\left.\mu<\frac{d}{2}-1\right)$

The leading asymptotics of $\zeta_{-}$and $\zeta_{+}$are respectively

$$
\begin{aligned}
& \zeta_{-}(r, x) \sim e^{-\left(\mu+\frac{1}{2}\right) r} \zeta_{(0)-}(x), \\
& \zeta_{+}(r, x) \sim-\frac{1}{\mu+\frac{3}{2}}\left(e^{-\left(\mu+\frac{3}{2}\right) r} \widehat{\Gamma}^{(0) i} \mathbb{D}_{i}^{(0)} \zeta_{(0)-}(x)-\frac{i}{2} \widehat{\Gamma}^{(0) i} \widehat{\Gamma}^{(0) j} \partial_{j} \varphi_{(0)}(x) \Psi_{(0)+i}(x)\right) .
\end{aligned}
$$


2. $M_{\zeta}<-1 / 2\left(\right.$ or $\left.\mu>\frac{d}{2}\right)$

Here the behavior of $\zeta_{-}$and $\zeta_{+}$is opposite to the first case, namely

$$
\begin{aligned}
& \zeta_{+}(r, x) \sim e^{-\left(d-\mu-\frac{1}{2}\right) r} \zeta_{(0)+}(x), \\
& \zeta_{-}(r, x) \sim \frac{1}{d-\mu+\frac{1}{2}} e^{-\left(d-\mu+\frac{1}{2}\right) r} \widehat{\Gamma}^{(0) i} \mathbb{D}_{i}^{(0)} \zeta_{(0)+}(x) .
\end{aligned}
$$

3. $1 / 2 \geq M_{\zeta} \geq-1 / 2\left(\right.$ or $\left.\frac{d}{2} \geq \mu \geq \frac{d}{2}-1\right)$

This case actually coincides with the double quantization window [61-63] of the scalar field. The leading asymptotics are

$$
\begin{aligned}
& \zeta_{-}(r, x) \sim e^{-\left(\mu+\frac{1}{2}\right) r} \zeta_{(0)-}(x), \\
& \zeta_{+}(r, x) \sim e^{-\left(d-\mu-\frac{1}{2}\right) r} \zeta_{(0)+}(x) .
\end{aligned}
$$

\section{B.4 Generalized PBH transformations}

Let us find the most general bulk symmetry transformations that preserve the strong FG gauge (B.14), which we refer to as the generalized Penrose-Brown-Henneaux (gPBH) transformations [68-70]. We can immediately see that the local symmetries of the bulk SUGRA action (2.1) are diffeomorphisms, local Lorentz and supersymmetry transformations. Their infinitesimal action on the bulk fields takes the form

$$
\begin{aligned}
\delta_{\xi, \lambda, \epsilon} E_{\mu}^{\alpha} & =\xi^{\nu} \partial_{\nu} E_{\mu}^{\alpha}+\left(\partial_{\mu} \xi^{\nu}\right) E_{\nu}^{\alpha}-\lambda^{\alpha}{ }_{\beta} E_{\mu}^{\beta}+\frac{1}{2}\left(\bar{\epsilon} \Gamma^{\alpha} \Psi_{\mu}-\bar{\Psi}_{\mu} \Gamma^{\alpha} \epsilon\right), \\
\delta_{\xi, \lambda, \epsilon} \Psi_{\mu} & =\xi^{\nu} \partial_{\nu} \Psi_{\mu}+\left(\partial_{\mu} \xi^{\nu}\right) \Psi_{\nu}-\frac{1}{4} \lambda^{\alpha \beta} \Gamma_{\alpha \beta} \Psi_{\mu}+\left(\nabla_{\mu}+\frac{1}{2(d-1)} \mathcal{W} \Gamma_{\mu}\right) \epsilon, \\
\delta_{\xi, \lambda, \epsilon} \varphi^{I} & =\xi^{\mu} \partial_{\mu} \varphi^{I}+\frac{i}{2}\left(\bar{\epsilon} \zeta^{I}-\bar{\zeta}^{I} \epsilon\right), \\
\delta_{\xi, \lambda, \epsilon} \zeta^{I} & =\xi^{\mu} \partial_{\mu} \zeta^{I}-\frac{1}{4} \lambda^{\alpha \beta} \Gamma_{\alpha \beta} \zeta^{I}-\frac{i}{2}\left(\not \partial \varphi^{I}-\mathcal{G}^{I J} \partial_{J} \mathcal{W}\right) \epsilon
\end{aligned}
$$

with parameters $\xi^{\mu}, \lambda^{\alpha \beta}\left(\lambda^{\alpha \beta}=-\lambda^{\beta \alpha}\right)$ and $\epsilon$ respectively. The condition that imposes the strong FG gauge is then

$$
\begin{aligned}
& 0=\dot{\xi}^{r} \\
& 0=\dot{\xi}^{i} e_{i}^{a}-\lambda^{a}{ }_{\bar{r}} \\
& 0=\partial_{i} \xi^{r}-\lambda^{\bar{r}}{ }_{a} e_{i}^{a}+\frac{1}{2}\left(\bar{\epsilon}_{-} \Psi_{+i}+\bar{\Psi}_{+i} \epsilon_{-}-\bar{\epsilon}_{+} \Psi_{-i}-\bar{\Psi}_{-i} \epsilon_{+}\right), \\
& 0=\dot{\epsilon}_{+}+\dot{\epsilon}_{-}+\dot{\xi}^{i}\left(\Psi_{+i}+\Psi_{-i}\right)+\frac{1}{4} e_{a i} \dot{e}_{b}^{i} \Gamma^{a b}\left(\epsilon_{+}+\epsilon_{-}\right)+\frac{1}{2(d-1)} \mathcal{W}\left(\epsilon_{+}-\epsilon_{-}\right),
\end{aligned}
$$


and its solution is

$$
\begin{aligned}
\xi^{r} & =\sigma(x), \\
\xi^{i}(r, x) & =\xi_{o}^{i}(x)-\int^{r} d r^{\prime} \gamma^{i j}\left(r^{\prime}, x\right)\left[\partial_{j} \sigma+\frac{1}{2}\left(\bar{\epsilon}_{-} \Psi_{+j}+\bar{\Psi}_{+j} \epsilon_{-}-\bar{\epsilon}_{+} \Psi_{-j}-\bar{\Psi}_{-j} \epsilon_{+}\right)\right], \\
\lambda^{\bar{r} a} & =e^{a i}\left[\partial_{i} \sigma+\frac{1}{2}\left(\bar{\epsilon}_{-} \Psi_{+i}+\bar{\Psi}_{+i} \epsilon_{-}-\bar{\epsilon}_{+} \Psi_{-i}-\bar{\Psi}_{-i} \epsilon_{+}\right)\right] \\
\lambda^{a}{ }_{b} & =\lambda_{o}{ }^{a}{ }_{b}(x)+\cdots \\
\epsilon_{+}(r, x) & =\exp \left[\frac{r}{2}+\int^{r} d r^{\prime}\left(-\frac{\mathcal{W}+(d-1)}{2(d-1)}+\gamma^{i j}\left(r^{\prime}, x\right) \partial_{j} \sigma-\frac{1}{4} e_{a i} \dot{e}_{b}^{i} \Gamma^{a b}+O\left(\Psi^{2}\right)\right)\right] \epsilon_{o+}(x), \\
\epsilon_{-}(r, x) & =\exp \left[-\frac{r}{2}+\int^{r} d r^{\prime}\left(\frac{\mathcal{W}+(d-1)}{2(d-1)}+\gamma^{i j}\left(r^{\prime}, x\right) \partial_{j} \sigma-\frac{1}{4} e_{a i} \dot{e}_{b}^{i} \Gamma^{a b}+O\left(\Psi^{2}\right)\right)\right] \epsilon_{o-}(x),
\end{aligned}
$$

where $\sigma(x), \xi_{o}^{i}(x), \lambda_{o}{ }^{a} b(x)$ and $\epsilon_{o \pm}(x)$ are 'integration constants' which depend only on the transverse coordinates. Taking into account the leading behavior of the vielbeins and the gravitino one can see that the integral terms are subleading in (B.35). It follows that the leading asymptotics of the generalized $\mathrm{PBH}$ transformations are parameterized by the arbitrary independent transverse functions

$$
\sigma(x), \quad \xi_{o}^{i}(x), \quad \lambda_{o}^{a} b(x), \quad \epsilon_{o \pm}(x)
$$

which in fact correspond to the local conformal, diffeomorphism, Lorentz, SUSY, and superWeyl transformations of the induced fields on the radial slice $\Sigma_{r}$ respectively, as we will see soon.

Extracting the leading terms in (B.33) and taking into account the asymptotic behavior of the induced fields, we obtain how the sources transform, namely (from now on and also in the main text we do not write the subscript $o$ )

$$
\begin{aligned}
\delta_{\xi, \lambda, \epsilon} e_{i}^{a} & \sim \xi^{j} \partial_{j} e_{i}^{a}+\partial_{i} \xi^{j} e_{j}^{a}+e_{i}^{a} \sigma-\lambda_{b}^{a} e_{i}^{b}+\frac{1}{2}\left(\bar{\epsilon}_{+} \Gamma^{a} \Psi_{+i}+\text { h.c. }\right), \\
\delta_{\xi, \lambda, \epsilon} \Psi_{+i} & \sim \frac{1}{2} \Psi_{+i} \sigma+\xi^{j} \partial_{j} \Psi_{+i}+\left(\partial_{i} \xi^{j}\right) \Psi_{+j}+\mathbb{D}_{i} \epsilon_{+}-\widehat{\Gamma}_{i} \epsilon_{-}-\frac{1}{4} \lambda^{a b} \Gamma_{a b} \Psi_{+i}, \\
\delta_{\xi, \lambda, \epsilon} \varphi^{I} & \sim-\mathcal{G}^{I J} \partial_{J} \mathcal{W} \sigma+\xi^{i} \partial_{i} \varphi^{I}+\frac{i}{2}\left(\bar{\epsilon}_{+} \zeta_{-}^{I}+\text { h.c. }\right)+\frac{i}{2}\left(\bar{\epsilon}_{-} \zeta_{+}^{I}+\text { h.c. }\right),
\end{aligned}
$$

where we do not write down the variation of $\Psi_{-i}$ since unlike $\Psi_{+i}$ its leading term (B.26) does not transform as a source so that it cannot be used as a generalized coordinate [34].

As for $\zeta^{I}$, we need a careful discussion, since its leading behavior changes according to its mass. In the first case where $M_{\zeta} \geq 1 / 2, \zeta_{+}^{I}$ cannot be treated as a source, like the case of gravitino $\Psi_{-i}$. We also find that in the second case where $M_{\zeta}^{I} \leq-1 / 2$ (B.37c) is not consistent with the leading behavior of $\varphi \sim e^{-\mu r}$ due to the term $\frac{i}{2}\left(\bar{\epsilon}_{-} \zeta_{+}^{I}+\right.$ h.c. $) \sim e^{-\left(d-\mu^{I}\right) r}$, which implies that $\zeta_{+}^{I}$ cannot be turned on as a source, in order for the theory to be supersymmetric. In the final case where $1 / 2>M_{\zeta}>-1 / 2$, both $\zeta_{+}^{I}$ and $\zeta_{-}^{I}$ can be used as 
sources. The transformation law in this case is discussed in section 6. In summary, what we obtain is

$$
\begin{aligned}
\delta_{\xi, \lambda, \epsilon} \epsilon_{i}^{a} & \sim \xi^{j} \partial_{j} e_{i}^{a}+\partial_{i} \xi^{j} e_{j}^{a}+e_{i}^{a} \sigma-\lambda_{b}^{a} e_{i}^{b}+\frac{1}{2}\left(\bar{\epsilon}_{+} \Gamma^{a} \Psi_{+i}+\text { h.c. }\right) \\
\delta_{\xi, \lambda, \epsilon} \Psi_{+i} & \sim \frac{1}{2} \Psi_{+i} \sigma+\xi^{j} \partial_{j} \Psi_{+i}+\left(\partial_{i} \xi^{j}\right) \Psi_{+j}+\mathbb{D}_{i} \epsilon_{+}-\widehat{\Gamma}_{i} \epsilon_{-}-\frac{1}{4} \lambda^{a b} \Gamma_{a b} \Psi_{+i}, \\
\delta_{\xi, \lambda, \epsilon} \varphi^{I} & \sim \mathcal{G}^{I J} \partial_{J} \mathcal{W} \sigma+\xi^{i} \partial_{i} \varphi^{I}+\frac{i}{2}\left(\bar{\epsilon}_{+} \zeta_{-}^{I}+\text { h.c. }\right) \\
\delta_{\xi, \lambda, \epsilon} \zeta_{-}^{I} & \sim-\left(\frac{d}{2} \delta_{K}^{I}-\mathcal{G}^{I J} \mathcal{M}_{J K}\right) \zeta_{-}^{K} \sigma+\xi^{i} \partial_{i} \zeta_{-}^{I}+i \mathcal{G}^{I J} \partial_{J} \mathcal{W} \epsilon_{-}-\frac{i}{2} \widehat{\Gamma}^{i} \partial_{i} \varphi^{I} \epsilon_{+}-\frac{1}{4} \lambda^{a b} \Gamma_{a b} \zeta_{-}^{I},
\end{aligned}
$$

where we inverted the mass of $\zeta_{-}^{I}$ into the (scalar) $\sigma$-manifold language.

\section{Decomposition of the action and the fermion boundary terms}

In this appendix we decompose the terms in the fermionic sector of the action (2.1).

\section{C.1 Decomposition of the kinetic action of the hyperino field}

The kinetic term for $\zeta^{I}$ in the action (2.1) is decomposed as

$$
\begin{aligned}
& \mathcal{G}_{I J}\left(\bar{\zeta}^{I} \Gamma^{\mu} \nabla_{\mu} \zeta^{J}-\left(\nabla_{\mu} \bar{\zeta}^{I}\right) \Gamma^{\mu} \zeta^{J}\right) \\
= & \mathcal{G}_{I J} \bar{\zeta}^{I}\left(\Gamma^{r} \nabla_{r} \zeta^{J}+\Gamma^{i} \nabla_{i} \zeta^{J}\right)-\mathcal{G}_{I J} \bar{\zeta}^{I} \overleftarrow{\nabla}_{r} \Gamma^{r} \zeta^{J} \zeta^{J}-\mathcal{G}_{I J} \bar{\zeta}^{I} \overleftarrow{\nabla}_{i} \Gamma^{i} \zeta^{J} \\
= & \mathcal{G}_{I J} \bar{\zeta}^{I}\left[\frac{1}{N} \dot{\zeta}^{J}+\frac{1}{4 N} \Gamma\left(e_{a i} \dot{e}_{b}^{i} \Gamma^{a b}+2 \Gamma \widehat{\Gamma}^{i}\left(\partial_{i} N-N^{j} K_{i j}\right)-\widehat{\Gamma}^{i j} D_{i} N_{j}\right) \zeta^{J}\right. \\
& \left.\quad\left(\widehat{\Gamma}^{i}-\frac{N^{i}}{N} \Gamma\right)\left(\mathbb{D}_{i} \zeta^{J}+\frac{1}{2} K_{i j} \widehat{\Gamma}^{j} \Gamma \zeta^{J}\right)\right] \\
& -\mathcal{G}_{I J}\left[\dot{\bar{\zeta}}^{I}-\frac{1}{4} \bar{\zeta}^{I}\left[e_{a i} \dot{e}_{b}^{i} \Gamma^{a b}+2 \Gamma \widehat{\Gamma}^{i}\left(\partial_{i} N-N^{j} K_{i j}\right)-\widehat{\Gamma}^{i j} D_{i} N_{j}\right]\right] \frac{1}{N} \Gamma \zeta^{J} \\
& -\mathcal{G}_{I J}\left(\bar{\zeta}^{I} \overleftarrow{\mathbb{D}}_{i}-\frac{1}{2} K_{i j} \bar{\zeta}^{I} \widehat{\Gamma}^{j} \Gamma\right)\left(\widehat{\Gamma}^{i}-\frac{N^{i}}{N} \Gamma\right) \zeta^{J} \\
= & \frac{1}{N} \mathcal{G}_{I J}\left(\bar{\zeta}_{-}^{I} \dot{\zeta}_{+}^{J}-\bar{\zeta}_{+}^{I} \dot{\zeta}_{-}^{J}-\dot{\bar{\zeta}}_{-}^{I} \zeta_{+}^{J}+\dot{\bar{\zeta}}_{+}^{I} \zeta_{-}^{J}\right)+\frac{1}{2 N} \mathcal{G}_{I J} e_{a i} \dot{e}_{b}^{i} \bar{\zeta}^{I} \Gamma \Gamma^{a b} \zeta^{J} \\
& -\frac{1}{2 N} D_{i} N_{j} \mathcal{G}_{I J} \bar{\zeta}^{I} \Gamma \widehat{\Gamma}^{i j} \zeta^{J}+\mathcal{G}_{I J}\left(\bar{\zeta}^{I} \not D \zeta^{J}-\bar{\zeta}^{I} \overleftarrow{D} \overleftarrow{D}^{J}\right) \\
& -\frac{N^{i}}{N} \mathcal{G}_{I J}\left(\bar{\zeta}^{I} \Gamma \mathbb{D}_{i} \zeta^{J}-\bar{\zeta}^{I} \overleftarrow{\mathbb{D}}_{i} \Gamma \zeta^{J}\right)
\end{aligned}
$$

where the terms in the first bracket can be recast into

$$
\begin{aligned}
& \mathcal{G}_{I J}\left(\bar{\zeta}_{-}^{I} \dot{\zeta}_{+}^{J}-\bar{\zeta}_{+}^{I} \dot{\zeta}_{-}^{J}-\dot{\bar{\zeta}}_{-}^{I} \zeta_{+}^{J}+\dot{\bar{\zeta}}_{+}^{I} \zeta_{-}^{J}\right)=\mathcal{G}_{I J} \partial_{r}\left(\bar{\zeta}_{-}^{I} \zeta_{+}^{J}+\bar{\zeta}_{+}^{I} \zeta_{-}^{J}\right)-2 \mathcal{G}_{I J} \bar{\zeta}_{+}^{I} \dot{\zeta}_{-}^{J}-2 \mathcal{G}_{I J} \dot{\bar{\zeta}}_{-}^{I} \zeta_{+}^{J} \\
= & \frac{1}{\sqrt{-\gamma}} \partial_{r}\left(\mathcal{G}_{I J} \sqrt{-\gamma} \bar{\zeta}^{I} \zeta^{J}\right)-\left(N K+D_{k} N^{k}\right) \mathcal{G}_{I J} \bar{\zeta}^{I} \zeta^{J} \\
& -\left(\dot{\varphi}^{K}-N^{i} \partial_{i} \varphi^{K}+N^{i} \partial_{i} \varphi^{K}\right) \partial_{K} \mathcal{G}_{I J} \bar{\zeta}^{I} \zeta^{J}-2 \mathcal{G}_{I J} \bar{\zeta}_{+}^{I} \dot{\zeta}_{-}^{J}-2 \mathcal{G}_{I J} \dot{\bar{\zeta}}_{-}^{I} \zeta_{+}^{J}
\end{aligned}
$$


Finally, the hyperino kinetic terms are decomposed into

$$
\begin{aligned}
& \mathcal{G}_{I J}\left(\bar{\zeta}^{I} \Gamma^{\mu} \nabla_{\mu} \zeta^{J}-\left(\nabla_{\mu} \bar{\zeta}^{I}\right) \Gamma^{\mu} \zeta^{J}\right) \\
= & \frac{1}{N \sqrt{-\gamma}} \partial_{r}\left(\sqrt{-\gamma} \mathcal{G}_{I J} \bar{\zeta}^{I} \zeta^{J}\right)-\frac{2}{N} \mathcal{G}_{I J}\left(\bar{\zeta}_{+}^{I} \dot{\zeta}_{-}^{J}+\dot{\bar{\zeta}}_{-}^{I} \zeta_{+}^{J}\right)-\left(K+\frac{1}{N} D_{k} N^{k}\right) \mathcal{G}_{I J} \bar{\zeta}^{I} \zeta^{J} \\
& +\frac{1}{2 N} \mathcal{G}_{I J} e_{a i} \dot{e}_{b}^{i} \bar{\zeta}^{I} \Gamma^{a b} \Gamma \zeta^{J}-\frac{1}{N}\left(\dot{\varphi}^{K}-N^{i} \partial_{i} \varphi^{K}+N^{i} \partial_{i} \varphi^{K}\right) \partial_{K} \mathcal{G}_{I J} \bar{\zeta}^{I} \zeta^{J} \\
& +\mathcal{G}_{I J}\left(\bar{\zeta}^{I} \widehat{\Gamma}^{i} \mathbb{D}_{i} \zeta^{J}-\bar{\zeta}^{I} \overleftarrow{\mathbb{D}}_{i} \widehat{\Gamma}^{i} \zeta^{J}\right) \\
& +\frac{1}{N} \mathcal{G}_{I J}\left[-\frac{1}{2} D_{i} N_{j}\left(\bar{\zeta}^{I} \widehat{\Gamma}^{i j} \Gamma \zeta^{J}\right)-N^{i} \bar{\zeta}^{I} \Gamma \mathbb{D}_{i} \zeta^{J}+N^{i}\left(\bar{\zeta}^{I} \overleftarrow{\mathbb{D}}_{i}\right) \Gamma \zeta^{J}\right]
\end{aligned}
$$

\section{C.2 Gravitino part}

Repeating the same computation for the kinetic terms for gravitino as before, we obtain

$$
\begin{aligned}
& \left(\bar{\Psi}_{\mu} \Gamma^{\mu \nu \rho} \nabla_{\nu} \Psi_{\rho}-\bar{\Psi}_{\mu} \overleftarrow{\nabla}_{\nu} \Gamma^{\mu \nu \rho} \Psi_{\rho}\right)+\frac{1}{(D-2)} \bar{\Psi}_{\mu} \Gamma^{\mu \nu \rho}\left(\mathcal{W} \Gamma_{\nu}\right) \Psi_{\rho} \\
= & \frac{1}{N \sqrt{-\gamma}} \partial_{r}\left(\sqrt{-\gamma} \bar{\Psi}_{i} \widehat{\Gamma}^{i j} \Psi_{j}\right)-\frac{2}{N}\left(\dot{\bar{\Psi}}_{+i} \widehat{\Gamma}^{i j} \Psi_{-j}+\bar{\Psi}_{-i} \widehat{\Gamma}^{i j} \dot{\Psi}_{+j}\right) \\
& -\left(K+\frac{1}{N} D_{k} N^{k}\right) \bar{\Psi}_{i} \widehat{\Gamma}^{i j} \Psi_{j}-\frac{1}{4 N} e_{a k} \dot{e}_{b}^{k} \bar{\Psi}_{i} \Gamma\left\{\widehat{\Gamma}^{i j}, \Gamma^{a b}\right\} \Psi_{j} \\
& +\frac{1}{2 N} K_{l k} \bar{\Psi}_{i}\left(N\left[\widehat{\Gamma}^{i k j}, \widehat{\Gamma}^{l}\right] \Gamma+N^{i}\left[\widehat{\Gamma}^{k j}, \widehat{\Gamma}^{l}\right]-N^{j}\left[\widehat{\Gamma}^{k i}, \widehat{\Gamma}^{l}\right]\right) \Psi_{j} \\
& +\frac{1}{2 N} K_{k i}\left(\bar{\Psi}_{j}\left[\widehat{\Gamma}^{i j}, \widehat{\Gamma}^{k}\right] \Psi_{r}-\bar{\Psi}_{r}\left[\widehat{\Gamma}^{i j}, \widehat{\Gamma}^{k}\right] \Psi_{j}\right) \\
& +\frac{1}{N}\left(\bar{\Psi}_{j} \overleftarrow{\mathbb{D}}_{i} \Gamma \widehat{\Gamma}^{i j} \Psi_{r}+\bar{\Psi}_{r} \Gamma \widehat{\Gamma}^{i j} \mathbb{D}_{i} \Psi_{j}-\bar{\Psi}_{j} \Gamma \widehat{\Gamma}^{i j} \mathbb{D}_{i} \Psi_{r}-\bar{\Psi}_{r} \overleftarrow{\mathbb{D}}_{i} \Gamma \widehat{\Gamma}^{i j} \Psi_{j}\right) \\
& -\frac{1}{N} \mathcal{W}\left(\bar{\Psi}_{r} \Gamma \widehat{\Gamma}^{i} \Psi_{i}+\bar{\Psi}_{i} \widehat{\Gamma}^{i} \Gamma \Psi_{r}\right)-\frac{1}{4 N} \bar{\Psi}_{i}\left(2 \partial_{k} N\left[\widehat{\Gamma}^{i j}, \widehat{\Gamma}^{k}\right]-\left(D_{k} N_{l}\right) \Gamma\left\{\widehat{\Gamma}^{i j}, \widehat{\Gamma}^{k l}\right\}\right) \Psi_{j} \\
& +\frac{1}{N} \bar{\Psi}_{j}\left(N \widehat{\Gamma}^{j i k}-N^{j} \Gamma \widehat{\Gamma}^{i k}-N^{i} \Gamma \widehat{\Gamma}^{k j}-N^{k} \Gamma \widehat{\Gamma}^{j i}\right) \mathbb{D}_{i} \Psi_{k} \\
& +\frac{1}{N} \bar{\Psi}_{k} \overleftarrow{\mathbb{D}}_{i}\left(N \widehat{\Gamma}^{j i k}-N^{j} \widehat{\Gamma}^{i k}-N^{i} \Gamma \widehat{\Gamma}^{k j}-N^{k} \Gamma \widehat{\Gamma}^{j i}\right) \Psi_{j} \\
& -\frac{1}{N} \mathcal{W} \bar{\Psi}_{i}\left(N \widehat{\Gamma}^{i j}-N^{i} \widehat{\Gamma}^{j}+N^{j} \Gamma \widehat{\Gamma}^{i}\right) \Psi_{j} .
\end{aligned}
$$

\section{C.3 Decomposition of the other terms}

For the other terms, we get

$$
\begin{aligned}
& i \mathcal{G}_{I J} \bar{\zeta}^{I} \Gamma^{\mu}\left(\not \partial \varphi^{J}-\mathcal{G}^{J K} \partial_{K} \mathcal{W}\right) \Psi_{\mu}-i \mathcal{G}_{I J} \bar{\Psi}_{\mu}\left(\not \partial \varphi^{I}+\mathcal{G}^{I K} \partial_{K} \mathcal{W}\right) \Gamma^{\mu} \zeta^{J} \\
= & \frac{i}{N} \mathcal{G}_{I J}\left\{\frac{1}{N}\left(\dot{\varphi}^{J}-N^{j} \partial_{j} \varphi^{J}\right)\left[\bar{\zeta}^{I}\left(\Psi_{r}-N^{i} \Psi_{i}+N \widehat{\Gamma}^{i} \Gamma \Psi_{i}\right)-\left(\bar{\Psi}_{r}-N^{i} \bar{\Psi}_{i}+N \bar{\Psi}_{i} \Gamma \widehat{\Gamma}^{i}\right) \zeta^{I}\right]\right. \\
& \left.+\partial_{i} \varphi^{J}\left[\bar{\zeta}^{I} \Gamma \widehat{\Gamma}^{i}\left(\Psi_{r}-N^{j} \Psi_{j}\right)-\left(\bar{\Psi}_{r}-N^{j} \bar{\Psi}_{j}\right) \widehat{\Gamma}^{i} \Gamma \zeta^{I}\right]+N \partial_{i} \varphi^{I}\left(\bar{\zeta}^{I} \widehat{\Gamma}^{j} \widehat{\Gamma}^{i} \Psi_{j}-\bar{\Psi}_{j} \widehat{\Gamma}^{i} \widehat{\Gamma}^{j} \zeta^{I}\right)\right\} \\
- & \frac{i}{N} \partial_{I} \mathcal{W}\left[\bar{\zeta}^{I} \Gamma\left(\Psi_{r}-N^{i} \Psi_{i}\right)+\left(\bar{\Psi}_{r}-N^{i} \bar{\Psi}_{i}\right) \Gamma \zeta^{I}+N\left(\bar{\Psi}_{i} \widehat{\Gamma}^{i} \zeta^{I}+\zeta^{I} \widehat{\Gamma}^{i} \Psi_{i}\right)\right],
\end{aligned}
$$


and

$$
\begin{aligned}
& \mathcal{G}_{I J}\left[\bar{\zeta}^{I}\left(\Gamma_{K L}^{J} \not \partial \varphi^{L}\right) \zeta^{K}-\bar{\zeta}^{K}\left(\Gamma_{K L}^{J} \not \partial \varphi^{L}\right) \zeta^{I}\right] \\
= & \frac{1}{N} \partial_{K} \mathcal{G}_{I J}\left[\left(\dot{\varphi}^{J}-N^{i} \partial_{I} \varphi^{J}\right)\left(\bar{\zeta}^{I} \Gamma \zeta^{K}-\bar{\zeta}^{K} \Gamma \zeta^{I}\right)+N \partial_{i} \varphi^{J}\left(\bar{\zeta}^{I} \widehat{\Gamma}^{i} \zeta^{K}-\bar{\zeta}^{K} \widehat{\Gamma}^{i} \zeta^{I}\right)\right] .
\end{aligned}
$$

\section{Variation of the canonical momenta under the generalized PBH trans- formation}

By chain rule,

$$
\delta \widehat{S}_{\mathrm{ren}}=\int d^{d} x \sum_{\Phi} \Pi^{\Phi} \delta \Phi
$$

and let us define a symmetry transformation of $\widehat{S}_{\text {ren }}$ by

$$
\delta_{\xi}=\int d^{d} x \sum_{\Phi} \delta_{\xi} \Phi(x) \frac{\delta}{\delta \Phi(x)} .
$$

Let us also assume that this symmetry has an anomaly, i.e.

$$
\delta_{\xi} \widehat{S}_{\mathrm{ren}}=\int d^{d} x \sum_{\Phi} \Pi^{\Phi} \delta_{\xi} \Phi=\int d^{d} x\left|\boldsymbol{e}_{(0)}\right| \xi \mathcal{A}_{\xi} .
$$

Then, the definition of the constraint function $\mathcal{C}[\xi](5.18)$ can be written as

$$
\mathcal{C}[\xi]=-\int d^{d} x\left(\sum_{\Phi} \Pi^{\Phi} \delta_{\xi} \Phi-\left|\boldsymbol{e}_{(0)}\right| \xi \mathcal{A}_{\xi}\right) .
$$

Now we derive how the $\xi$-symmetry acts on $\Pi^{\Phi}$. It is

$$
\begin{aligned}
\delta_{\xi} \Pi^{\Phi}(x) & =\delta_{\xi} \frac{\delta}{\delta \Phi(x)} \widehat{S}_{\mathrm{ren}}=\left[\delta_{\xi}, \frac{\delta}{\delta \Phi(x)}\right] \widehat{S}_{\mathrm{ren}}+\frac{\delta}{\delta \Phi(x)} \delta_{\xi} \widehat{S}_{\mathrm{ren}} \\
& =-\int d^{d} y \sum_{\Phi^{\prime}}\left(\frac{\delta}{\delta \Phi(y)} \delta_{\xi} \Phi^{\prime}(x)\right) \Pi^{\Phi \prime}(x)+\frac{\delta}{\Phi(x)} \int d^{d} y\left|\boldsymbol{e}_{(0)}\right| \xi \mathcal{A}_{\xi} \\
& =-\frac{\delta}{\delta \Phi(x)} \int d^{d} y \sum_{\Phi^{\prime}}\left(\Pi^{\Phi \prime}(y) \delta_{\xi} \Phi^{\prime}(y)-\left|\boldsymbol{e}_{(0)}\right| \xi \mathcal{A}_{\xi}\right) \\
& =\left\{\mathcal{C}[\xi], \Pi^{\Phi}\right\}
\end{aligned}
$$

which confirms (5.19).

\section{E Derivation of the SUSY algebra without using Poisson bracket}

In this appendix we compute the anticommutator $\left\{\mathcal{Q}[\xi], Q^{s}\left[\eta_{+}\right]\right\}$. By differentiating the diffeomorphism Ward identity (5.2d) in the integral form with respect to $\Psi_{+k}(y)$, we get

$$
\begin{aligned}
0=\int_{\partial \mathcal{M}} d^{d} x \xi_{i} & {\left[e_{(0)}^{a i} D_{j} \Pi_{a}^{j}-\left(\partial^{i} \varphi_{(0)}^{I}\right) \Pi_{I}^{\varphi}-\left(\bar{\zeta}_{(0)-}^{I} \overleftarrow{\mathbb{D}}^{i}\right) \Pi_{I}^{\bar{\zeta}}-\Pi_{I}^{\zeta}\left(\mathbb{D}^{i} \zeta_{(0)-}^{I}\right)\right.} \\
& \left.-\Pi_{\Psi}^{j}\left(\mathbb{D}^{i} \Psi_{(0)+j}\right)-\left(\bar{\Psi}_{(0)+j} \overleftarrow{\mathbb{D}}^{i}\right) \Pi_{\Psi^{j}}^{j}+D_{j}\left(\Pi_{\Psi}^{j} \Psi_{(0)+}^{i}+\bar{\Psi}_{(0)+}^{i} \Pi_{\Psi}^{j}\right)\right]_{x} \Pi_{\Psi}^{k}(y) \\
& +\left(\xi^{i} \Pi_{\Psi}^{k}\right) \overleftarrow{\mathbb{D}}_{i}(y)-D_{j} \xi^{k} \Pi_{\Psi}^{j}(y)
\end{aligned}
$$


From the local Lorentz Ward identity (5.2e), we obtain

$$
0=\int_{\partial \mathcal{M}} d^{d} x \lambda^{a b}\left[e_{(0)[a i} \Pi_{b]}^{i}+\frac{1}{4}\left(\bar{\zeta}_{(0)-}^{I} \Gamma_{a b} \Pi_{I}^{\bar{\zeta}}+\bar{\Psi}_{(0)+i} \Gamma_{a b} \Pi_{\bar{\Psi}}^{i}+\text { h.c. }\right)\right]_{x} \Pi_{\Psi}^{k}(y)-\frac{1}{4} \lambda^{a b} \Pi_{\Psi}^{k} \Gamma_{a b}(y) .
$$

Summing these two expressions for the parameter $\lambda_{a b}=e_{a}^{i} e_{b}^{j} D_{[i} \xi_{j]}$, we obtain

$$
\begin{aligned}
0= & \int_{\partial \mathcal{M}} d^{d} x D_{j}\left[\xi^{i}\left(e^{a i} \Pi_{a}^{j}+\Pi_{\Psi}^{j} \Psi_{(0)+i}+\bar{\Psi}_{(0)+i} \Pi_{\Psi}^{j}\right)\right]_{x} \Pi_{\Psi}^{k}(y) \\
& +\left(\xi^{i} \Pi_{\Psi}^{k}\right) \overleftarrow{\mathbb{D}}_{i}(y)-D_{j} \xi^{k} \Pi_{\Psi}^{j}(y)-\frac{1}{4} D_{i} \xi_{j} \Pi_{\Psi}^{k} \widehat{\Gamma}^{i j}(y) .
\end{aligned}
$$

It follows from (5.59) that

$$
\begin{aligned}
\left\{\mathcal{Q}[\xi], Q^{s}\left[\eta_{+}\right]\right\} & =-\int_{\partial \mathcal{M} \cap \mathcal{C}} d \sigma_{k}(y) \int_{\partial \mathcal{M}} d^{d} x D_{j}\left[\xi^{i}\left(e^{a i} \Pi_{a}^{j}+\Pi_{\Psi}^{j} \Psi_{(0)+i}+\bar{\Psi}_{(0)+i} \Pi_{\Psi}^{j}\right)\right]_{x}\left(\Pi_{\Psi}^{k} \eta_{+}\right)_{y} \\
& =\int_{\partial \mathcal{M} \cap \mathcal{C}} d \sigma_{k}\left[\left(\xi^{i} \Pi_{\Psi}^{k}\right) \overleftarrow{\mathbb{D}}_{i}-D_{j} \xi^{k} \Pi_{\Psi}^{j}-\frac{1}{4} D_{i} \xi_{j} \Pi_{\Psi}^{k} \widehat{\Gamma}^{i j}\right] \eta_{+} \\
& =\int_{\partial \mathcal{M} \cap \mathcal{C}} d \sigma_{k}\left[D_{i}\left(\xi^{i} \Pi_{\Psi}^{k} \eta_{+}-\xi^{k} \Pi_{\Psi}^{i} \eta_{+}\right)+\xi^{k} D_{j}\left(\Pi_{\Psi}^{j} \eta_{+}\right)-\Pi_{\Psi}^{k} \mathcal{L}_{\xi} \eta_{+}\right] \\
& =-Q^{s}\left[\mathcal{L}_{\xi} \eta_{+}\right]
\end{aligned}
$$

where the first term in the third line is zero by Stokes' theorem and the second term vanishes due to the conservation law. One can confirm that the other commutators in (5.56) can be obtained in the same way.

Open Access. This article is distributed under the terms of the Creative Commons Attribution License (CC-BY 4.0), which permits any use, distribution and reproduction in any medium, provided the original author(s) and source are credited.

\section{References}

[1] G. Festuccia and N. Seiberg, Rigid Supersymmetric Theories in Curved Superspace, JHEP 06 (2011) 114 [arXiv: 1105.0689] [INSPIRE].

[2] T.T. Dumitrescu, G. Festuccia and N. Seiberg, Exploring Curved Superspace, JHEP 08 (2012) 141 [arXiv: 1205.1115] [INSPIRE].

[3] C. Klare, A. Tomasiello and A. Zaffaroni, Supersymmetry on Curved Spaces and Holography, JHEP 08 (2012) 061 [arXiv: 1205.1062] [INSPIRE].

[4] T.T. Dumitrescu, An introduction to supersymmetric field theories in curved space, J. Phys. A 50 (2017) 443005 [arXiv: 1608. 02957] [INSPIRE].

[5] N.A. Nekrasov, Seiberg-Witten prepotential from instanton counting, Adv. Theor. Math. Phys. 7 (2003) 831 [hep-th/0206161] [INSPIRE].

[6] V. Pestun, Localization of gauge theory on a four-sphere and supersymmetric Wilson loops, Commun. Math. Phys. 313 (2012) 71 [arXiv:0712.2824] [InSPIRE].

[7] H. Osborn, Weyl consistency conditions and a local renormalization group equation for general renormalizable field theories, Nucl. Phys. B 363 (1991) 486 [InSPIRE]. 
[8] I. Papadimitriou, Lectures on Holographic Renormalization, Springer Proc. Phys. 176 (2016) 131 [INSPIRE].

[9] J. Polchinski, String theory. Vol. 1: An introduction to the bosonic string, Cambridge University Press, (2007).

[10] J.M. Maldacena, The large-N limit of superconformal field theories and supergravity, Int. J. Theor. Phys. 38 (1999) 1113 [hep-th/9711200] [INSPIRE].

[11] S.S. Gubser, I.R. Klebanov and A.M. Polyakov, Gauge theory correlators from noncritical string theory, Phys. Lett. B 428 (1998) 105 [hep-th/9802109] [INSPIRE].

[12] E. Witten, Anti-de Sitter space and holography, Adv. Theor. Math. Phys. 2 (1998) 253 [hep-th/9802150] [INSPIRE].

[13] M. Henningson and K. Skenderis, The holographic Weyl anomaly, JHEP 07 (1998) 023 [hep-th/9806087] [INSPIRE].

[14] I. Bena, G. Giecold, M. Graña, N. Halmagyi and F. Orsi, Supersymmetric Consistent Truncations of IIB on $T^{1,1}$, JHEP 04 (2011) 021 [arXiv: 1008.0983] [INSPIRE].

[15] D. Cassani and A.F. Faedo, A supersymmetric consistent truncation for conifold solutions, Nucl. Phys. B 843 (2011) 455 [arXiv: 1008.0883] [inSPIRE].

[16] J.T. Liu and P. Szepietowski, Supersymmetry of consistent massive truncations of IIB supergravity, Phys. Rev. D 85 (2012) 126010 [arXiv:1103.0029] [InSPIRE].

[17] N. Halmagyi, J.T. Liu and P. Szepietowski, On $N=2$ Truncations of IIB on $T^{1,1}$, JHEP 07 (2012) 098 [arXiv:1111.6567] [INSPIRE].

[18] R. Argurio, M. Bertolini, D. Musso, F. Porri and D. Redigolo, Holographic Goldstino, Phys. Rev. D 91 (2015) 126016 [arXiv:1412.6499] [INSPIRE].

[19] M. Bertolini, D. Musso, I. Papadimitriou and H. Raj, A goldstino at the bottom of the cascade, JHEP 11 (2015) 184 [arXiv: 1509.03594] [INSPIRE].

[20] A. Ceresole, G. Dall'Agata, R. Kallosh and A. Van Proeyen, Hypermultiplets, domain walls and supersymmetric attractors, Phys. Rev. D 64 (2001) 104006 [hep-th/0104056] [InSPIRE].

[21] M. Günaydin and M. Zagermann, The gauging of five-dimensional, $N=2$ Maxwell-Einstein supergravity theories coupled to tensor multiplets, Nucl. Phys. B 572 (2000) 131 [hep-th/9912027] [INSPIRE].

[22] V. Balasubramanian and P. Kraus, A stress tensor for Anti-de Sitter gravity, Commun. Math. Phys. 208 (1999) 413 [hep-th/9902121] [INSPIRE].

[23] J. de Boer, E.P. Verlinde and H.L. Verlinde, On the holographic renormalization group, JHEP 08 (2000) 003 [hep-th/9912012] [INSPIRE].

[24] P. Kraus, F. Larsen and R. Siebelink, The gravitational action in asymptotically AdS and flat space-times, Nucl. Phys. B 563 (1999) 259 [hep-th/9906127] [INSPIRE].

[25] S. de Haro, S.N. Solodukhin and K. Skenderis, Holographic reconstruction of space-time and renormalization in the AdS/CFT correspondence, Commun. Math. Phys. 217 (2001) 595 [hep-th/0002230] [INSPIRE].

[26] M. Bianchi, D.Z. Freedman and K. Skenderis, How to go with an RG flow, JHEP 08 (2001) 041 [hep-th/0105276] [INSPIRE]. 
[27] M. Bianchi, D.Z. Freedman and K. Skenderis, Holographic renormalization, Nucl. Phys. B 631 (2002) 159 [hep-th/0112119] [INSPIRE].

[28] D. Martelli and W. Mueck, Holographic renormalization and Ward identities with the Hamilton-Jacobi method, Nucl. Phys. B 654 (2003) 248 [hep-th/0205061] [INSPIRE].

[29] K. Skenderis, Lecture notes on holographic renormalization, Class. Quant. Grav. 19 (2002) 5849 [hep-th/0209067] [INSPIRE].

[30] I. Papadimitriou and K. Skenderis, AdS/CFT correspondence and geometry, IRMA Lect. Math. Theor. Phys. 8 (2005) 73 [hep-th/0404176] [inSPIRE].

[31] I. Papadimitriou, Holographic renormalization as a canonical transformation, JHEP 11 (2010) 014 [arXiv: 1007.4592] [INSPIRE].

[32] M. Henningson and K. Sfetsos, Spinors and the AdS/CFT correspondence, Phys. Lett. B 431 (1998) 63 [hep-th/9803251] [INSPIRE].

[33] M. Henneaux, Boundary terms in the AdS/CFT correspondence for spinor fields, in Mathematical methods in modern theoretical physics. Proceedings, International Meeting, School and Workshop, ISPM'98, Tbilisi, Georgia, September 5-18, 1998, pp. 161-170, hep-th/9902137 [INSPIRE].

[34] G.E. Arutyunov and S.A. Frolov, On the origin of supergravity boundary terms in the AdS/CFT correspondence, Nucl. Phys. B 544 (1999) 576 [hep-th/9806216] [InSPIRE].

[35] A. Volovich, Rarita-Schwinger field in the AdS/CFT correspondence, JHEP 09 (1998) 022 [hep-th/9809009] [INSPIRE].

[36] J. Kalkkinen and D. Martelli, Holographic renormalization group with fermions and form fields, Nucl. Phys. B 596 (2001) 415 [hep-th/0007234] [InSPIRE].

[37] A.J. Amsel and G. Compere, Supergravity at the boundary of AdS supergravity, Phys. Rev. D 79 (2009) 085006 [arXiv: 0901.3609] [INSPIRE].

[38] D.Z. Freedman, K. Pilch, S.S. Pufu and N.P. Warner, Boundary Terms and Three-Point Functions: An AdS/CFT Puzzle Resolved, JHEP 06 (2017) 053 [arXiv:1611.01888] [INSPIRE].

[39] I. Papadimitriou, Holographic Renormalization of general dilaton-axion gravity, JHEP 08 (2011) 119 [arXiv:1106.4826] [InSPIRE].

[40] I. Papadimitriou, Supercurrent anomalies in 4d SCFTs, JHEP 07 (2017) 038 [arXiv: 1703.04299] [INSPIRE].

[41] L.F. Abbott, M.T. Grisaru and H.J. Schnitzer, A Supercurrent Anomaly in Supergravity, Phys. Lett. B 73 (1978) 71 [INSPIRE].

[42] M. Chaichian and W.F. Chen, The holographic supercurrent anomaly, Nucl. Phys. B 678 (2004) 317 [hep-th/0304238] [INSPIRE].

[43] A. Bilal, Lectures on Anomalies, arXiv:0802.0634 [INSPIRE].

[44] P. Benetti Genolini, D. Cassani, D. Martelli and J. Sparks, The holographic supersymmetric Casimir energy, Phys. Rev. D 95 (2017) 021902 [arXiv: 1606. 02724] [INSPIRE].

[45] P. Benetti Genolini, D. Cassani, D. Martelli and J. Sparks, Holographic renormalization and supersymmetry, JHEP 02 (2017) 132 [arXiv:1612.06761] [INSPIRE]. 
[46] D.Z. Freedman and A. Van Proeyen, Supergravity, Cambridge University Press, Cambridge, U.K., (2012).

[47] G.W. Gibbons and S.W. Hawking, Action Integrals and Partition Functions in Quantum Gravity, Phys. Rev. D 15 (1977) 2752 [InSPIRE].

[48] J. Lindgren, I. Papadimitriou, A. Taliotis and J. Vanhoof, Holographic Hall conductivities from dyonic backgrounds, JHEP 07 (2015) 094 [arXiv: 1505. 04131] [INSPIRE].

[49] I. Papadimitriou and K. Skenderis, Correlation functions in holographic RG flows, JHEP 10 (2004) 075 [hep-th/0407071] [INSPIRE].

[50] P. Di Francesco, P. Mathieu, and D. Senechal, Conformal Field Theory. Graduate Texts in Contemporary Physics. Springer-Verlag, New York, U.S.A. (1997).

[51] D. Anselmi, D.Z. Freedman, M.T. Grisaru and A.A. Johansen, Nonperturbative formulas for central functions of supersymmetric gauge theories, Nucl. Phys. B 526 (1998) 543 [hep-th/9708042] [INSPIRE].

[52] L. Bonora and S. Giaccari, Weyl transformations and trace anomalies in $N=1, D=4$ supergravities, JHEP 08 (2013) 116 [arXiv:1305.7116] [INSPIRE].

[53] M. Henneaux and C. Teitelboim, Quantization of gauge systems, Princeton University Press, Princeton, U.S.A., (1992).

[54] M. Cvetič and I. Papadimitriou, AdS $S_{2}$ holographic dictionary, JHEP 12 (2016) 008 [Erratum ibid. 01 (2017) 120] [arXiv:1608.07018] [INSPIRE].

[55] D. Cassani and D. Martelli, Supersymmetry on curved spaces and superconformal anomalies, JHEP 10 (2013) 025 [arXiv:1307.6567] [INSPIRE].

[56] J.M. Figueroa-O'Farrill, On the supersymmetries of Anti-de Sitter vacua, Class. Quant. Grav. 16 (1999) 2043 [hep-th/9902066] [INSPIRE].

[57] I. Papadimitriou and K. Skenderis, Thermodynamics of asymptotically locally AdS spacetimes, JHEP 08 (2005) 004 [hep-th/0505190] [INSPIRE].

[58] O.S. An, M. Cvetič and I. Papadimitriou, Black hole thermodynamics from a variational principle: Asymptotically conical backgrounds, JHEP 03 (2016) 086 [arXiv:1602.01508] [INSPIRE].

[59] D. Martelli and A. Passias, The gravity dual of supersymmetric gauge theories on a two-parameter deformed three-sphere, Nucl. Phys. B 877 (2013) 51 [arXiv:1306.3893] [INSPIRE].

[60] D. Cassani and D. Martelli, The gravity dual of supersymmetric gauge theories on a squashed $S^{1} \times S^{3}$, JHEP 08 (2014) 044 [arXiv: 1402.2278] [INSPIRE].

[61] P. Breitenlohner and D.Z. Freedman, Positive Energy in anti-de Sitter Backgrounds and Gauged Extended Supergravity, Phys. Lett. B 115 (1982) 197 [INSPIRE].

[62] V. Balasubramanian, P. Kraus and A.E. Lawrence, Bulk versus boundary dynamics in anti-de Sitter space-time, Phys. Rev. D 59 (1999) 046003 [hep-th/9805171] [INSPIRE].

[63] I.R. Klebanov and E. Witten, AdS/CFT correspondence and symmetry breaking, Nucl. Phys. B 556 (1999) 89 [hep-th/9905104] [INSPIRE].

[64] I. Papadimitriou, Multi-Trace Deformations in AdS/CFT: Exploring the Vacuum Structure of the Deformed CFT, JHEP 05 (2007) 075 [hep-th/0703152] [INSPIRE]. 
[65] N. Bobev, H. Elvang, D.Z. Freedman and S.S. Pufu, Holography for $N=2^{*}$ on $S^{4}$, JHEP 07 (2014) 001 [arXiv:1311.1508] [INSPIRE].

[66] J. Polchinski, String theory. Vol. 2: Superstring theory and beyond, Cambridge University Press, (2007).

[67] R.L. Arnowitt, S. Deser and C.W. Misner, Canonical variables for general relativity, Phys. Rev. 117 (1960) 1595 [INSPIRE].

[68] J.D. Brown and M. Henneaux, Central Charges in the Canonical Realization of Asymptotic Symmetries: An Example from Three-Dimensional Gravity, Commun. Math. Phys. 104 (1986) 207 [INSPIRE].

[69] R. Penrose and W. Rindler, Spinors and Space-Time, Cambridge Monographs on Mathematical Physics. Cambridge University Press, Cambridge, U.K., (2011).

[70] C. Imbimbo, A. Schwimmer, S. Theisen and S. Yankielowicz, Diffeomorphisms and holographic anomalies, Class. Quant. Grav. 17 (2000) 1129 [hep-th/9910267] [INSPIRE]. 